\title{
CHARACTERIZATION OF FLEXIBLE HYBRID ELECTRONICS USING STRETCHABLE SILVER INK AND ULTRA-THIN SILICON DIE
}

\author{
A Thesis \\ presented to \\ the Faculty of California Polytechnic State University, \\ San Luis Obispo
}

\author{
In Partial Fulfillment \\ of the Requirements for the Degree \\ Master of Science in Industrial Engineering
}

by

Joshua A. Ledgerwood

June 2017 
(C) 2017

Joshua A. Ledgerwood

ALL RIGHTS RESERVED 


\section{COMMITTEE MEMBERSHIP}

TITLE: Characterization of Flexible Hybrid Electronics Using Stretchable Silver Ink and Ultra-Thin Silicon Die

AUTHOR: Joshua A. Ledgerwood

DATE SUBMITTED: June 2017

COMMITTEE CHAIR: John Pan, Ph.D.

Professor of Industrial and Manufacturing Engineering

COMMITTEE MEMBER: Malcolm Keif, Ph.D.

Professor of Graphic Communications

COMMITTEE MEMBER: Xuan Wang, Ph.D.

Professor of Industrial and Manufacturing Engineering 


\begin{abstract}
Characterization of Flexible Hybrid Electronics Using Stretchable Silver Ink and UltraThin Silicon Die
\end{abstract}

Joshua A. Ledgerwood

Flexible Hybrid Electronics (FHEs) offer many advantages to the future of wearable technology. By combining the dynamic performance of conductive inks, and the functionality of ultra-thinned, traditional IC technology, new FHE devices allow for development of applications previously excluded by relying on a specific type of electronics technology.

The characterization and reliability analysis of stretchable conductive inks paired with ultra-thin silicon die in the $<50 \mu \mathrm{m}$ range was conducted. A silver based ink designed to be stretchable was screen printed on a TPU substrate and cured using box oven, conveyor convection oven, and photonic curing processes. Reliability tests were conducted including a tape test, crease test, wash test, and abrasion test. Optimization of each curing process resulted in all three methods' ability to achieve the ink sheet resistance specification of $<75 \mathrm{~m} \Omega / \mathrm{square} / 25 \mu \mathrm{m}$. Reliability tests on the printing concluded that, if fully cured, all samples achieve similar reliability performance. Additionally, a series of $10 \mathrm{~mm} \times 10 \mathrm{~mm}$ ultra-thin die were characterized using stylus profilometry and optical measurement in order to test the die quality and readiness for assembly. The die had been thinned from an initial thickness down of $600 \mu \mathrm{m}$ to a target of $50 \mu \mathrm{m}$. A direct inverse relationship was shown between die thickness and die warpage, likely due to high levels of internal stress caused by the dicing and thinning process. Finally, an innovate pairing of serpentine copper clad traces on TPU was tested for reliability performance using traditional solder for die attachment.

Keywords: printed electronics, flexible, hybrid, silicon, characterization, dicing, thinning 


\section{ACKNOWLEDGMENTS}

A great deal of thanks is owed to the many individuals who helped contribute to this overall project and assisted in the experimentation for this thesis. A special thanks to Wade Bedinger for helping conduct numerous reliability tests and data collection, to Wesley Powell (MATE) and Allison Tuuri (MATE) for their assistance with printing samples, and to Paul Swartz (ME), Maya Manzano (ME), and Julia Roche (ME) for development of a reliability testing device. A special thanks to David Otsu (IE) for assistance with operating and capturing SEM images.

I would also like to thank the faculty of Cal Poly for their dedication to my success both academically and professionally. I am grateful for my advisor Dr. Jianbiao Pan for providing me with the chance to work on this thesis topic and collaborate with the cutting-edge research into large scale manufacturability of flexible hybrid electronics. His continued support and persistence has led me to grow both academically and personally. My thanks to Dr. Malcolm Keif for his expertise in all things printed electronics and his consistently friendly demeanor. Additional thanks to Dr. Xuan Wang for his ability to provide guidance regardless of exterior circumstances. Also thanks to Dr. Xiaoying Rong for her expertise and assistance in the realm of printing and characterization. Lastly, I would like to thank Dr. Richard Savage and Dr. Jean Lee for their service as roles models in my personal, academic, and professional careers.

A multitude of thanks goes out to the Cal Poly Graduate Education office for their financial assistance through the Graduate Assistant research position. Likewise, an additional acknowledgment is due to the NextFlex organization whose financial and technical assistance has made this project possible.

Finally, I would like to thank the support from the department technicians David Laiho, Ladd Caine, and Robyn Burns who put forth the fullest efforts in maintaining lab equipment and keeping everything running.

This material is based upon work supported by Air Force Research Laboratory under agreement number FA8650-15-2-5401. The U.S. Government is authorized to reproduce and distribute reprints for Governmental purposes notwithstanding any copyright notation thereon. The views and conclusions contained herein are those of the authors and should not be interpreted as necessarily representing the official policies or endorsements, either expressed or implied, of Air Force Research Laboratory or the U.S. Government. 


\section{TABLE OF CONTENTS}

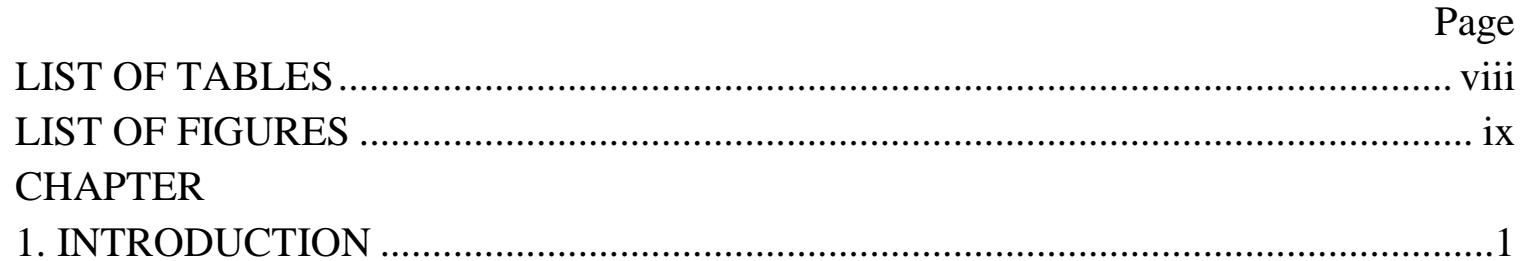

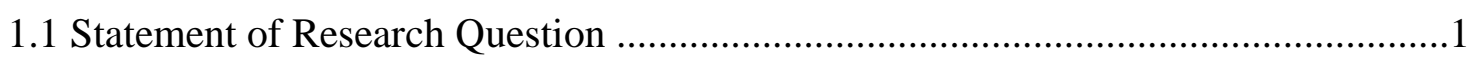

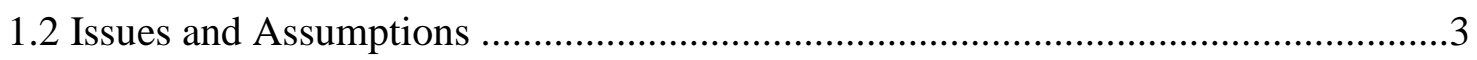

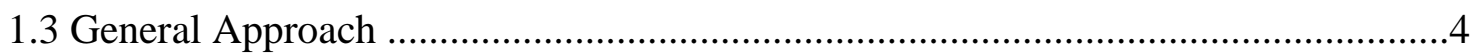

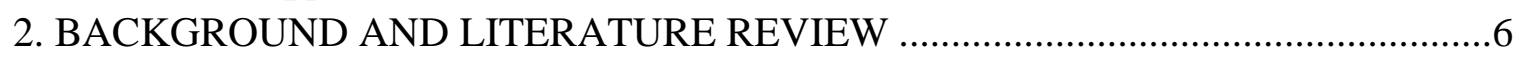

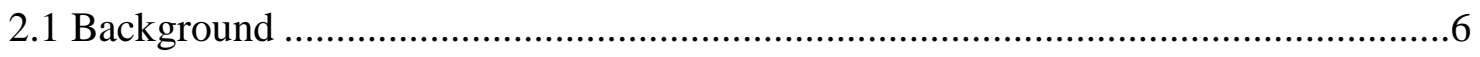

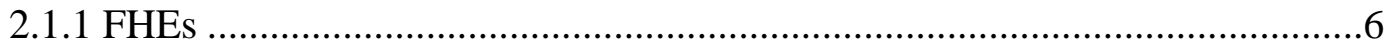

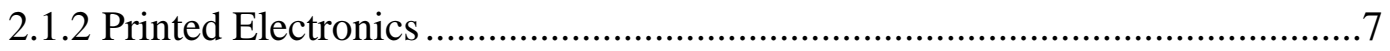

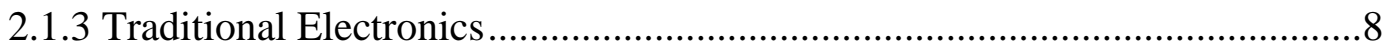

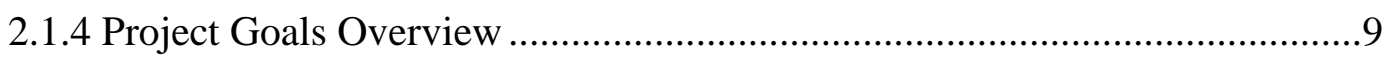

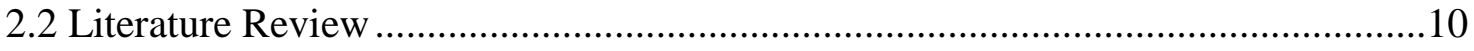

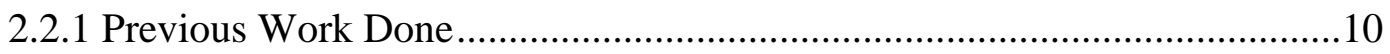

2.2.2 Printed Electronics Work ……………....................................................

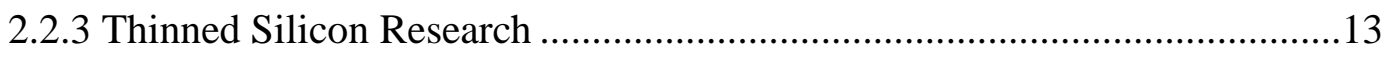

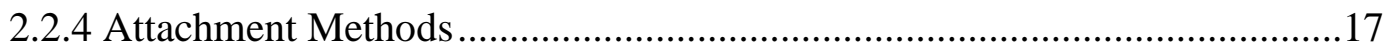

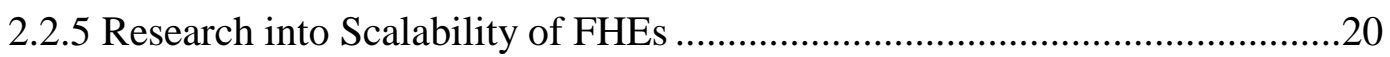

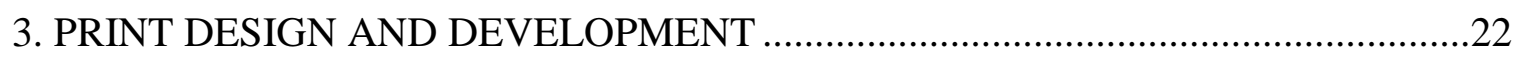

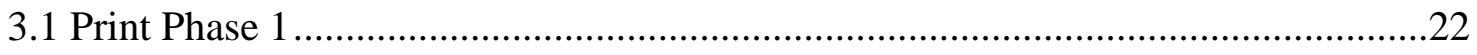

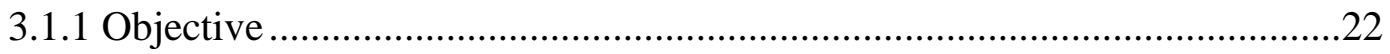

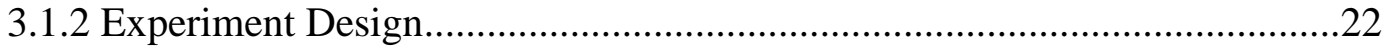

3.1.2.1 Equipment and Materials ..............................................................22

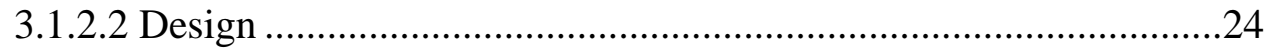

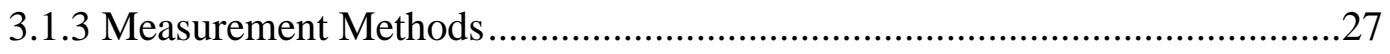

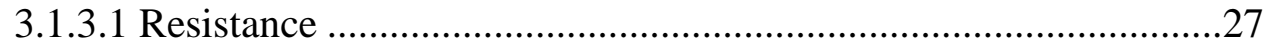

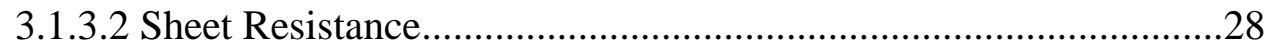

3.1.3.3 Trace Height................................................................................29

3.1.3.4 Trace Width and Spacing ............................................................30

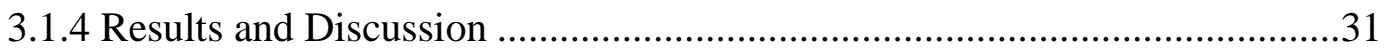

3.1.4.1 Photonic Curing ...........................................................................37

3.1.4.2 Preliminary Reliability Tests ........................................................38

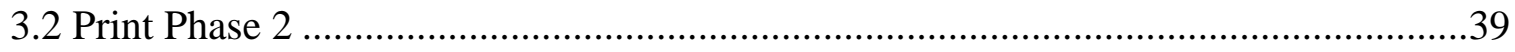

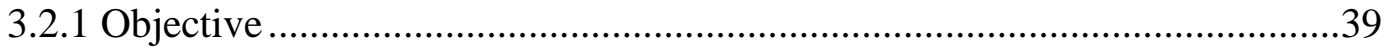

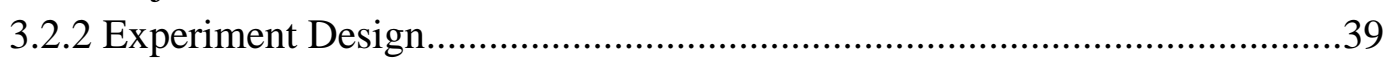

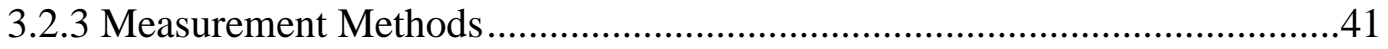

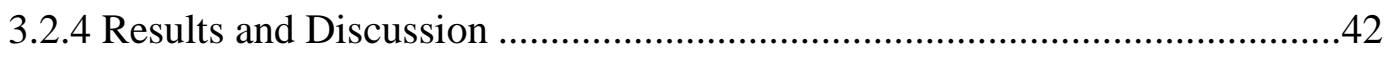

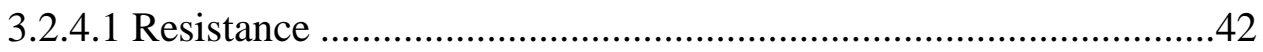


3.2.4.2 Reliability Tests: Crease Test ........................................................44

3.2.4.3 Reliability Tests: Tape Test ............................................................46

3.2.4.4 Reliability Tests: Wash Test ........................................................46

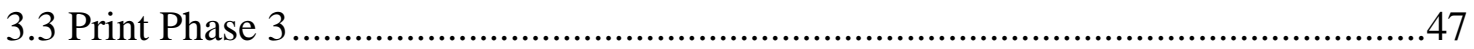

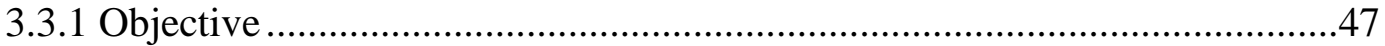

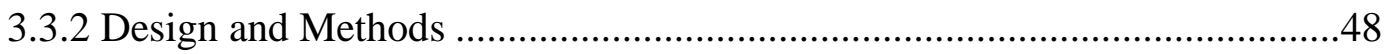

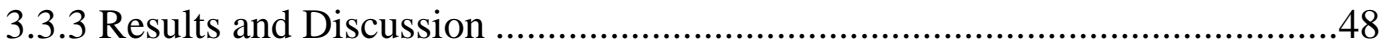

3.3.3.1 SEM Imaging of Printed Inks ....................................................51

3.4 Print Characterization Summary ………………............................................52

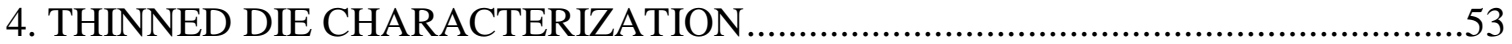

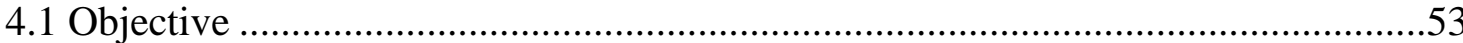

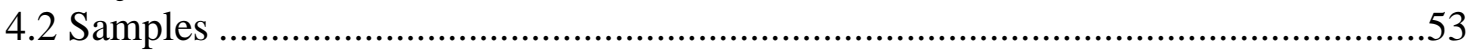

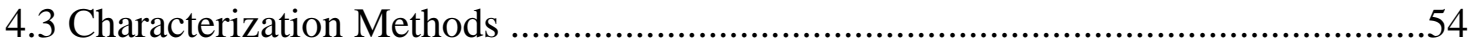

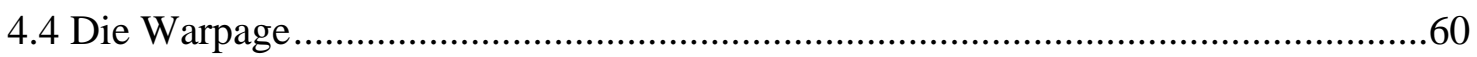

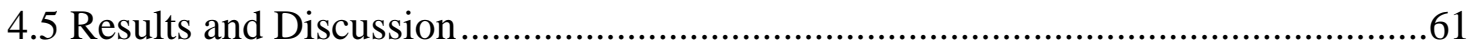

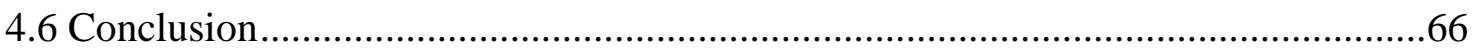

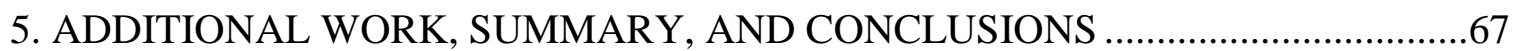

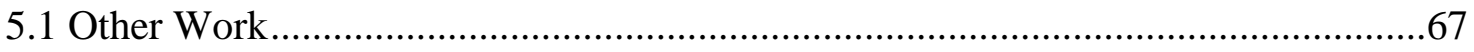

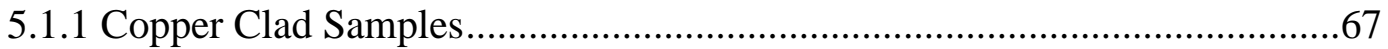

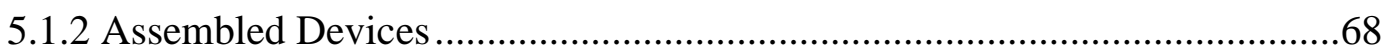

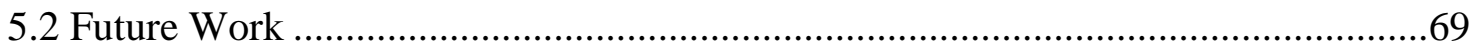

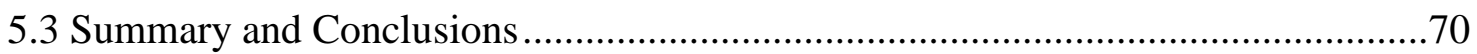

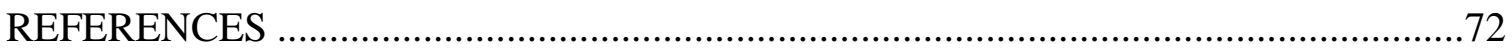

APPENDICES

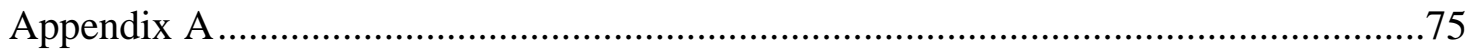

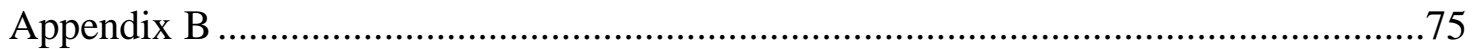




\section{LIST OF TABLES}

Page

Table 1. Summary of Print Variables .........................................................................26

Table 2. Differences in resistance values after Photonic Curing .......................................37

Table 3. Phase 2 Crease Test Resistance Results …………………………...................45

Table 4. Wash Test Resistance Increase Results ............................................................47

Table 5. Resistance Improvements for Convection Oven Tuning ......................................49

Table 6. Matrix of Multiple Convection Oven Run Results...............................................49

Table 7. Final Curing Test Resistance Results ……………........................................50

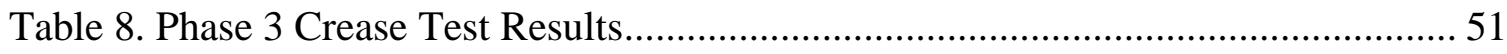




\section{LIST OF FIGURES}

Page

Figure 1. Side view of screen printing process. The various parameters determine

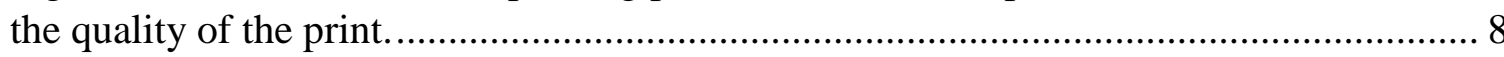

Figure 2. Summary of printing technologies used in printed electronics. .......................... 8

Figure 3. Pattern and Encapsulation method for fabricating flexible substrates on

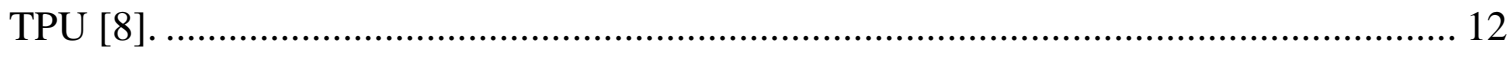

Figure 4.The shape of metallic stretchable interconnections can greatly affect the feasibility of its performance during stretching. The horseshoe shape has long stood as the optimal design for reliable, flexible connections [9] . .......................................... 12

Figure 5. Schematic of dicing before grinding method and its process flow. The final etch step serves to remove dicing damage [12] .................................................... 15

Figure 6. Schematic of characterization methods for determining die strength:

(a) 3 pt. bend test (b) 4 pt. bed test (c) ball-on-ring test (d) ball-on-pad test [12].

Figure 7. SEM image of cross-section of isotropically conductive adhesive on polyimide. The ink has been inkjet printed as well [21].

Figure 8. A 12"x12" vacuum plate was manufactured for holding the samples flat during printing. The plate was taped off to the exact substrate size for better vacuum performance.

Figure 9. Screens provided by Sefar utilized a trampoline mesh along with an emulsion for printing. The 29" frames were provided by Cal Poly GRC department..... 24

Figure 10. Screen pattern showing the long "ladder lines" and the features used for profilometry measurement and analysis purposes.

Figure 11. Four-point sheet resistance measurement probe used to measure sheet resistance of thin films. The two outer probes have a current run through them and the results voltage is measured between the inner two probes.

Figure 12. Close up of profilometer cantilever probe used for measuring print height. The resolution of the Ambios profilometer is in the 0-1 micron range.

Figure 13. Microscope image of spacing of printed footprint traces for PacTech 2.3

die. The spacing of the traces is critical to daisy chain assembly performance. 31 
Figure 14. Pareto chart of effects of printing parameters on final resistance values. Print ink, squeegee pressure, and the interaction between ink, print speed, and print pressure were all significant. 32

Figure 15. Main effects plot for the exterior pattern $2 \mathrm{~mm}$ trace. Based on the data the squeegee pressure is the primary parameter aside from the ink choice.

Figure 16. Pareto chart of significance levels of longest trace. 15b. Main effects plot of emulsion

Figure 17. Profile of square pad showing scooping outline. Based on the un-uniformity it is unlikely that sheet resistance using the 4-point probe is unviable. .... 34

Figure 18. Profile of inner traces of PacTech die footprint. The average height of the traces was estimated and used for analysis.

Figure 19. Side profile of PE873 ink printed on TPU. The large surface roughness of the TPU made it challenging for verifying print height with certain parameters leading to a print roughly $8 \mu \mathrm{m}$ tall and $0.35 \mathrm{~mm}$ wide.

Figure 20. Pareto chart results for significance of PE873 printed traces in relation to overall print height.

Figure 21. Sample of over cured print using photonic curing. Lift off and separation are common failure methods.

Figure 22a. Results from after 10 cycles of crease testing. 22b. Pattern sample showing location of crease on print.

Figure 23. Flowchart of curing test for Phase 2 print experiment. Based on the initial resistance measurements and the reliability test results, one curing methods was selected for moving forward with the project.

Figure 24. Samples were heat pressed onto fabric and creased 10 times along the center of the ladder lines. Samples were creased using a Delrin carpet roller to ensure even pressure throughout the crease.

Figure 25. Samples were washed in detergent for 30 seconds then air dried. A whisk was used to simulate the spinning of a normal washing machine.

Figure 26. Boxplot of resistance results $(\Omega)$ for each curing method used.

Figure 27. Chart comparing resistance results based on print orientation and curing method 
Figure 28. Tape test results showing no peeling of ink. Lines shown are material removed due to scoring with a razor blade. 46

Figure 29. Images of the printed inks 5025 (left) and PE873 (right) show particle sizes ranging from 1-10 $\mu \mathrm{m}$ in size. The images are consistent with the information that the PE873 ink would have larger flake-like particles to allow stretch ability.

Figure 30. Interface of conductive ink and TPU substrate showing the large surface roughness of the substrate. Overall the ink appears to show little spreading after printing.

Figure 31. Top view of PacTech 2.3 die with $10 \mathrm{~mm}$ side length (left). SEM image of flat NiAu bump on PacTech (right).

Figure 32. Profilometry performed on the thinned die revealed a die thickness of roughly $80 \mu \mathrm{m}$. The bump shown is one of the edge bumps on the die.

Figure 33. Cross section of thinned die in SEM with thickness measurement for reference.

Figure 34. Camera image of stylus profilometer measuring the corner of the die which is assumed to have the least amount of warpage across the surface. Prior to beginning the scan off the die the stylus is lowered until contact is made with the die. Occasionally during this lowering the die corner was observed to be pressed down until contact with the stage was made.

Figure 35. Profilometer map depicting the 3 measurement paths taken by the stylus to measure die thickness and overall warpage. The challenge for this measurement is avoiding tracing over any bumps which might alter the thickness data.

Figure 36. Die tapped against the edges of double sided tape allow for ease of measuring without affecting the internal stresses of the die.

Figure 37. Profilometry profile of entire die showing amount of warpage in one linear direction.

Figure 38. Due to the internal stress in the silicon die, warpage can be viewed by visual inspection alone.

Figure 39. Die thickness side profile showing sloping of die warpage and edge thickness of die. 
Figure 40. Descriptive statistics of die thickness. Given a target thickness of $50 \mu \mathrm{m}$ the process does not show consistency in uniformity or mean..................................... 62

Figure 41. SEM image of a thinned silicon die showing the side thickness and its variation across the $10 \mathrm{~mm}$ side of roughly $10 \%$.

Figure 42. Original profilometry profile of entire die showing amount of warpage in one direction. $\mathrm{Z}$ axis results include both warpage and die thickness making it difficult to determine the exact values for each.

Figure 43. By leveling the edges of the die measurements at zero the overall warpage can be characterized as the height above the $\mathrm{x}$ axis.

Figure 44 . Trend showing die thickness vs. die warpage. Roughly $20 \%$ of the die received contained significant amounts of warpage with thinner than expected thicknesses.

Figure 45. Copper clad samples on TPU as prepared by supplier. The copper shows noticeable restrictions on the substrate when small forces are applied.

Figure 46. SEM images of $\mathrm{Cu}$ clad samples on TPU. Copper thickness and TPU thickness measured support expected thicknesses (left). The rough interface and porous TPU surface may pose a challenge for future assembly tests (right). 


\section{CHAPTER 1. INTRODUCTION}

As the world of electronic devices continues to develop and the bounds of current manufacturing methods begin to emerge, new and exciting technologies have surfaced on the horizon. One of these potential new technologies is printed electronics. Imagine having a jacket that has integrated sensors and devices that can monitor heart rate, steps, environmental conditions, etc., all printed directly onto the fibers that make up the article of clothing. While much development has taken place in the area of printed electronics, the field as a whole is still far behind in terms of typical capabilities expected from a CMOS based electronic device. It is for this reason that a new field of electronics has begun to emerge: Flexible Hybrid Electronics (FHEs). These FHEs plainly put combine a traditionally fabricated CMOS based device with a flexible circuitry substrate. By thinning the silicon die below thicknesses of 75um, the silicon becomes flexible to an extent and is able to be attached to a flexible substrate. Likewise, through the use of printed, conductive inks, one can avoid the rigidity that comes with using traditional copper traces. Although the extent of malleability of fully printed devices may still not be possible with FHEs, many of the sought-after device applications can be achieved by removing the aforementioned rigid boundaries and allowing for functional and flexible product applications.

\subsection{Statement of Research Question}

The primary goal for this research will be to develop and test methods for characterizing both printed samples of conductive inks and thinned samples of silicon device die. Likewise, the research will investigate reliability tests for assembled devices and develop 
and optimize methods for scalable manufacturing practices with FHEs. A list of subquestions on the main research question follows:

1. What are the optimal parameters for measuring the quality of a conductive ink print?

2. At what frequency and depth must production print samples be tested for producing a reliable manufacturing chain?

3. What are outside parameters not directly related to printing that can affect conductivity performance (i.e. environmental conditions, age of product, cyclical use, etc.)?

4. What are the optimal methods for the thinning and dicing of silicon ICs and what metrology must be taken on the assembler's part to ensure quality deliver from suppliers?

5. What are the optimal characterization methods for determining sufficient die surface roughness and how can those methods be implemented into a manufacturing system?

6. What is a primary testing method for verifying assembled device function that is both economical and practical in a manufacturing line?

7. What are the most common sources of failure in print quality, die preparation, and device assembly and what metrology is necessary to reduce and avoid producing such defects?

The research on these questions will seek to provide industry with the knowledge necessary to begin pre-production planning on commercially available products with FHE technology. Furthermore, this characterization research will further expose 
individuals to the potential of FHE devices and the ability to use currently in place technologies for manufacturing and testing. The importance of this research is such that commercially viable FHE devices cannot exist if product yield is insufficient for profitability. By expounding on characterization techniques and developing best known methods for assembly testing, the overall yield experienced by a manufacturer will increase, thus leading to a sooner time to market for many applications.

\subsection{Issues and Assumptions}

The most prevalent issues associated with this product are the quality of the prints and die produced from manufacturing. These quality issues can be either inherent to the project assembly processes in house, or they can be derivative of a supplier's quality issues. As for the printing process the issues being researched include but are not limited to, sufficient presence of conductive particles/platelets in the cured ink, sufficient conductivity for device performance, optimal height of deposited ink on the surface to provide functionality but not inhibit flexibility, and sufficient adherence of the ink to the substrate itself. Assumptions include the ink supplied is the best possible ink for the application and that there are no issues from the supplier (no characterization on supplied ink as is will occur); the best print will be the one with the lowest resistivity while maintaining stretch ability; no additional print layers (carbon underlay, encapsulation, etc.) will be used to aid the performance of the print. These assumptions are justified in that electronic device performance is inherently driven by low resistivity, the ink suppliers already have extensive product testing prior to customer delivery, and that the addition of subsequent print layers will only assist in the performance of the print and 
therefore are outside the scope of the project. Issues for consideration regarding the thinned silicon die include but are not limited to: optimizing surface roughness to ensure no premature crack propagation, uniform thinning of the die to avoid warpage or variable mechanical properties, controlling the thickness of the final die over the entirety of the wafer to ensure enough tolerance for final device performance, and finally verifying the die are indeed thin enough to provide the required flexibility for device performance. Assumptions include: the performance of the supplied daisy chain ICs is equivalent to that of a fully functioning CMOS device in regards to degraded performance related to thinning; $75 \mu \mathrm{m}$ is the optimal target for thinning in regard to the desired flexibility and the bump/circuit design does not affect this target; the die as supplied by supplier are ideal for testing and the thinning process from the supplier is the best available option for the scope of this project. These assumptions are verified in that each of them is either directed by previous research or lies outside of the scope of this project. Furthermore the overall assumption is made that the equipment available at Cal Poly is of high enough quality and variability that a manufacturing partner could easily replicate similar testing processes.

\subsection{General Approach}

The approach of this project will seek to be as quantitative as possible. This will prove to be difficult in the area of print characterization as traditional print quality is inherently qualitative. Therefore, a mixed methodology will be utilized, being sure to provide as much quantitative data as possible, yet supporting with qualitative analysis when necessary. Experimental processes will be optimized and repeated in order to provide 
enough samples to develop a reliability and testing model that provides support to the conclusions of the research. The project will start by first optimizing the print conditions based on characterization research. Alongside this the research will analyze the thinned die provided by the supplier to ensure quality product to continue towards assembly. Lastly the majority of experiments will focus on characterizing design performance post assembly and researching with industry partners to implement a testing plan that is both economical and feasible for a scalable manufacturing process.

This approach fits in with previous research in that it will supplement the methods already described in academia for characterizing thinned silicon ICs. This approach will however extend the state of said research as it seeks to bridge the gap to an industry partner looking to begin commercial manufacturing of such devices.

An outline of the chapters is as follows:

- Chapter 1 - Introduction

- Chapter 2 - Background and Literature Review

- Chapter 3-Characterization of Printed Circuits on TPU Substrate

- Chapter 4 - Characterization of Thinned Silicon IC Die

- Chapter 5 - Additional Work, Summary, and Conclusions

- References

- Appendices 


\section{CHAPTER 2. BACKGROUND AND LITERATURE REVIEW}

\subsection{Background}

The general topic of this thesis is regarding the area of flexible hybrid electronics. Prior to understanding the details of this research, it is important to understand the history of flexible hybrid electronics (FHEs) and the terminology associated with their use. The following section details the specifics regarding FHEs, traditional printing terminology, and traditional electronic terminology.

\subsubsection{FHEs}

The term flexible hybrid electronics was developed to carve a distinct niche for devices being researched. The term flexible, though vague in magnitude, is generally regarded as something that can bend, stretch, and flex within reason and still maintain functionality. Typically bending is measured by a minimum bending radius as defined in ASTM standard E290, with a range of less than a micron to upwards of several centimeters. The term hybrid is explanatory of the two currently established technologies being brought together for this new area of technology. The two technologies involved are printed electronics, and traditional silicon based electronics. Each technology has properties of value, that when combined, open new opportunities for product development in the wearable electronics marketplace [1]. The basis for producing FHEs is to harness the flexibility of the polymer substrates and conductive inks of printed electronics, and combine them with the functionality of advanced CMOS based sensors and processors. It is therefore fundamental to understand the background of both printed electronics and traditional electronics when analyzing this research. 


\subsubsection{Printed Electronics}

The printed electronics industry maintains over a decade of established presence as an industry and continues to advance with new research into higher conductivity and greater flexibility with its inks. In terms of printing with conductive inks there are several methods which have been proven useful. The first and most established is inkjet printing [2]. Inkjet printing provides the benefit of low production cost, along with high resolution for printing fine features. One challenge though is maintaining consistency of the ink, as it is common for the conductive particles to clog print heads, thus diminishing the benefits. The second, most potentially beneficial process, is using flexography or flexographic printing. Flexo, as it is commonly referred to, utilizes a continuous, roll-toroll process that involves cylindrical rollers which deposit ink onto flexible polymer sheets [3]. The costs savings is exponential as circuits can be printed rapidly and continuously. The last method of printing and the one being used in this project is screen printing. Screen printing uses a stencil like process to transfer a pattern through a mesh outline onto a single sheet. Ink is deposited on the edge of the desired pattern and a squeegee is dragged along the surface to push the ink through the pattern. Screen printing is popular among discrete printing operations, specifically relating to textiles and clothing. Important parameters include the mesh material, mesh opening size, emulsion thickness, emulsion hardness, print gap, print speed, print pressure, along with many additional factors (Figure 1). When comparing the various printing methods together, screen printing excels in the area of print height, as it can apply large thicknesses of ink in a single pass (Figure 2) [4]. Part of this project will seek to optimize several of these parameters for the specific ink and substrate being investigated. 
continues to grow as an industry these rigid silicon chips have become a challenge and hassle to new devices. Likewise, the current manufacturing process for silicon fabrication (photolithography, deposition, etching, implantation, etc.) requires large capital investments and inhibits device manufacturers from producing small batch quantities in a cheap and timely manner. Furthermore, the use of printed circuit boards (PCBs) as the physical attachment method for electrical interconnection most commonly uses rigid composite based boards with little flexibility. PCB suppliers have developed partially

flexible based boards using polyimide as a substrate, however the restriction of the solid copper traces does not allow for large strain values or functionality under complex loading scenarios.

\subsubsection{Project Goals Overview}

Plainly put, the objective of this project is to develop assembly processes for ultra-thin silicon integrated circuits and sensors for textiles and to evaluate the reliability of those processes for scaled manufacturing. Specifically, the project will focus on a manufacturing strategy with regards to device integration and packaging, followed by reliability testing. The current Manufacturing Readiness Level (MRL) for FHEs is estimated at MRL 4, with the end goal of this project to bring it to an MRL 6/7 level (Appendix A). 


\subsection{Literature Review}

\subsubsection{Previous Work Done}

Prior to this study, several application based experiments have set forth to utilize FHEs to build devices that can be incorporated into human monitoring systems. Such devices make use of the benefits of flexibility and complexity in the thinned ICs in order to provide easily accessible data about various functions. One example of this is an athletic shirt designed to incorporate a 12 lead ECG and transmit the data back to the user's smartphone via Bluetooth [5]. By using a chip, antenna, and transmitter of less than 2.3 $\mathrm{mm}$, the shirt can be worn without discomfort. It is anticipated that through further FHE development, significant improvements in size and functionality are possible.

Along with flexibility, another feature of these printed electronics is optical transparency. By combining transparency with flexibility researchers are able to develop new medical devices for monitoring human systems directly [6]. In one particular study researchers used a $1 \mu \mathrm{m}$ thick parylene sheet substrate with thin film transistors (TFTs) to fabricate a contact lens with embedded processing capabilities. The end goal is to use embedded strain gauges to detect and diagnose glaucoma disease.

\subsubsection{Printed Electronics Work}

The primary driver of printed electronics is the conductive ink used. Extensive research as gone into developing these conductive inks using a silver based ink that has been inkjet or screen printed [2]. The development of nanosilver ink further enhanced the conductivity of such devices, due to the decreased melting point of silver nanoparticles and the ability to manufacture inks with higher weight percentages of silver. Furthermore, 
by using silver nanoparticles, smaller features are enabled ranging down to the 10s of microns [7].

In terms of polymer substrate, a Thermoplastic Polyurethane (TPU) film is being investigated as an ideal solution for human monitoring systems. TPU has previously been evaluated in lab environments as a viable solution for using silver in stretchable electronics applications [8]. TPU maintains advantages over common polyester (PET) in its stretchable and breathable properties. These benefits ensure comfortability when wearing a garment integrated with printed electronic circuits. Furthermore, it has recently been investigated of using TPU as a substrate for direct screen printing of silver conducting inks. The low surface roughness of TPU provides reasonable printing surface and enables significantly higher failure strain values compared to printing on fabric directly [8]. The tests also reveal the importance of encapsulating the printed ink with a stretchable dielectric material. This encapsulate becomes even more necessary when performing wash cycle tests, those of which yielded an endurance of 100 wash cycles [8]. Geometry of the traces also was tested and provided increased performance based on a sinusoidal line shape (Figure 3). It's important to note that the traces in this sample had a width of $1 \mathrm{~mm}$, whereas the traces in this study will be as thin as $150 \mu \mathrm{m}$. 


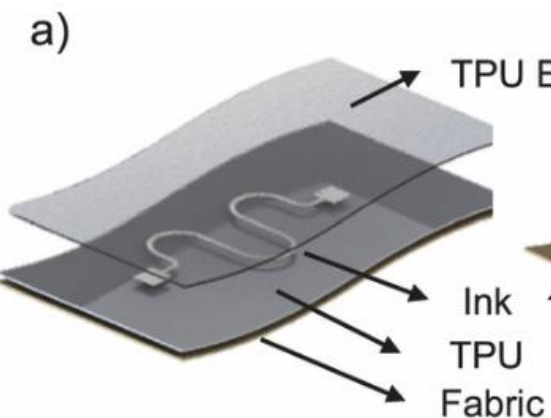

b)

c)

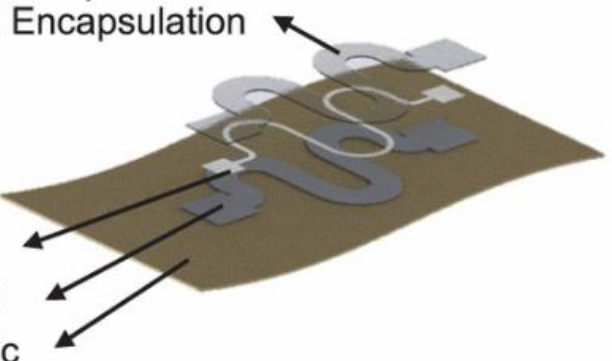

d)

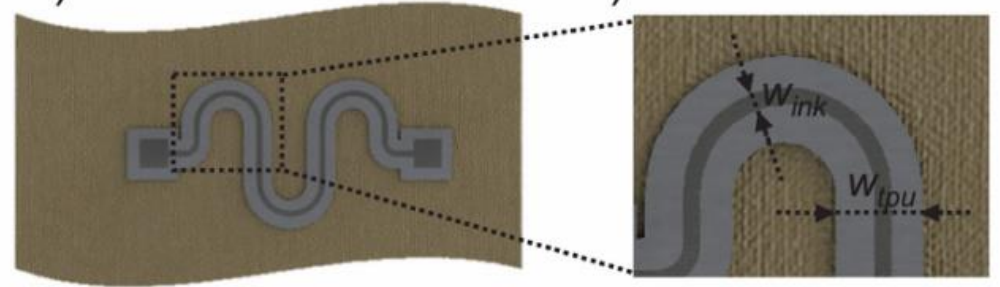

Figure 3. Pattern and Encapsulation method for fabricating flexible substrates on TPU [8].

When considering the geometry of the flexible traces, it is key to note that original geometric considerations where developed using copper plate on polymer substrates.

These developments led to the accepted geometric shape of the horseshoe design (Figure 4). For copper, suggestions have been made to break the flexible traces into a multi-track horseshoe design, which in turn reduces the large strains experienced by a wider trace [9]. Currently there is no production of using copper with stretch ability up to $100 \%$.

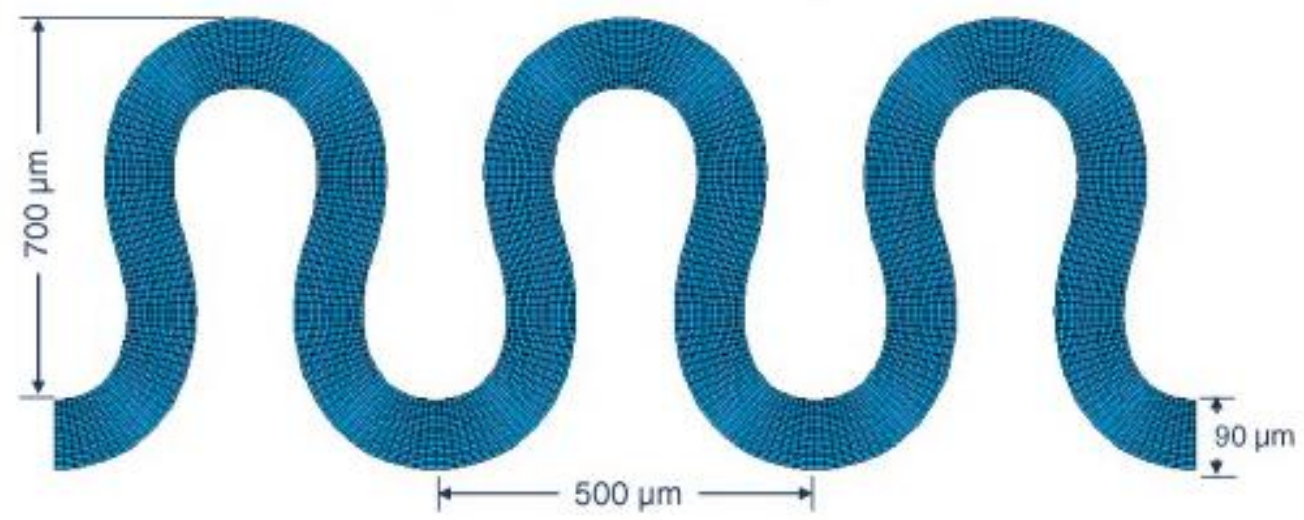

Figure 4.The shape of metallic stretchable interconnections can greatly affect the feasibility of its performance during stretching. The horseshoe shape has long stood as the optimal design for reliable, flexible connections [9]. 
It is important to note that flexible copper based substrates have been around for quite a while. Using a polyimide based substrate, copper is able to be etched and patterned similar to traditional PCB manufacturing systems. Polyimide provides a good choice as it can withstand the higher temperatures used for soldering, as well as maintaining a coefficient of thermal expansion close to that of copper [10]. Much development has gone into these applications, with the result being a mildly flexible, well proven application for electronics use. Currently the limitations of such technology lie in that the copper itself can only be mechanically deformed to a certain degree. Furthermore, there are well documented issues relating to adhesion between the copper and polyimide layers [10]. It is worth noting that any potential change in polymer substrate would likely have these issues as well.

\subsubsection{Thinned Silicon Research}

The second primary area of research is into how to best thin down silicon wafers to produce die that are complex in function, but still flexible enough to support their wearable applications. It has been shown in lab environments that it is possible to thin silicon down to as low as $6 \mathrm{~nm}$ in thickness [11]. While this may not be the thickness required for this application of study, it is cited merely as a reference for the possibilities of silicon processing. Traditionally silicon has been thinned down to $100 \mu \mathrm{m}$ range for use in stacked chip assembly process and 3D through silicon via architecture [12]. The proposed die thickness for the project is between $30-75 \mu \mathrm{m}$. These are the main boundaries of the ultra-thin silicon die category, and provide the target from where to start from. Similar devices have been fabricated using die as thin as $25 \mu \mathrm{m}$, with total 
package thicknesses of sub-100 $\mu \mathrm{m}$ range [13]. These devices rely on sputtered metal layers however, which limit the overall flexibility of the application. A key factor when considering flexibility is how thin the requirements are. This is crucial, as the thinner the die the more susceptible it is to fracture. Likewise, any encapsulation and attachment methods are likely to increase the total dimension of the package and decrease flexibility. A key factor when considering these die is the method of handling. Due to the paper-thin nature of the die, precision handling tools are required to maneuver and bond the die to the substrate. Many options and configurations exist for handling and the exact handling tool depends greatly on the application required [14]. Typically, the die is picked via a vacuum head and carried within the machine. This vacuum seal relies heavily on the flatness of the die and any warpage has the potential to cause issues during manufacturing [15]. Along with handling is the issue of how to effectively dice the wafers into the respective die. Challenges include the fragility of the die due to thickness, and the requirement that the wafer be completely flat and oriented. Typically, wafers are diced using a saw blade with diamond edges. Other methods include using a two-blade system, which uses a large blade to make an initial shallow trench, followed by a thin blade to cut through just the silicon. This method significantly reduces the propagation of surface cracks. One development that currently leads the industry in terms of dicing technique is the dice before grinding method [12]. This method uses a diamond saw to cut trenches in between the silicon die, deeper than the end goal thickness, but not all the way through. The wafer is then bonded to a wax carrier and flipped over for grinding purposes. As the wafer is ground down the trenches are further exposed and the die are now free floating (Figure 5). Further research has shown that using a plasma laser to etch the trenches, as 
opposed to diamond saw, leaves significantly less surface cracks and thus less chance of crack propagation during [12].
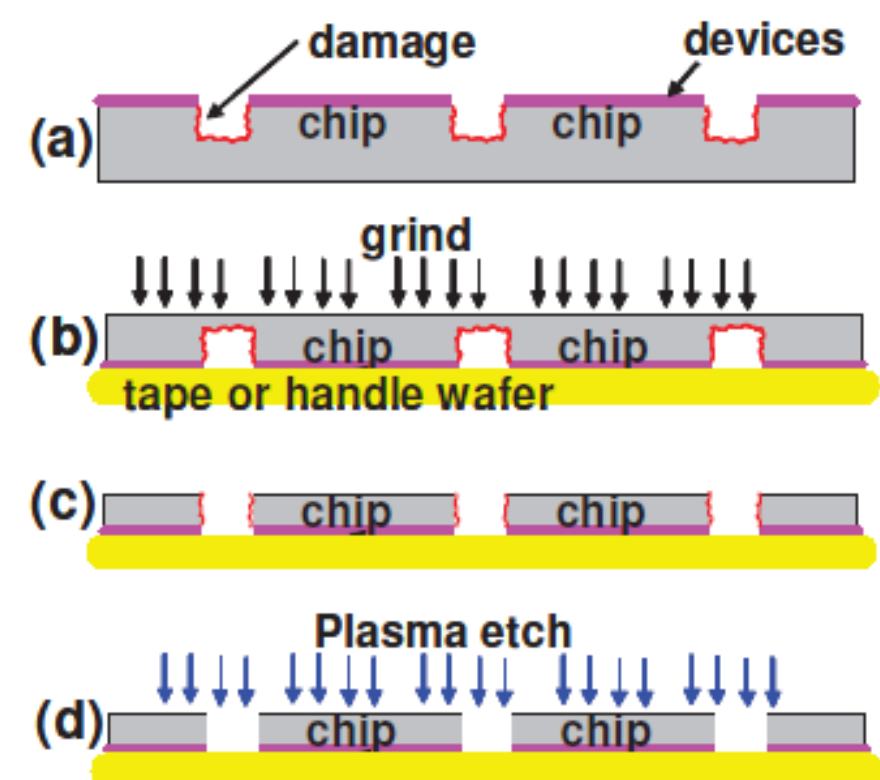

Figure 5. Schematic of dicing before grinding method and its process flow. The final etch step serves to remove dicing damage [12].

Aside from the dicing by grinding method, additional research has gone into using high powered lasers to cut through already thinned wafers [16]. By thinning the wafer down without any induced mechanical deformation there is theoretically a more uniform thickness across the wafer. By having the silicon extremely thin at this point it is then possible to cut through the silicon using the laser energy without damaging the internal microstructure. Results have shown increased strength in these die, however the process must be precisely controlled and is significantly more expensive [16].

In terms of characterizing the thinned die, four main methods exist: particle defects, total thickness variation (TTV), wafer bow, and fracture strength. Particle defects, TTV, and wafer bow are typically characterized on the wafer level, however the TTV can be characterized by means of the variation in thicknesses of die received from the dicing and thinning contractors. Typically wafer bow is measured via optical or laser based methods 
[17]. It utilizes a refence plane and then records the difference in height between the plane and rest of the wafer. It is also possible to map the bow on a single die using optical tracing and plotting of the displacements from the reference plane to measure die warpage. Additional methods for measuring die thickness and warpage include stylus profilometry and optical ellipsometry.

For measuring fracture strength there are four primary methods of analysis: 3 point bend test, 4 point bend test, ball on ring, and ball on pad (Figure 6) [12] [18].
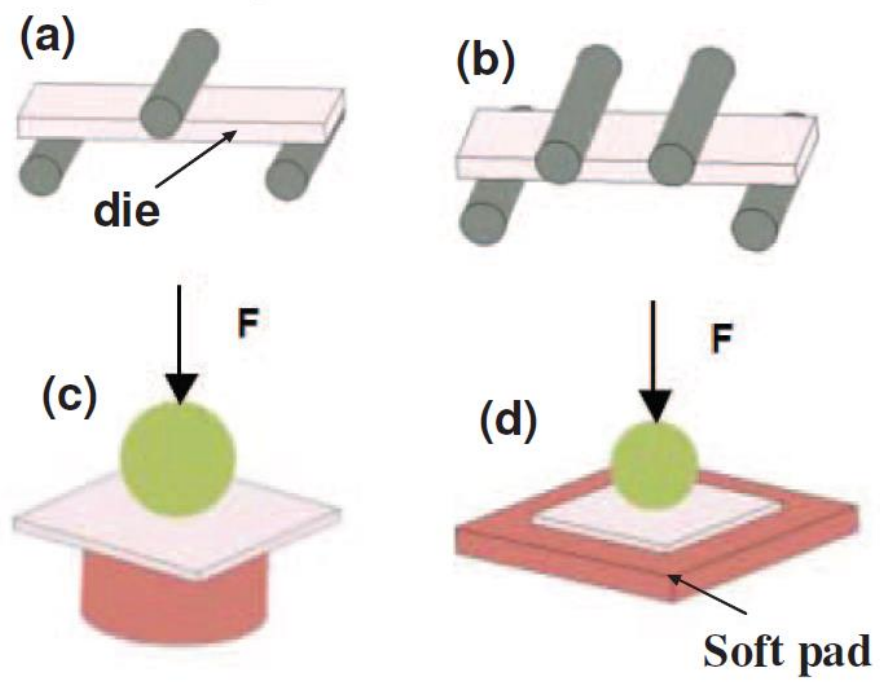

Figure 6. Schematic of characterization methods for determining die strength: (a) 3 pt. bend test (b) 4 pt. bed test (c) ball-on-ring test (d) ball-on-pad test [12].

These are all destructive testing methods and therefore large sample sizes are seldom used for fracture testing. Weibull statistics can be used to estimate overall yield from these tests [12]. Both the bending tests will fracture due to edge and surface damage; however, the ball tests are likely to only fracture due to surface defects.

The main source of fracture is microcracking left behind in the thinned die. Depending on the quality and fineness of the grinding pad, micro-cracking can occur on the surface of the die. This cracking occurs within $1 \mathrm{~nm}$ to $1 \mu \mathrm{m}$ deep from the surface. It is necessary 
to remove such defects in order to reduce the chances of fracture. Several methods used for this include a dry polish, a CMP process, a plasma etch, or a thermal anneal.

\subsubsection{Attachment Methods}

A key part of the research conducted will be on the methods by which the ultra-thin die is attached to the polymer substrate. Previous research has shown several methods to be viable for attaching the die while still maintaining flexibility. The goal is to determine which method offers the best options for a scalable assembled device based on reliability and manufacturability. The three options being considered are isotropic conductive adhesive (ICA), anisotropic conductive adhesive/film (ACA/ACF), and photonic sintering.

Isotropic conductive adhesives are a category of somewhat established, conductive adhesives that utilize conductive particles in a polymer matrix, usually epoxy. Being isotropic they conduct in all directions and are commonly used in applications of chip contacting and bonding electrically conducting SMDs [19]. The primary issue that arises when considering ICA for use in attaching SMDs is the material selection [20] [21]. The substrate and precise ink parameters are key to determining which ICA will perform best in any application. Viscosity, print speed, and stencil opening are all key parameters that affect electrical performance as well as manufacturability and placement precision [21]. By using ICA along with nanoparticle silver ink and a polyimide substrate it is possible to achieve performance comparable to traditional copper based PCBs, however reliability is a large concern. Unlike solder, most failure in conductive adhesives is due to delamination between adhesive and the component terminal (Figure 7). Temperature 
cycling, most commonly used in industry, is used to test thermomechanical reliability and simulates the long-term reliability due to CTE differences [21]. ICA reliability has tested with both chip resistors and BGA components with cracks occurring largely due to the CTE differences, most commonly at the component interface. It is well-known that mechanical shock performance of ICA is far worse than solder [22].

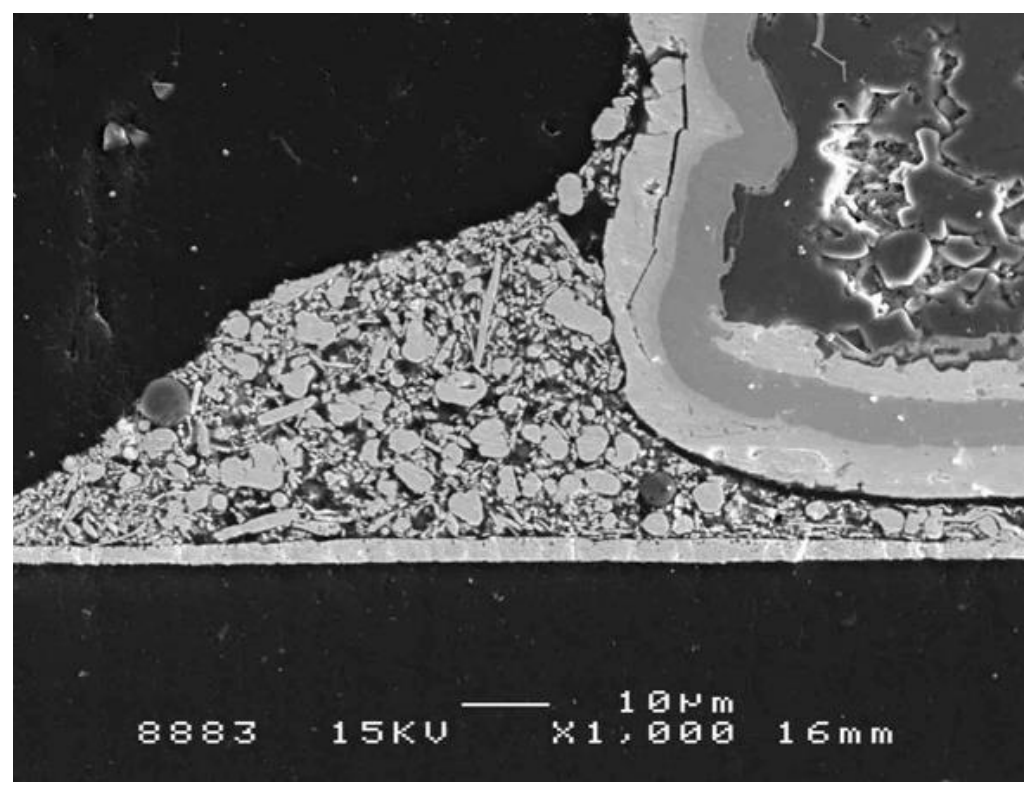

Figure 7. SEM image of cross-section of isotropically conductive adhesive on polyimide. The ink has been inkjet printed as well [21].

ACA, on the other hand, has been used for many years in electronics assembly. It can be categorized in either a paste or film form. ACA utilizes conductive particles on the micron scale that, upon pressurization, conduct in the direction of the applied force. This allows for fine pitch assembly without shorting [23]. ACA has been developed for use with traditional PCBs, however there is little cost benefit for not utilizing more developed lead free solders [24]. Resistivity of ACA has been estimated (Equation 1) where $\rho_{B}$ is the resistivity of the sphere material, $n$ is the number of contacts within the contact area $A$, $\kappa$ is the shear yield stress on a single contact sphere of radius $R_{B}$, and $\sigma$ is the pressure applied to the joint [25]. Key factors that influence the bonding quality include curing 
temperature and time, ramp rate, alignment, bump height, and substrate uniformity [23].

Both oxidation and moisture absorption are known causes of joint failure in ACA.

Overall there remains many areas where ACA development and understanding its lifetime can be investigated.

$$
\rho=\frac{A_{\rho_{B}} \sqrt{\frac{6 \pi n \kappa}{\sigma A}}-\frac{1}{R_{B}}}{4 \pi n R_{B}}
$$

Similar to ACA, ACF relies on mechanical normal force to enable conduction. The benefit of the film is simplicity of application and ability to cover large areas uniformly. Tests for judging the reliability of ACF joints include temperature and humidity cycling, similar to that of other adhesive attachments [26]. The key factor with ACF is the contact resistance introduced. The applied pressure must be sufficient to allow particles to interact with the conductive surfaces, despite the presence of the film material. Flexibility tests have shown that bending can both increase and decrease the resistance of the joint, likely due to the surface area in contact with the particles [26].

The third and final attachment method that holds potential for use in FHE assembly is the photonic soldering method. This method uses traditional solders, however relies on an innovative process flow to allow compatibility with flexible substrates. The problem with traditional solder reflow for use in FHEs is that, depending on the alloying elements, some solders must be maintained at temperatures above $200^{\circ} \mathrm{C}$ to allow the liquidous transformation to occur. This temperature however is above the maximum service temperature of many of the substrates being used for FHE development of $120-150^{\circ} \mathrm{C}$ [27]. Photonic soldering solves this problem by using a high-energy xenon light which pulses to provide energy to the assembly. By pulsing the light, it is possible to avoid 
energy absorption in the substrate, while still elevating the solder temperature to the necessary requirement [27]. Photonic soldering has also been shown to be useful in curing the conductive inks, by providing a quick and reliable curing method that is compatible with roll to roll systems for manufacturing. It is important to note that certain substrates will absorb more energy than others, therefore extensive testing of the materials is required prior to validation.

\subsubsection{Research into scalability of FHEs}

The key to any new technology development is how likely it is to break through into the commercial market. It was estimated that prior to the beginning of this experiment FHEs possessed an MRL 4 rating, with the goal to bring them to MRL 6/7. In order to determine what the best method for expanding this technology it is worthwhile to compare other technologies that have previously scaled and established themselves in the marketplace. By looking at these precedents it is possible to better formulate a future development plan and determine methods for gauging further success.

As mentioned the current scale of the FHE market can be defined as being established in the lab setting. Multiple papers have been published regarding the viability of FHE devices and large companies are beginning to take interest in the market as a means for decreased size and weight for future devices [28]. However there still exists many notable deficiencies for bringing this technology to full scale. Most prominent is the challenge to reliably attach SMDs onto flexible circuits [29]. This is because there is a lack of fundamental understanding of the behavior of response of each component under thermal, mechanical, and humidity loadings. Furthermore, some modeling work on conventional 
flexible CMOS devices has been performed, however, no previous models exist for printed flexible substrates with SMDs. The printed flexible substrates pose new challenges for modeling, such as adhesion at the interface, integrity of printed traces, and hydroscopic behavior under humidity. The residual stress in printed substrates will also have an impact on interconnection reliability of the SMDs [30]. There is a benefit though for FHEs in that most the technology applications they will be used for has already been developed and therefore the only new cost associated with the Si chips is to thin down previously established designs [28].

A key component when discussing scalability is the adaptability of the manufacturing system and its components [31]. Almost all established manufacturing systems have survived the ebb and flow of the market by remaining flexible to dynamic customer needs. The benefit here for FHEs, especially those in a roll-to-roll environment is that the print industry consistently changes the product being produced, often multiple times a day. Therefore, adapting to new designs falls more so on simply configuring material properties and enabling those to work under given manufacturing parameters. Four key steps to establishing scalability include solving the problem in less time, solving larger problems in the same time, obtaining sufficient memory to solve the given problem, and continually seeking to solve even larger problems regardless of time [31]. If the FHE market is to establish itself it must not get solely focused on the short term of simply making the previously existing devices flex, but rather seek out chances to develop comply new devices that could further revolutionize electronics into the future [32]. 


\section{CHAPTER 3. PRINT DESIGN AND DEVELOPMENT}

\subsection{Print Phase 1}

\subsubsection{Objective}

The objective of the first phase print development experiment was to analyze the factors involved in the screen printing process and to characterize the deposition of conductive silver inks onto flexible substrates. For the experiment a $2^{4}$ factorial design investigated the effect of four key factors (emulsion, silver ink, squeegee pressure, and print speed). Response variables included resistance readings on straight, dog-bone lines, sheet resistance on square pads, z-axis print height, and inner trace widths.

\subsubsection{Experiment Design}

\subsubsection{Equipment and Materials}

The following equipment and materials were used in this study: the primary piece of equipment used for screen printing was a DEK Horizon 03i printer. One important thing to note is that the printer does not have automatic calibration, therefore calibration must be manually set for each new squeegee installation. A print-flood printing method will be used in conjunction with a DEK squeegee (200 Blue) and a DEK 265 flood bar (200 $\mathrm{mm})$. This squeegee choice was made after failed attempts on the first print phase to optimize a JNJ 12" replaceable squeegee system. Snap-off distance was fixed to a value of 3 mils. The printer was outfitted with a 12" square vacuum plate to maintain suction on the flexible substrate during the printing process (Figure 8). 


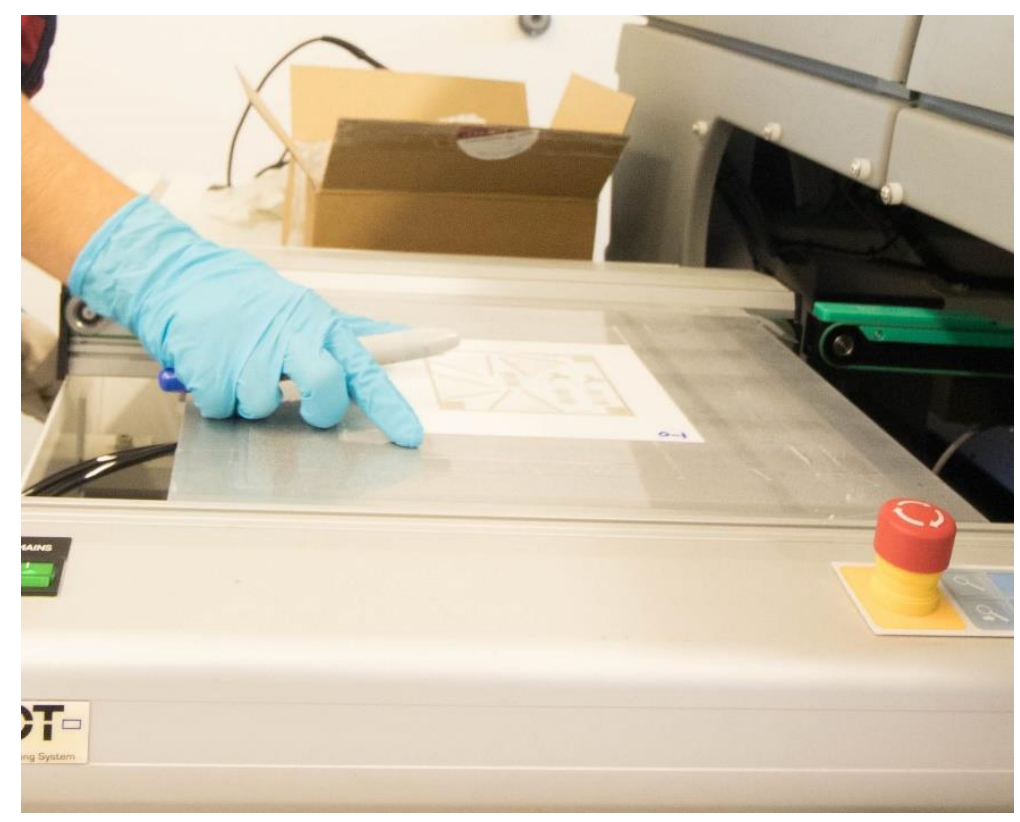

Figure 8. A 12"x12" vacuum plate was manufactured for holding the samples flat during printing. The plate was taped off to the exact substrate size for better vacuum performance.

A humidifier set at $100 \%$ was placed directly next to the DEK machine, providing water vapor into the printing area. This was based on prior knowledge that a more humid environment is beneficial to print success and preventing ink from drying in the fine features of the screen.

The DOE utilized two different screen emulsions, both with the same screen parameters. These screens were 29 in square, metal tape on polyester stretch, with a 400 mesh stainless steel trampoline print area by Sefar (Figure 9). 


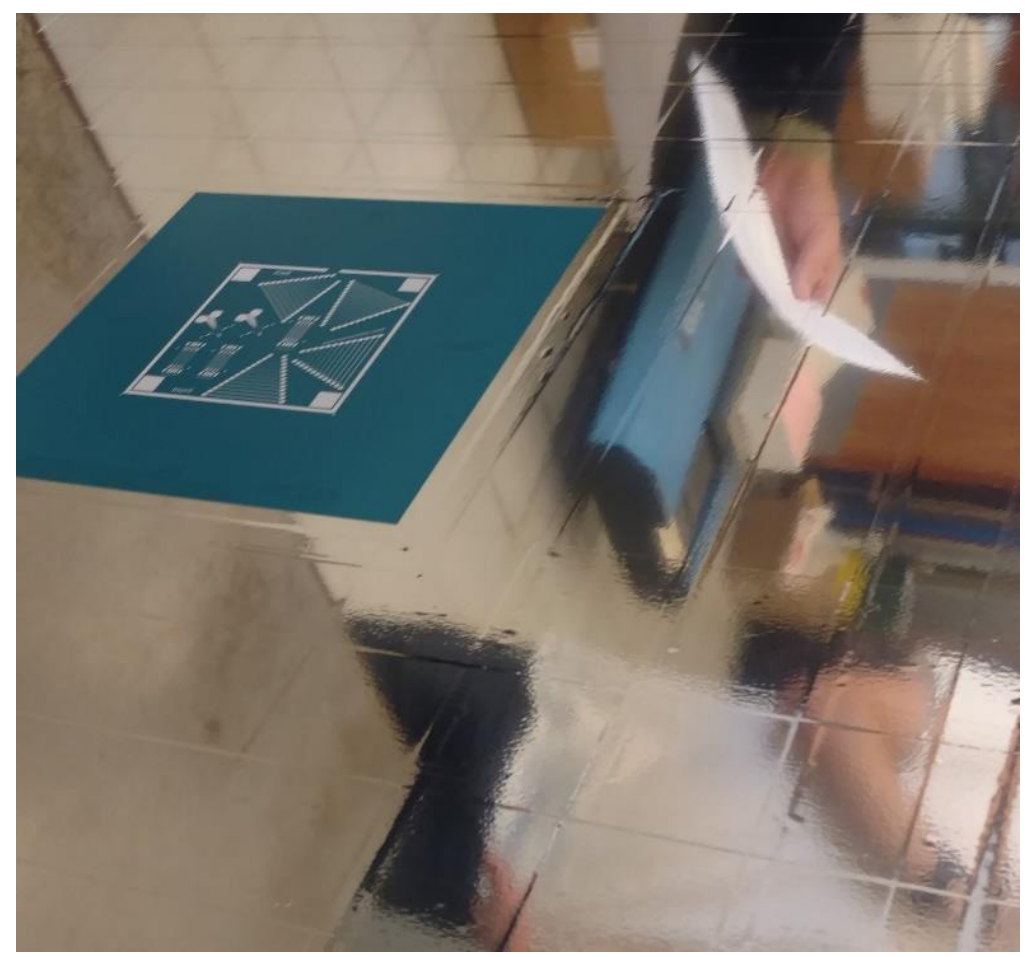

Figure 9. Screens provided by Sefar utilized a trampoline mesh along with an emulsion for printing. The 29" frames were provided by $\mathrm{Cal}$ Poly GRC department.

The substrate used is a Thermoplastic polyurethane (TPU) provided by Bemis corp. The substrate is approximately $75 \mu \mathrm{m}$ thick and is provided with a plastic backing to provide structural support during the printing process. Samples were cut to 6" square size with a reduced printing area of 4" square to increase efficiency. Lastly an Espec box oven was used for curing of the samples after printing. The oven holds six samples at a time located over three different shelves. Samples were first tack cured for 5 minutes at $100^{\circ} \mathrm{C}$, then fully cured later for 30 minutes at $130^{\circ} \mathrm{C}$.

\subsubsection{Design}

The following four parameters were used for the factorial design of this experiment (Table 1).

1. Emulsion. Emulsion can be described as the stencil used for transferring the desired pattern to the substrate. Emulsions start out in either a liquid or sheet form and are 
patterned using UV light to the desired print pattern. The emulsion is then attached to the bottom of the stretched screen, with the intent that it will come into contact with the substrate during printing. For this experiment two different emulsions were chosen: CP and S24.

2. Silver Ink. Although it is already decided that a stretchable ink from DuPont will be used for the final assembly, it was of interest to still print with a highly conductive, non-stretchable ink in order to compare the results and determine the success of the print overall. For this reason, the 5025 silver particle ink from DuPont was chosen, given its past success with printing at Cal Poly. The primary stretchable ink recommended for this study was PE873 silver flake particle ink, also from DuPont. The resistance of the traces is expected to increase with the PE873 ink, however by design it will be able to flex and bend without fracturing on a flexible substrate.

3. Print speed. The third factor under investigation is print speed. By altering the speed at which the squeegee moves across the screen, the print quality is affected due to changing flow dynamics of the ink dependent on the applied force of the print. Based on machine capabilities, previous print tests, and conversations with the DEK technicians, print speeds of $50 \mathrm{~mm} / \mathrm{s}$ and $150 \mathrm{~mm} / \mathrm{s}$ were chosen.

4. Print Pressure. Print pressure is defined as the force applied by the squeegee onto the printed surface when moving across the screen. Many discussions on ideal pressure were conducted, with final considerations given to pressures of 2 and 4 $\mathrm{kg} / \mathrm{cm}^{2}$. One important note is that squeegee pressure can be potentially altered based on the initial gap between the squeegee and surface. Given that our calibration is manual, extra care will need to be had when switching squeegees in 
the future. It is also important to note that as low a squeegee pressure as possible is desired, given that higher pressures will cause squeegees to wear out faster as they a dragged along the mesh surface, even more so with stainless steel meshes. The minimum print pressure of the DEK is $2 \mathrm{~kg} / \mathrm{cm}^{2}$ which dictated the setting limit. Table 1. Summary of Print Variables.

\begin{tabular}{|c|c|c|}
\hline Emulsion Type & S24 & CP \\
\hline Silver Ink & DuPont PE873 & DuPont 5025 \\
\hline Squeegee Pressure & $2 \mathrm{~kg} / \mathrm{cm}^{2}$ & $4 \mathrm{~kg} / \mathrm{cm}^{2}$ \\
\hline Print Speed & $50 \mathrm{~mm} / \mathrm{s}$ & $150 \mathrm{~mm} / \mathrm{s}$ \\
\hline
\end{tabular}

In order to further analyze the factors and account for potential outliers, a replication factor of 3 was chosen, bringing the total number of samples produced to 48 . The run order can be summarized as partially random. First the samples were printed based on ink choice, followed by emulsion type, with the print speed and pressure settings randomized. All 5025 ink samples were printed, first with the S24 then the CP, followed by PE873 using S24 then CP.

The print pattern included various features designed to provide feedback of the print quality and functionality (Figure 10). Squares in each corner were used for sheet resistance measurements and provided feedback on the variability across the entire pattern. Expanding dog-bone "ladder" lines of decreasing length and width allowed for measurement of resistance values at various angles in relation to print direction, likewise an outside trace of $2 \mathrm{~mm}$ thickness provided a summary resistance value for analysis. Lastly footprints of the PacTech 2.3 die were used for trace width and spacing measurements. These footprints had varying trace widths to test printing resolution capabilities at fine pitch layouts. 


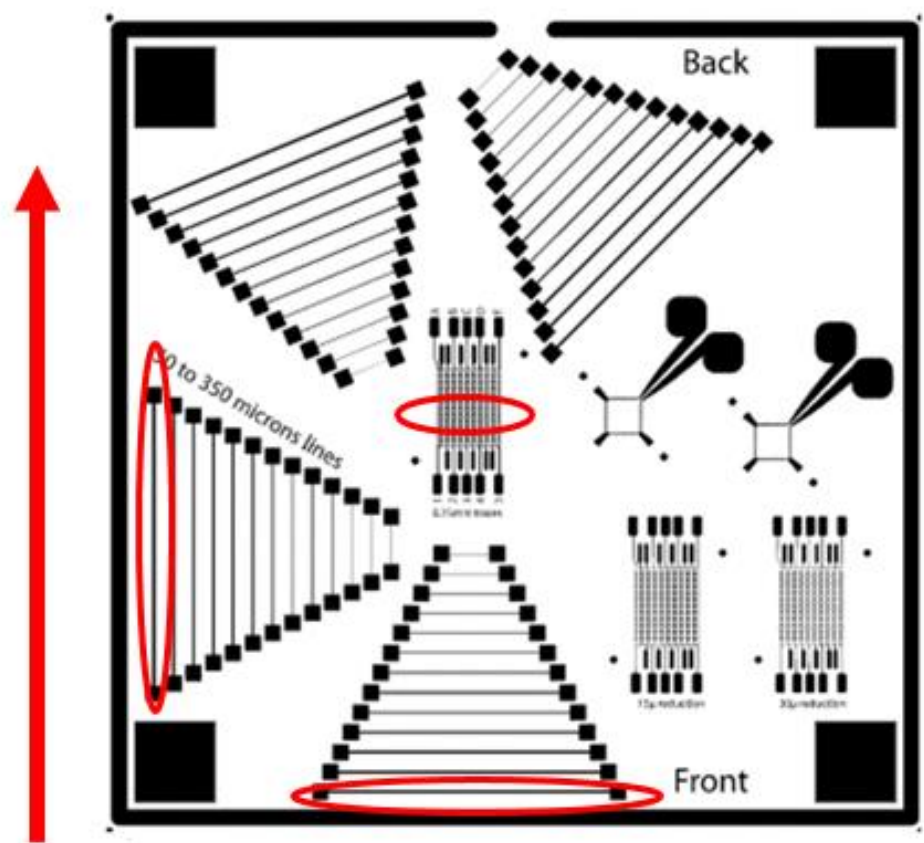

Figure 10. Screen pattern showing the long "ladder lines" and the features used for profilometry measurement and analysis purposes.

\subsubsection{Measurement Methods}

\subsubsection{Resistance}

Resistance was measured using a standard multimeter and two manual probes set at a range of 200 Ohms. Sufficient pressure was applied to avoid contact resistance between the probes and the conductive prints. For measuring and recording the resistance of the ladder lines only two lines from each orientation were measured. The longest line resistance was recorded followed by the shortest line resistance. In the case that the shortest line was not fully connected, the resistance of the first fully conducting line was recorded along with the corresponding line number. For measuring the resistance of the 2 $\mathrm{mm}$ outside line the probes were placed on either side of the line opening in order to record resistance over the entire line. 


\subsubsection{Sheet Resistance}

Sheet resistance is used to measure uniformly thin films, typically in relation to the semiconductor industry. The units of sheet resistance are Ohms/square, allowing the resistance to be calculated for various resistor shapes. For conductive inks sheet resistivity is useful as it gives a theoretical ink property that can then be applied to various print patterns. The sheet resistance provided by the supplier is normalized to a print height of $25 \mu \mathrm{m}$.

Measuring sheet resistance requires the use of a four-point probe (Figure 11). A fourpoint probe is beneficial as it avoids excess contact resistance during measurement. A current is run through the two outer probes and the potential on the inner probes is measured with a voltmeter. For the four-point probe used in this experiment, a geometric factor of 4.53 was applied based on the geometric properties of the circular probe tips. The probe is lowered until electrical connection is made and the corresponding voltage is measured. Using the equation $\mathrm{Rs}=4.53 \mathrm{~V} / \mathrm{I}$ the sheet resistance is calculated. Sheet resistance can be used inline in a manufacturing system for quality assurance verification. 


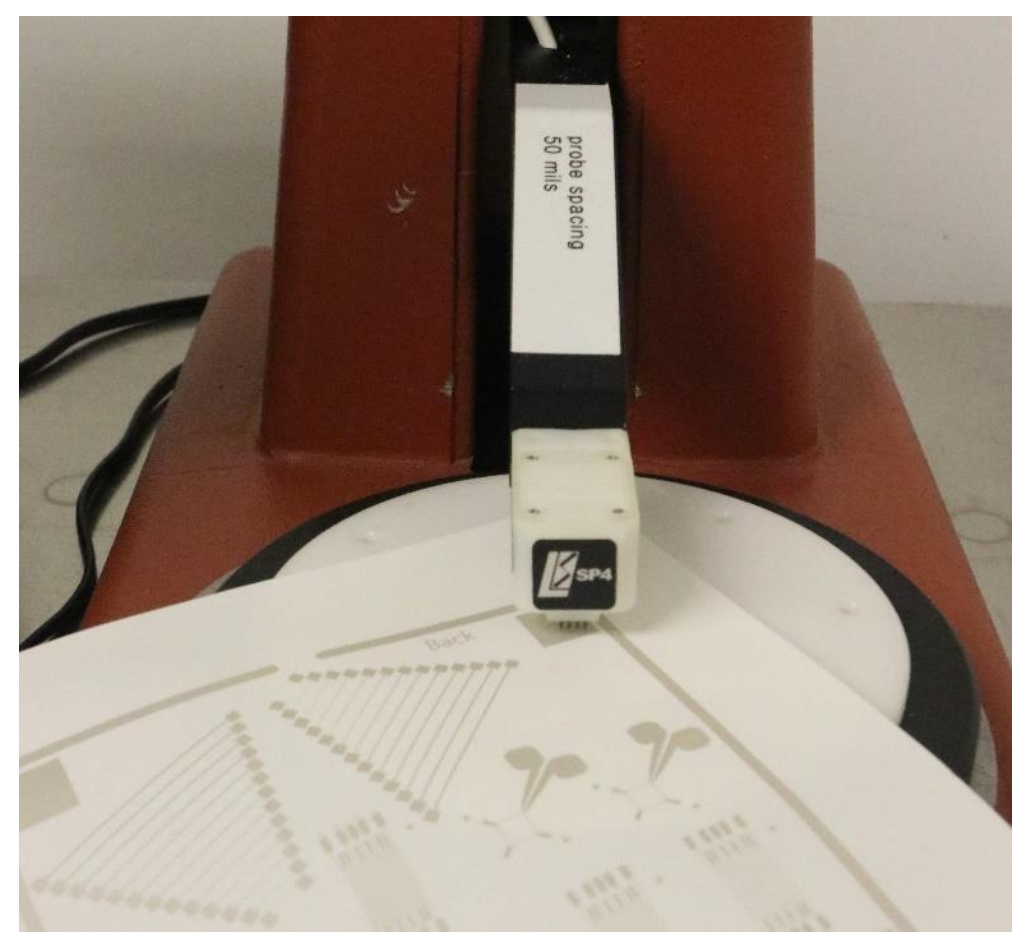

Figure 11. Four-point sheet resistance measurement probe used to measure sheet resistance of thin films. The two outer probes have a current run through them and the results voltage is measured between the inner two probes.

\subsubsection{Print Height}

The measurement of print height, or z-axis thickness was primarily characterized using stylus profilometry. A profilometer is a device used to measure the profile of the surface through various means (Figure 12). This profilometer uses a diamond tip stylus which measures z-axis displacement as it is dragged along the surface. The resolution of the profilometer used is approximately $0.5 \mu \mathrm{m}$, based on previous experimentation and verification. A drag force of 0.8 milligrams was used along with a range of $100 \mu \mathrm{m}$. Measurements of $1 \mathrm{~mm}$ were taken of the longest ladder lines in the orientations parallel and perpendicular to the print direction. Likewise, a $4 \mathrm{~mm}$ long measurement was taken over the center trace footprint to achieve an average trace height value. 


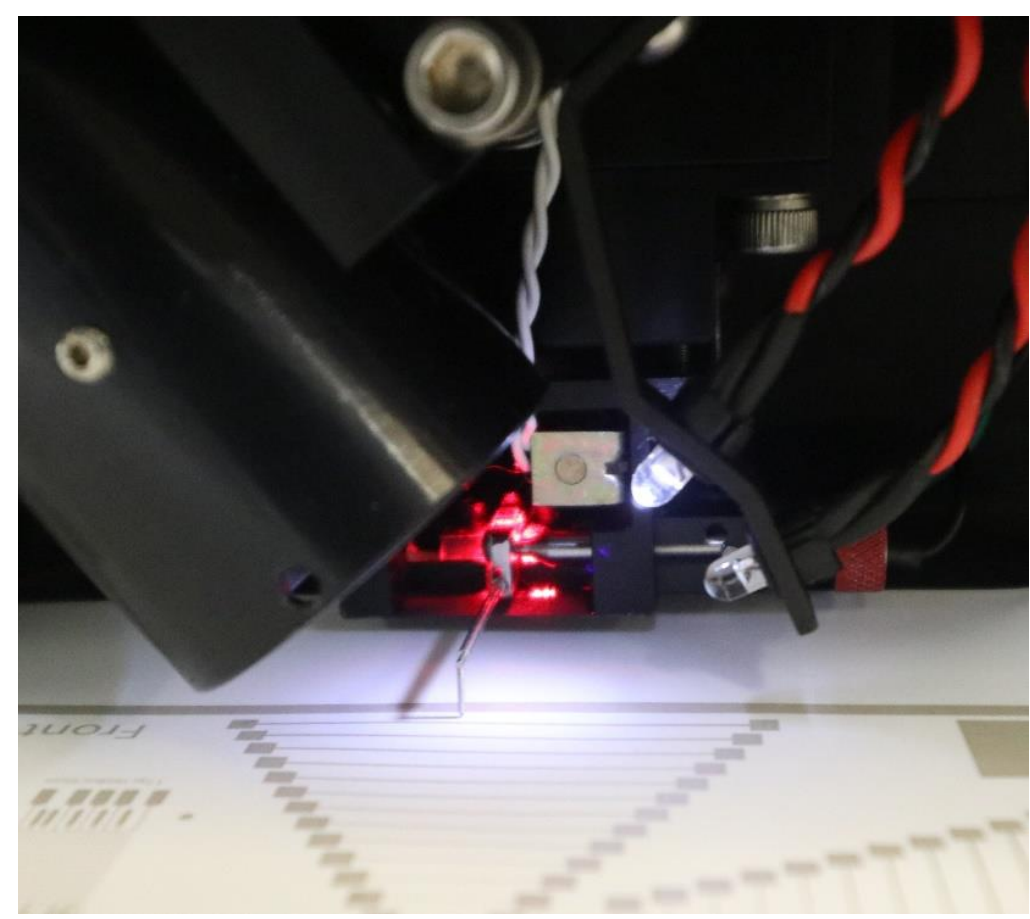

Figure 12. Close up of profilometer cantilever probe used for measuring print height. The resolution of the Ambios profilometer is in the 0-1 micron range.

\subsubsection{Trace Width and Spacing}

The characterization of trace width and spacing was performed by optical microscopy and visual inspection software (Figure 13). Images at 50x magnification were taken of all three PacTech die footprints (normal, $15 \mu \mathrm{m}$ reduction, $30 \mu \mathrm{m}$ reduction). Using the accompanying software and known calibration values lines were drawn to measure trace width, trace length, vertical spacing, and horizontal spacing. These values were recorded in a spreadsheet and later analyzed. 


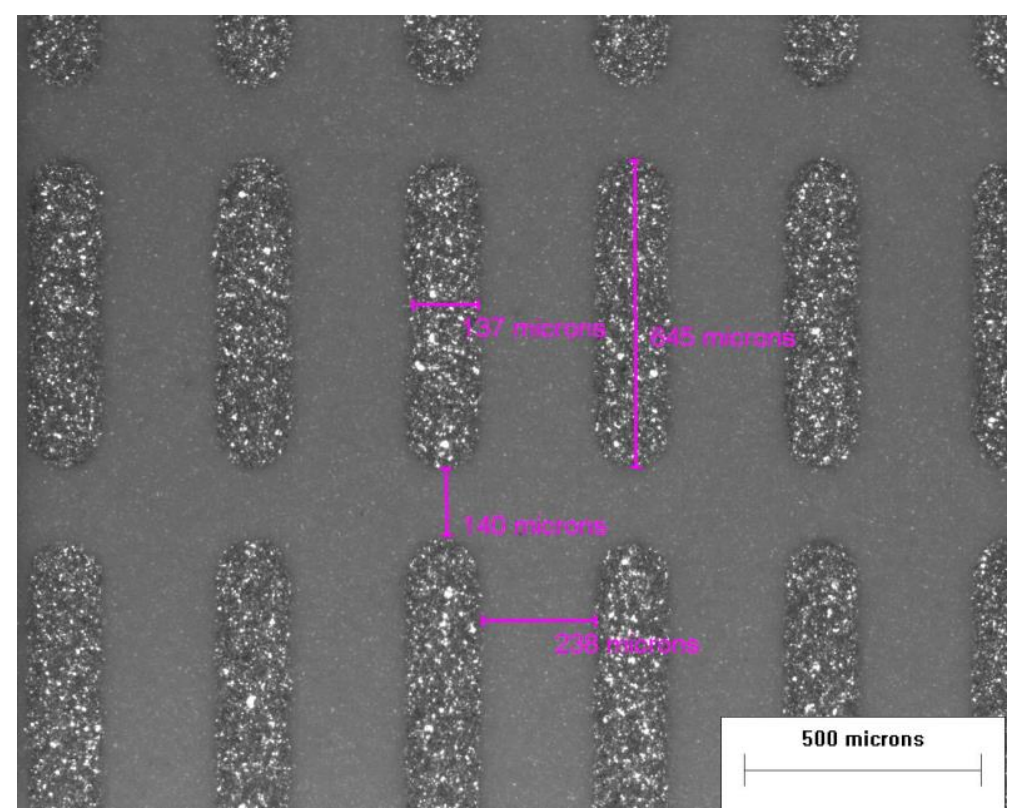

Figure 13. Microscope image of spacing of printed footprint traces for PacTech 2.3 die. The spacing of the traces is critical to daisy chain assembly performance.

\subsubsection{Results and Discussion}

Resistance analysis focused on two primary areas: resistance of individual ladder lines and resistance of the outer trace. A factorial design model was setup in Minitab and the results were statistically analyzed to determine significance of each factor. A Pareto of the significance levels for the outside trace showed the ink, the pressure, and the interaction between ink speed and pressure to be significant (Figure 14). 


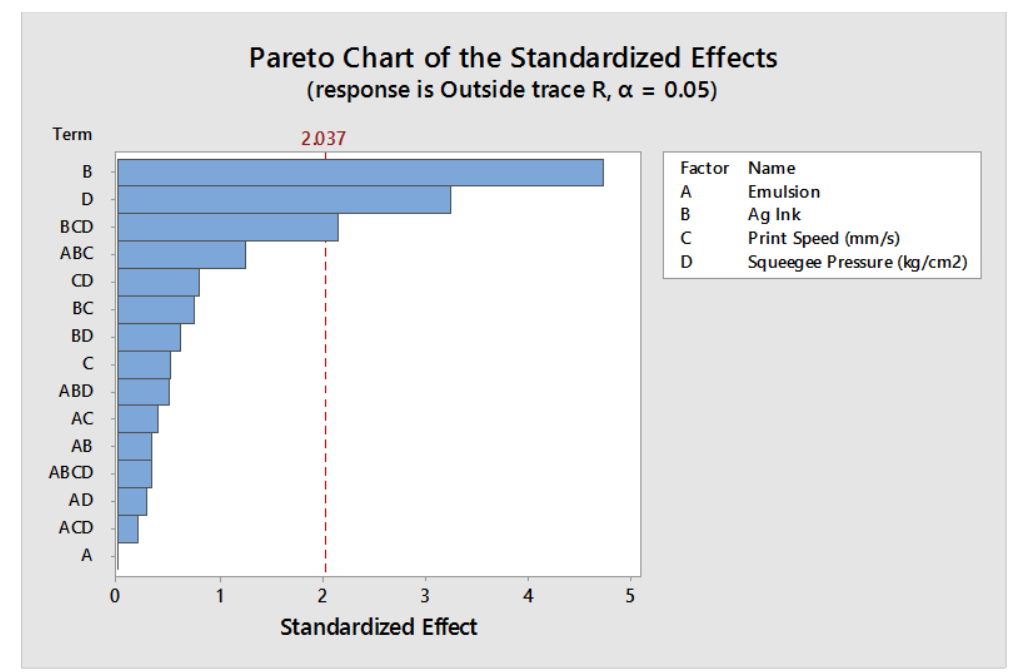

Figure 14. Pareto chart of effects of printing parameters on final resistance values. Print ink, squeegee pressure, and the interaction between ink, print speed, and print pressure were all significant.

The significance of the ink is expected, as one ink is designed to be highly conductive, while the other is designed to be stretchable. The main reason for including it was to have a reference to compare to when analyzing the PE873 ink. The second most significant factor was squeegee or print pressure. Based on the data, a lower print pressure of 2 $\mathrm{kg} / \mathrm{cm}^{2}$ is better for resistance performance (Figure 15). The last significant point, the interaction between ink, speed and pressure, is a third order term and is likely affected by the large ink difference and therefore was not considered for analysis.

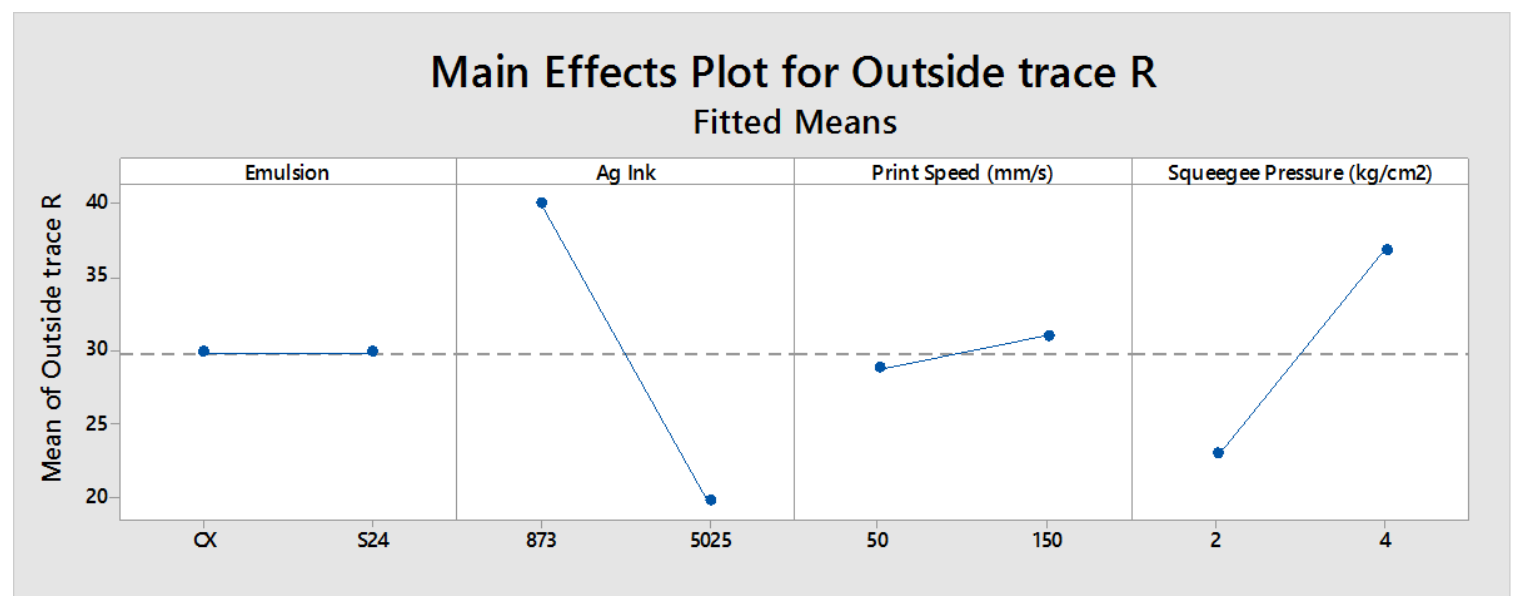

Figure 15. Main effects plot for the exterior pattern $2 \mathrm{~mm}$ trace. Based on the data the squeegee pressure is the primary parameter aside from the ink choice. 
The longest lines "ladder line" features were also analyzed using similar methods (Figure 10). These lines had the potential to perform different in printing conditions due to thinner dimensions. Looking at the analysis, both ink and pressure are significant again, however emulsion has also shown up as significant (Figure 16). This is explained by the thickness differences of the emulsions, with S24 leading to thinner, taller features, and $\mathrm{CP}$ leading to shorter, wider features. (Further discussion on this explained in the profilometry section).
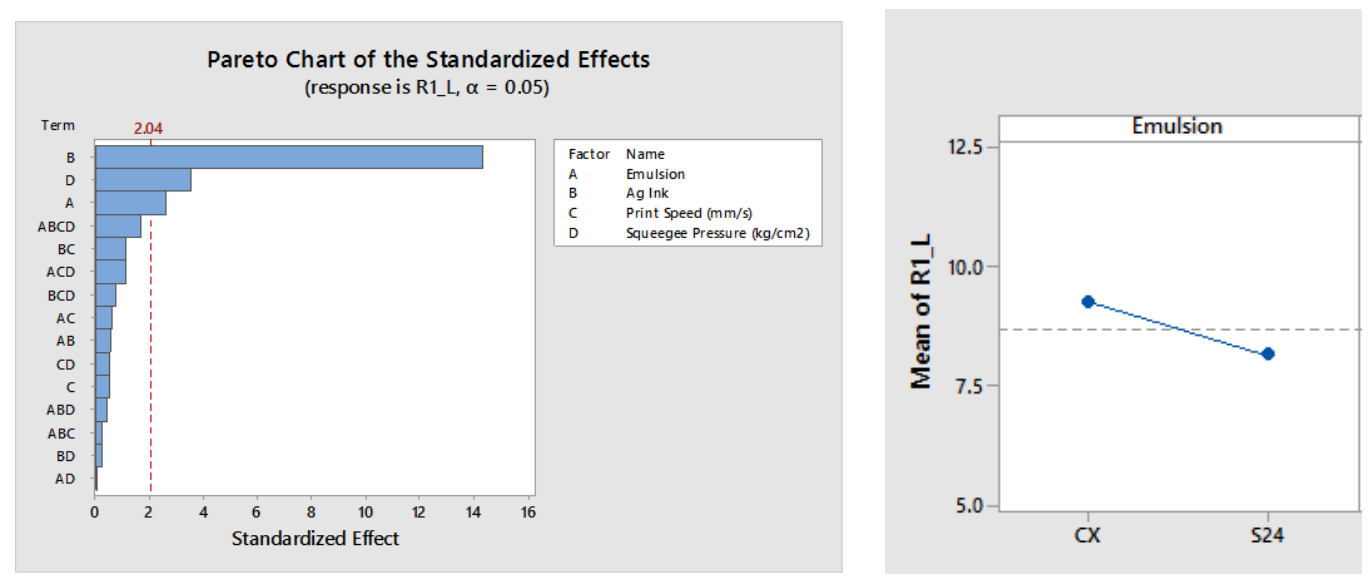

Figure 16. Pareto chart of significance levels of longest trace. 15b. Main effects plot of emulsion. Based on the resistance data it was determined that a lower print pressure and lower print speed should be used.

For sheet resistance, each of the four squares in the corners of the print pattern were measured using a 4-point probe with a current of $100 \mathrm{~mA}$. Gathering the data proved difficult with many of the samples producing different sheet resistance values depending on the orientation of the 4-point probe. The mean sheet resistance of the samples resulted in a value of roughly $200 \mathrm{~m} \Omega /$ square, however when doing hand calculations using the measured dimensions of the print, the theoretical sheet resistance came out to be around $75 \mathrm{~m} \Omega /$ square. After further analysis using profilometry to measure the profile of the square pads, it was determined that the pads contained large amounts of scooping, and 
therefore the printed ink was not uniform in thickness, which is essential when using a 4point probe (Figure 17). Therefore, the analysis going forward will focus on simply resistance as a determination factor and calculate sheet resistance, comparing it to the manufacturer's specified values.

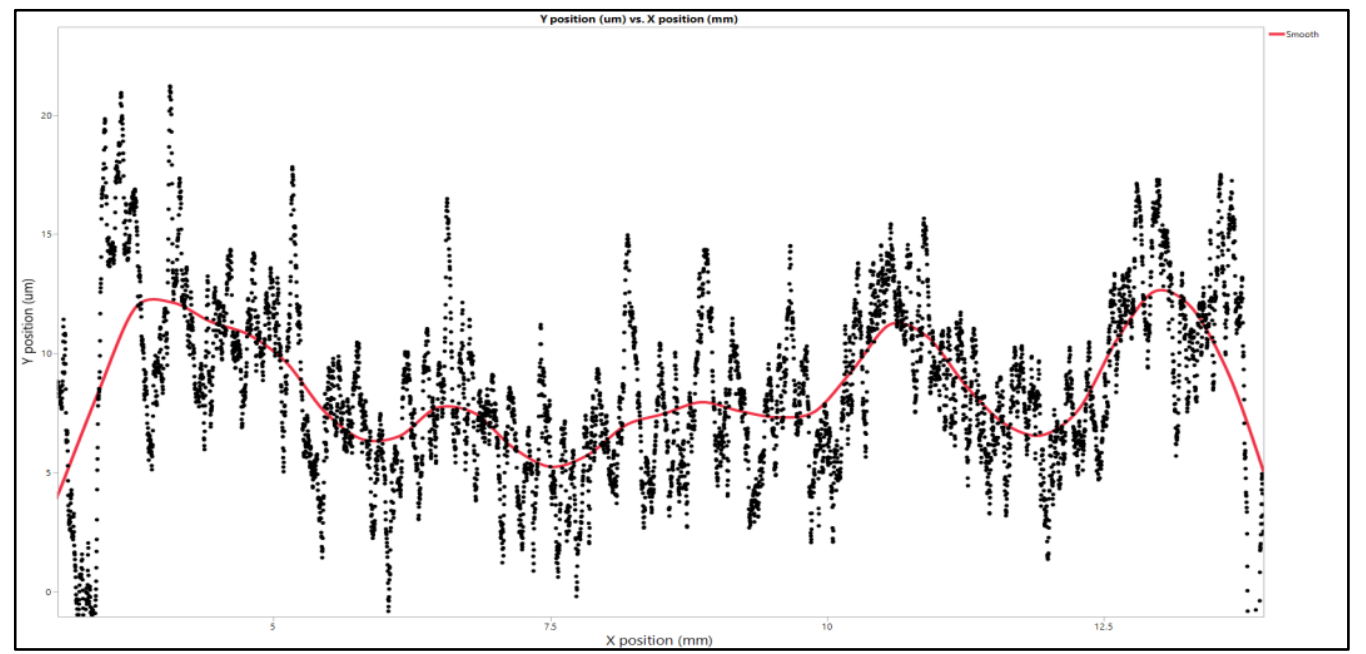

Figure 17. Profile of square pad showing scooping outline. Based on the un-uniformity it is unlikely that sheet resistance using the 4-point probe is unviable.

A large factor in determining quality prints is print height. The resistance is directly proportional to the amount of silver in the trace, therefore achieving the best possible print height is essential to producing quality prints. Using the profilometer, the print heights of each sample were measured along parallel and perpendicular traces, as well as along the center of the die footprint (Figure 10, Figure 18). 


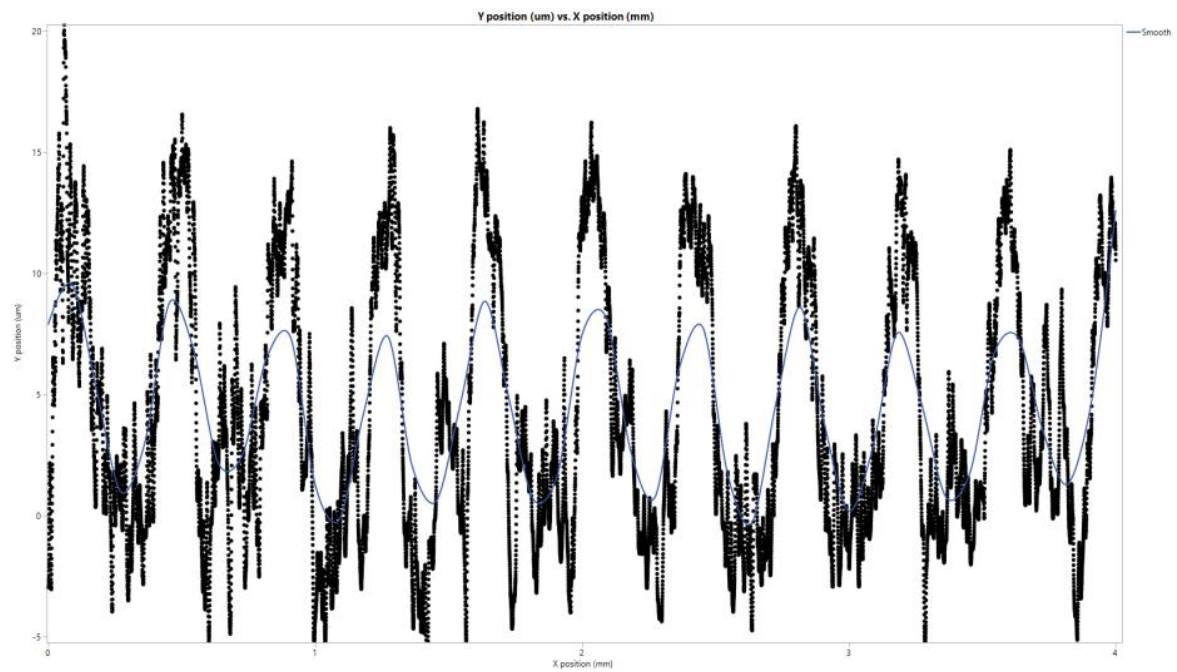

Figure 18. Profile of inner traces of PacTech die footprint. The average height of the traces was estimated and used for analysis.

Initial testing produced much noise in the profilometer data, therefore additional testing was conducted using just the TPU (no liner), taped down to a glass slide. Tests showed the TPU to have an overall roughness ranging from 6-8 $\mu \mathrm{m}$. This became a challenge when analyzing several of the poorer quality prints which only had trace heights of around $8 \mu \mathrm{m}$ (Figure 19). Results showed distinct difference in the height of the two inks, with the 5025 having print heights around $20 \mu \mathrm{m}$ on average, and the PE873 having heights around 10-12 $\mu \mathrm{m}$. Analysis was conducted and the results look similar to the resistance significance (Figure 20). 


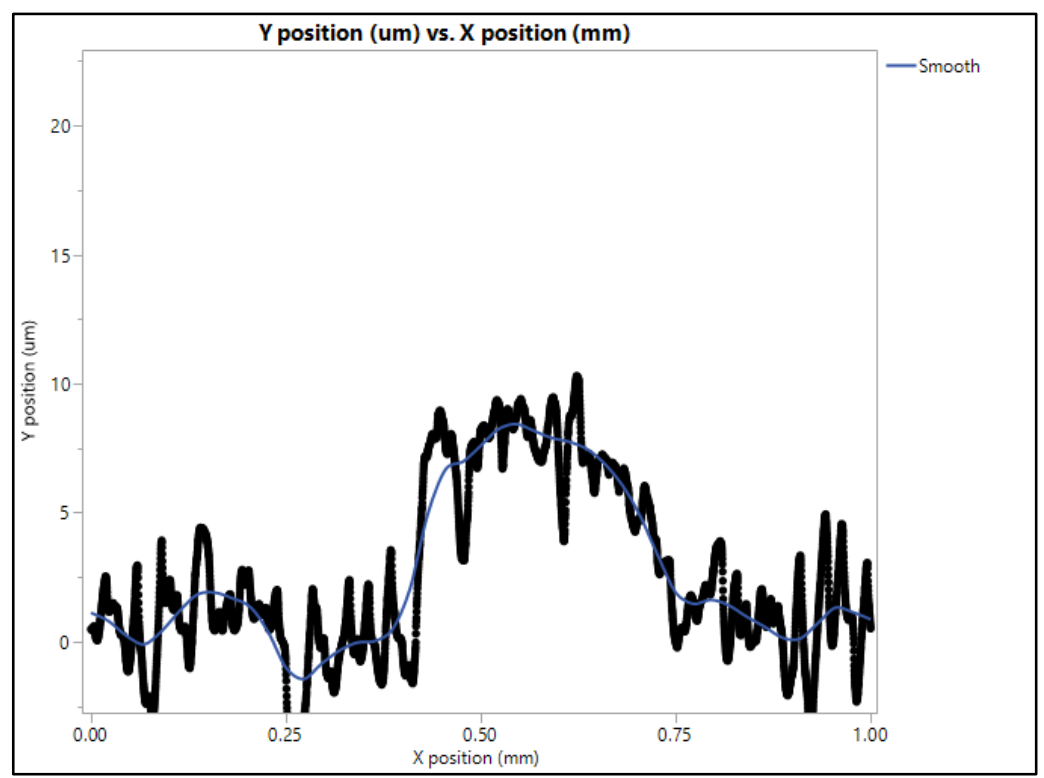

Figure 19. Side profile of PE873 ink printed on TPU. The large surface roughness of the TPU made it challenging for verifying print height with certain parameters leading to a print roughly $8 \mu \mathrm{m}$ tall and $0.35 \mathrm{~mm}$ wide.

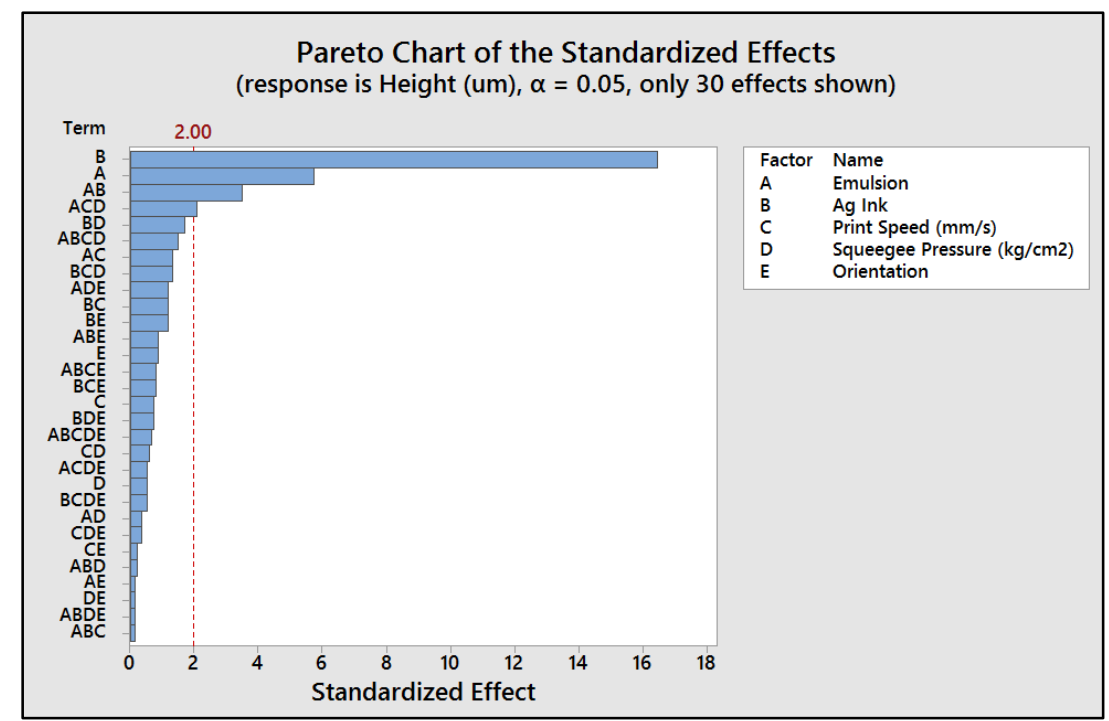

Figure 20. Pareto chart results for significance of PE873 printed traces in relation to overall print height.

Observations of the trace widths and spacing for all 144 samples showed no sign of bleeding or expansion in the prints. Analysis showed prints with the S24 emulsion to have slightly thinner printed features at the $150 \mu \mathrm{m}$ thickness. This is due to the geometry 
of the S24 emulsion being thicker. The resulting print, therefore, has a higher height to width aspect ratio, which in turn produces thicker prints with better performance.

\subsubsection{Photonic Curing}

After much discussion about curing of the conductive inks, it was suggested that perhaps the samples had not fully cured and thus additional curing may be of value. Likewise, the photonic curing system had yet to be used in the experiment thus far, so it was decided to run the samples through the NovaCentrix curing system in order to observe any possible changes. Settings were based on previously successful runs for 5025 ink on PET. After several adjustments, a final voltage of $380 \mathrm{~V}$ was used following a similar profile used on the 5025. Results did show a decrease in resistance, thus proving the point that the samples had not fully cured originally (Table 2). It is important to not over cure though, as over curing generates too much energy in the samples and will burn the traces off the TPU (Figure 21).

Table 2. Differences in resistance values after Photonic Curing.

\begin{tabular}{|c|c|c|}
\hline Sample \# & Outer Trace \% Difference & Average Trace \% Difference \\
\hline 13 & -6.2 & -16.6 \\
\hline 15 & -36.4 & -48.9 \\
\hline 17 & -26.1 & -39.2 \\
\hline 19 & -45.2 & -47.2 \\
\hline 41 & -71.9 & -35.2 \\
\hline 43 & -145.7 & -50.1 \\
\hline 44 & -48.4 & -28.2 \\
\hline 45 & -38.5 & -39.3 \\
\hline
\end{tabular}



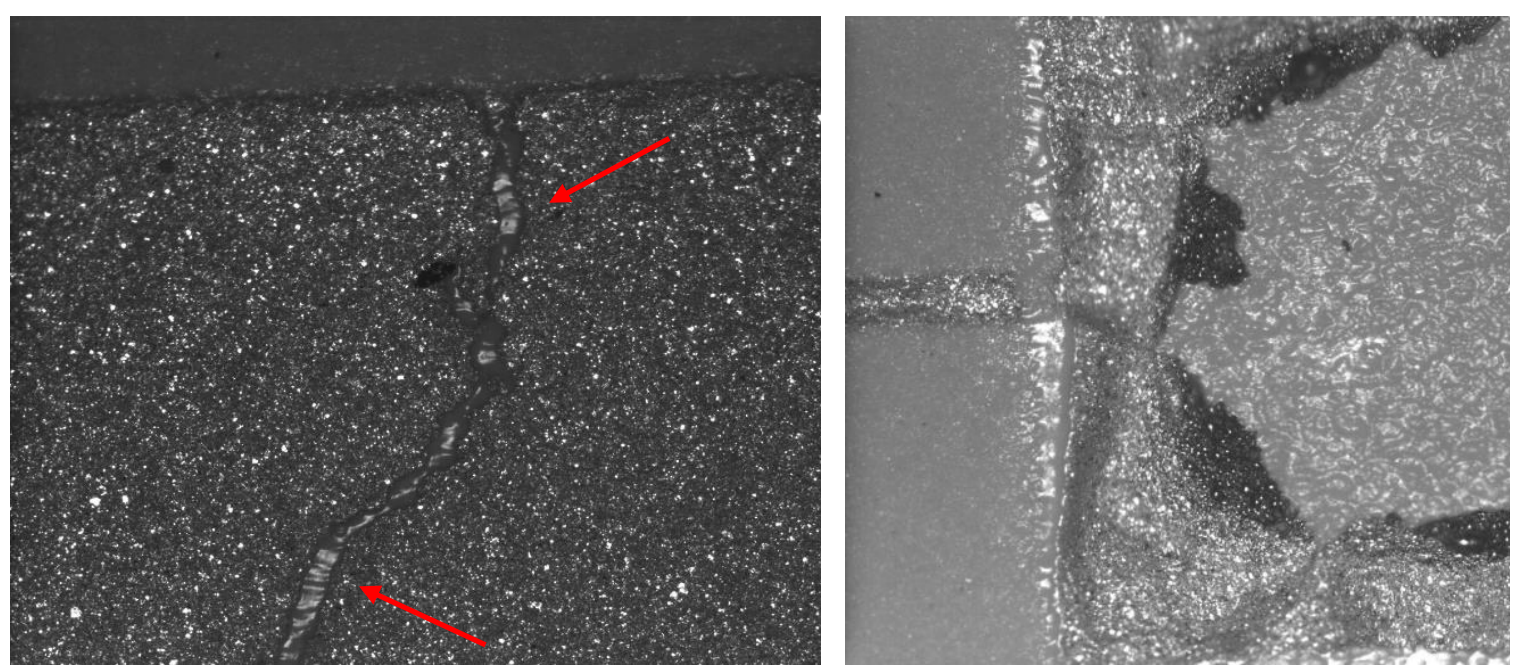

Figure 21. Sample of over cured print using photonic curing. Lift off and separation are common failure methods.

\subsubsection{Preliminary Reliability Tests}

First preliminary tests for reliability were conducted based on the provided ASTM testing methods. This involved a 180 degree "crease test" (ASTM F2749-15), a tape test for adhesion (F1842-15), and an abrasion test (D5264-11). The crease test only used one sample and the sample was creased with 10 cycles, perpendicular to the ladder lines, with only the TPU (no liner) as a supporting material. After these 10 cycles the results were measured in terms of the resistance increase of each line (Figure 22). Next a tape test was conducted on the four squares in each corner of the pattern, with each square getting sectioned into six columns and six rows. Of the five samples tested, all samples performed at a level 5 result, with no removal of any ink material. Lastly the abrasion test showed good results as well with little increase in resistance values after testing. 


\begin{tabular}{|r|r|r|r|}
\hline \multicolumn{1}{|c|}{ Trace \# } & \multicolumn{1}{c|}{ Pre R } & \multicolumn{1}{l|}{ Post R } & \% Increase \\
\hline 1 (shortest) & 11.6 & 27 & $133 \%$ \\
\hline 2 & 13.2 & 29.2 & $121 \%$ \\
\hline 3 & 12.9 & 27.2 & $111 \%$ \\
\hline 4 & 13.4 & 35.4 & $164 \%$ \\
\hline 5 & 14.1 & 58 & $311 \%$ \\
\hline 6 & 15.2 & 59.5 & $291 \%$ \\
\hline 7 & 15 & 58.4 & $289 \%$ \\
\hline 8 & 16.8 & 63.4 & $277 \%$ \\
\hline 9 & 15.9 & 67 & $321 \%$ \\
\hline 10 & 17 & 126 & $641 \%$ \\
\hline 11 & 17.5 & 80 & $357 \%$ \\
\hline 12 & 21.7 & 79 & $264 \%$ \\
\hline 13 & 33.3 & 218 & $555 \%$ \\
\hline & & & \\
\hline
\end{tabular}

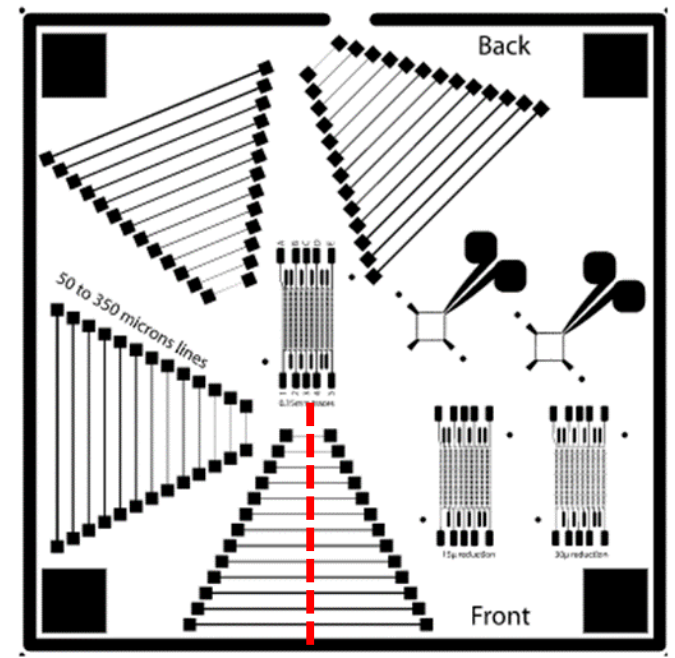

Figure 22a. Results from after 10 cycles of crease testing. 22b. Pattern sample showing location of crease on print.

\subsection{Print Phase 2}

\subsubsection{Objective}

The objectives of the second phase printing development experiments were to 1)

determine the optimal curing method available at Cal Poly for curing of the samples, and 2) use the results of the phase one print experiment to refine the convection oven curing process to match the results of the box oven. Previous printing experiments had provided the optimal parameters for use on the DEK printer, and this experiment sought to achieve the best resistance and reliability performance based on the three curing methods: box oven, convection oven, and photonic curing system.

\subsubsection{Experiment Design}

Based on the results from the phase two print experiment the best printing parameters for use with the PE873 ink include a print pressure of $2 \mathrm{~kg} / \mathrm{cm}^{2}$, a print speed of $50 \mathrm{~mm} / \mathrm{s}$, 
and the use of the S24 emulsion. All other printing variables in this experiment were consistent with the previous experiment.

In terms of curing parameters for the three curing methods, the following parameters were decided after several initial tests. Box oven: cure for 30 minutes at $135^{\circ} \mathrm{C}$ using the same Espec box oven previously used. Convection oven: using the Heller 5 zone conveyor convection oven at Cal Poly set the conveyor speed to $50 \mathrm{~cm} / \mathrm{min}$ with $150^{\circ} \mathrm{C}$ temperature setting in all 4 main zones and $80^{\circ} \mathrm{C}$ in the first zone. Photonic Curing: with the NovaCentrix Pulseforge 1200 use a photonic energy profile that includes a max power of 380 volts, with 5 micropulses following a 500/250 milliseconds and 150/250 milliseconds on/off pattern. Additional details on the development and arrival of these parameters below. A total of 45 samples were produced, with 15 samples cured by each method. The goal was to take each sample group and test the overall resistance, followed by at least 5 samples each for tape testing, crease testing, and preliminary wash/dry cycle testing (Figure 23).

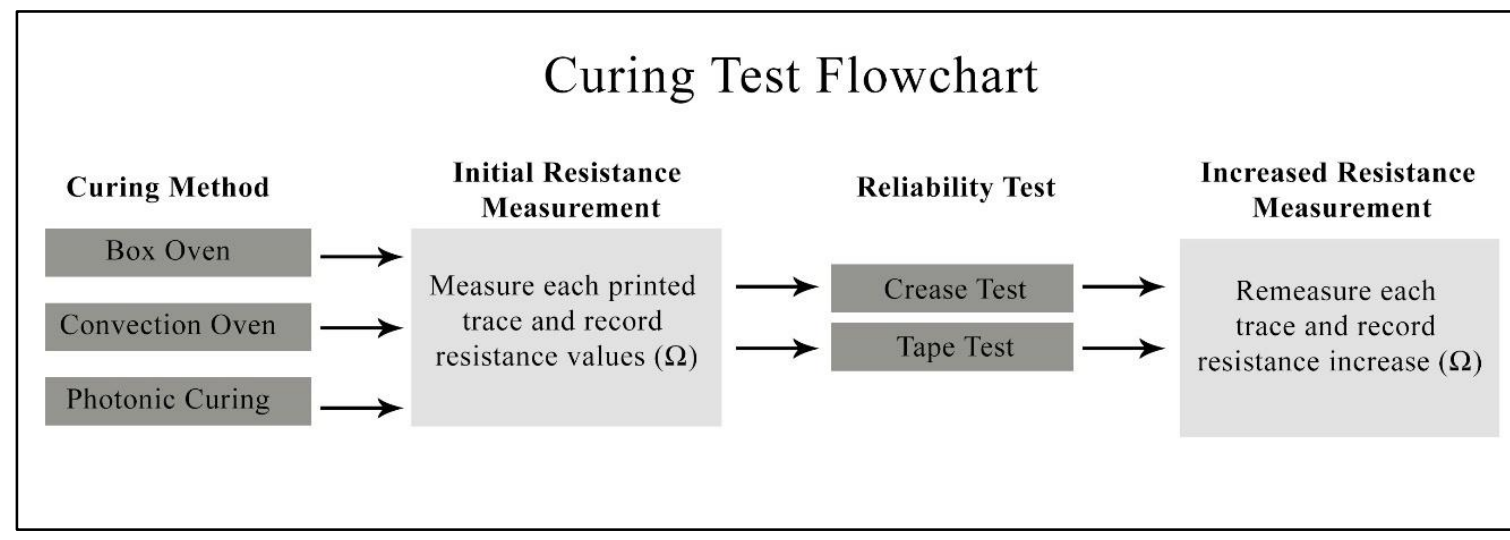

Figure 23. Flowchart of curing test for Phase 2 print experiment. Based on the initial resistance measurements and the reliability test results, one curing methods was selected for moving forward with the project. 


\subsubsection{Measurement Methods}

Resistance was measured using a standard multimeter and two manual probes set at a range of $200 \mathrm{Ohms}$. Sufficient pressure was applied to avoid contact resistance between the probes and the conductive prints. For measuring and recording the resistance of the ladder lines only two lines from each orientation were measured. The longest line resistance was recorded $(350 \mu \mathrm{m})$ followed by the third shortest line $(100 \mu \mathrm{m})$ resistance. This is due to multiple prints not being conductive on the 50 and $75 \mu \mathrm{m}$ thick lines. Crease testing was performed following ASTM F2749-15 standards. The 15 samples (5 for each curing method) were cut in half and heat pressed onto an Under Armour/Lycra Wickaway Blend fabric for 15 seconds at $120^{\circ} \mathrm{C}$. The fabric was attached to simulate the bending radii that a sample might realistically experience during use. Each sample was creased 10 times using a constant rolling force (Figure 24). The change in resistance value was measured for the $100 \mu \mathrm{m}$ and $350 \mu \mathrm{m}$ lines and recorded.
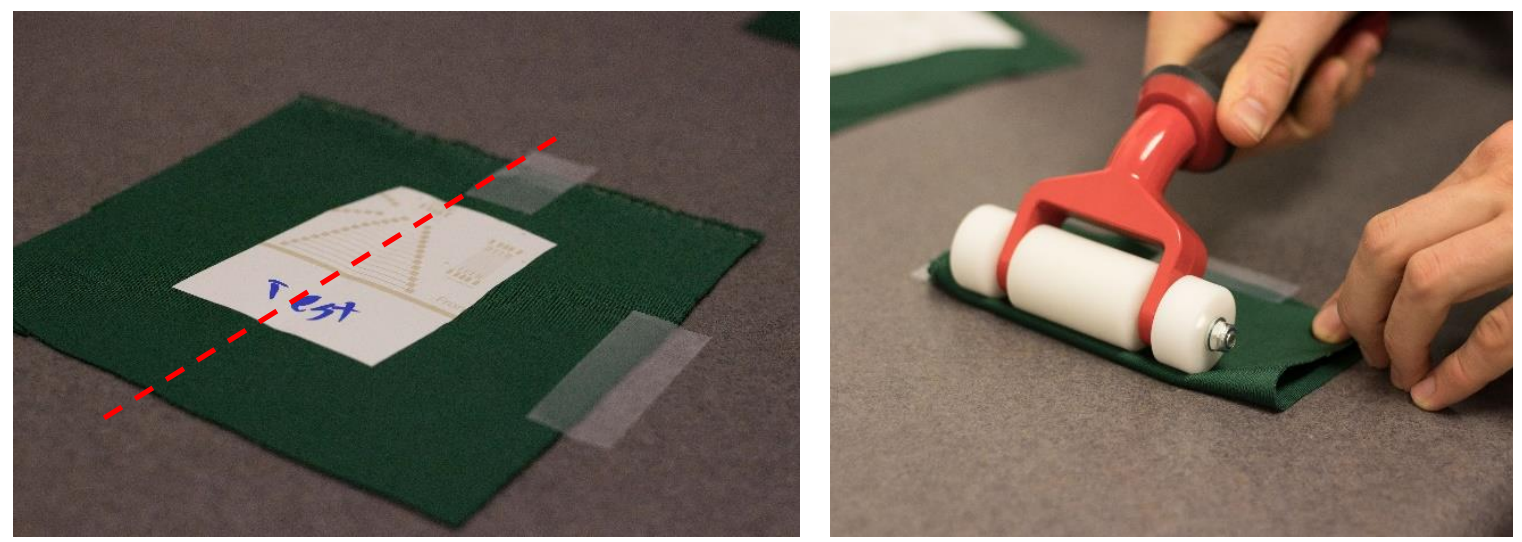

Figure 24. Samples were heat pressed onto fabric and creased 10 times along the center of the ladder lines. Samples were creased using a Delrin carpet roller to ensure even pressure throughout the crease.

The tape test was performed per ASTM F1842-15 standards, using a 1 inch wide tape from $3 \mathrm{M}$ as specified in the standard. The $10 \times 10 \mathrm{~mm}$ squares in the corners of each 
sample were used for testing, with a total of 30 samples tested per curing method. Results were determined using the adhesion classification test results in the ASTM standard. In order to gauge a better idea of how well the samples would perform in the presence of water, a preliminary wash test experiment was conducted. Samples adhered to fabric were placed in a bowl of water with laundry detergent at a ratio similar to that of a standard laundry load. The samples were then vigorously mixed for 30 seconds (Figure 25). After mixing, the samples were rinsed in a separate bowl of room temperature water and then air dried for two hours. After drying, the resistance values of the lines were recorded. It is important to note that these samples had no form of conformal coating over the exposed ink, therefore results may vary compared to final assembled devices.

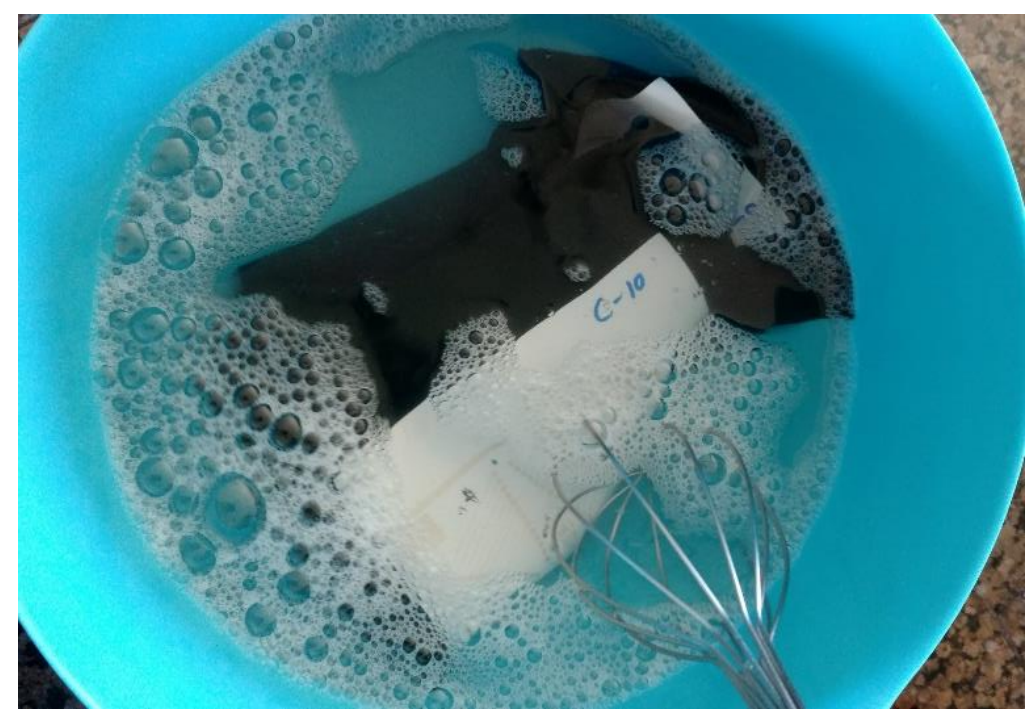

Figure 25. Samples were washed in detergent for 30 seconds then air dried. A whisk was used to simulate the spinning of a normal washing machine.

\subsubsection{Results and Discussion}

\subsubsection{Resistance}

The initial resistance measurements from each of the tests were used to determine which test proved best moving forward in the experiment. The three different methods were 
compared and ultimately one method was suggested for use. Results showed a variation in lowest resistance among the curing methods, with box oven proving to have the best resistance performance overall at an average resistance value of 9.9 Ohms (Figure 26).

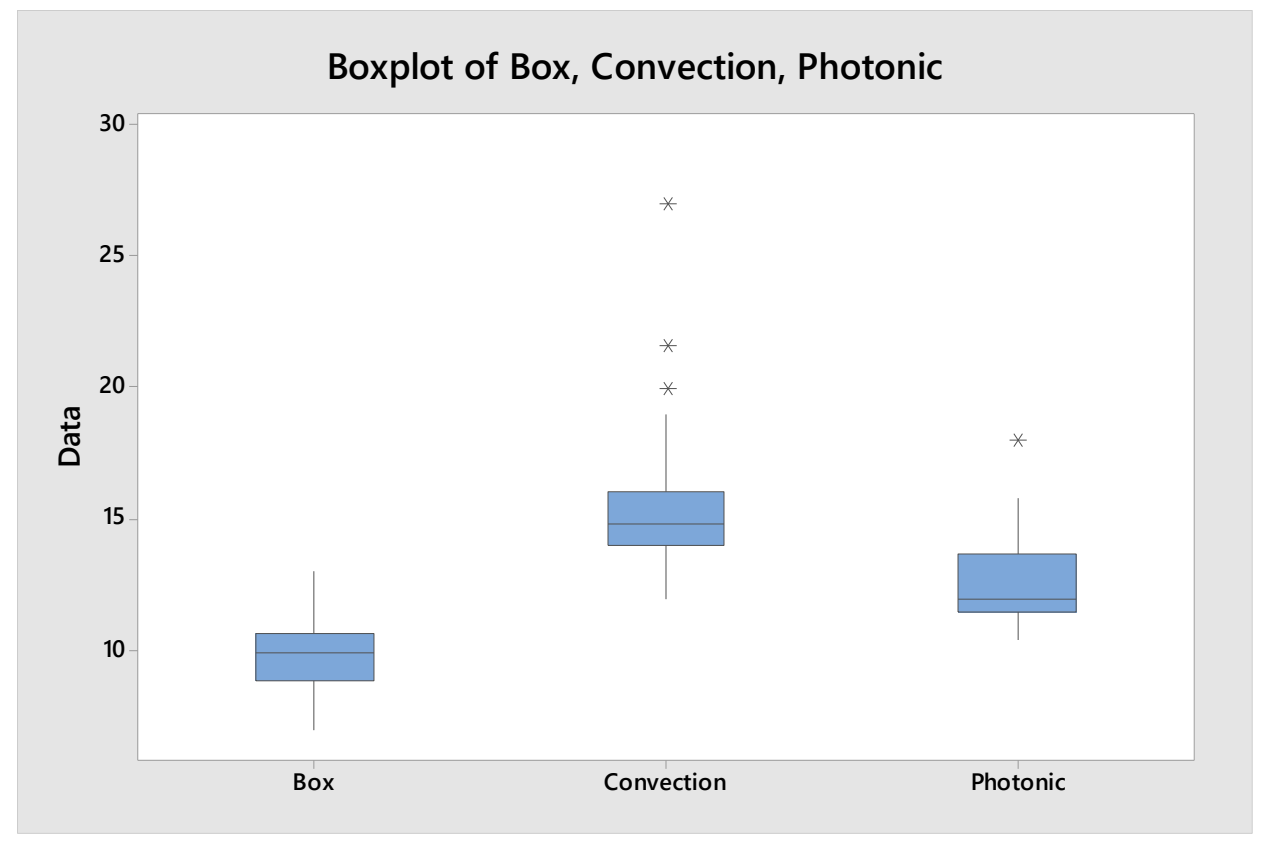

Figure 26. Boxplot of resistance results $(\Omega)$ for each curing method used.

The data also shows parallel to print direction as having the best performance (Figure 27). This is consistent with known expectations, as perpendicular lines tend to have greater amounts of scooping of the ink during squeegee pass. The standard deviation in the line resistances for box oven, convection oven, and photonic curing were 1.1, 1.2, and 1.0 Ohms respectively. 


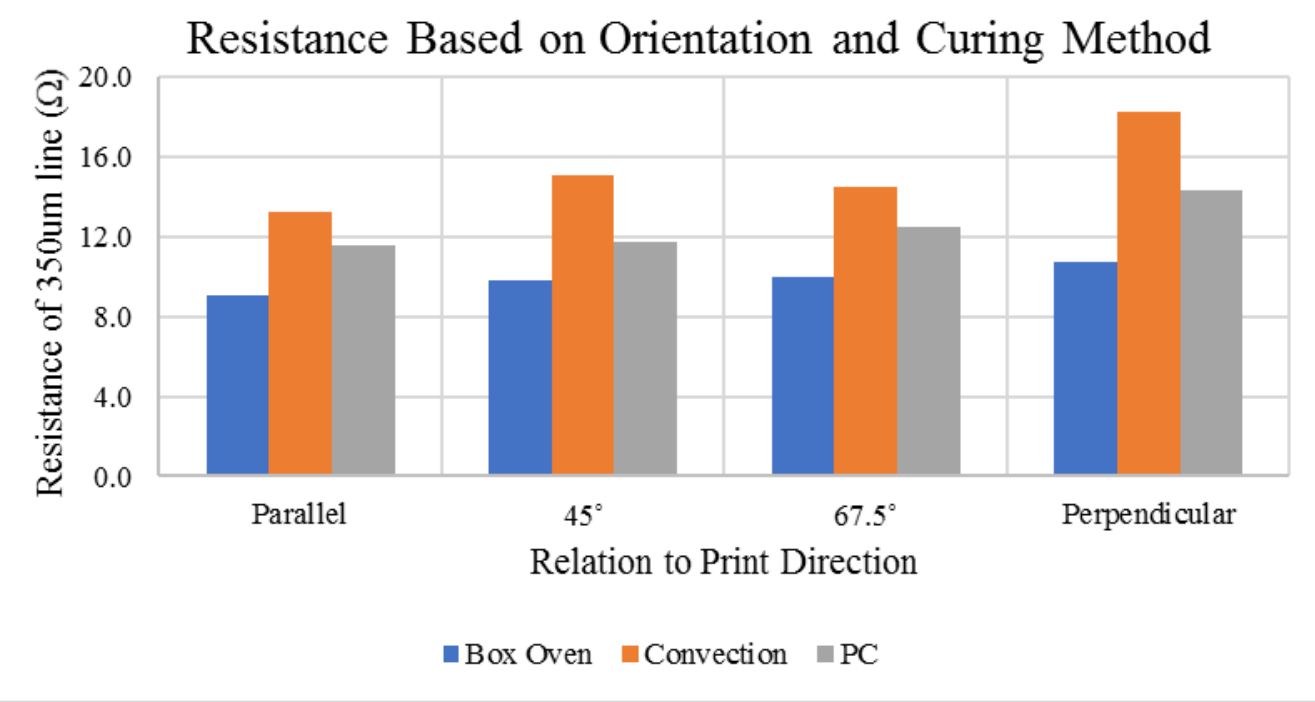

Figure 27. Chart comparing resistance results based on print orientation and curing method.

It is important to note that even though the box oven proved to be the best curing method at this point in time this does not guarantee that the box oven is the best method for curing the samples. For this test, little experimentation was performed with the convection or photonic curing oven, therefore it is possible that neither of those ovens had been fully optimized for the curing process at this time. Later tests discuss the results of additional oven optimization.

\subsubsection{Reliability Tests: Crease Test}

After all 45 samples had been cured, the samples were divided into groups for performing reliability tests. For the crease test, 5 samples from each method were cut in half prior to adhering to the Poly/Lycra blend fabric. The resistance results for before and after the 10 cycles were measured for both the $350 \mu \mathrm{m}$ and $100 \mu \mathrm{m}$ lines (Table 3). 
Table 3. Phase 2 Crease Test Resistance Results.

\begin{tabular}{|c|c|c|c|c|c|c|c|}
\hline & & \multicolumn{2}{|c|}{$\mathrm{R}$ post 10 cycles $(\Omega)$} & \multicolumn{2}{|c|}{$\%$ Increase } & \multicolumn{2}{|c|}{24 Hr Relaxation } \\
\hline & & Average & StdDev & Average & StdDev & Average & StdDev \\
\hline Longest & \multirow{2}{*}{ Photonic } & 87 & 24 & $425 \%$ & $138 \%$ & $-33 \%$ & $14 \%$ \\
\hline Shortest (100um) & & 546 & 608 & $2355 \%$ & $2828 \%$ & $-48 \%$ & $20 \%$ \\
\hline Longest & \multirow{2}{*}{ Box Oven } & 28 & 11 & $77 \%$ & $32 \%$ & $-31 \%$ & $12 \%$ \\
\hline Shortest (100um) & & 34 & 22 & $164 \%$ & $71 \%$ & $-36 \%$ & $27 \%$ \\
\hline Longest & \multirow{2}{*}{ Convection } & 130 & 61 & $489 \%$ & $342 \%$ & $-52 \%$ & $11 \%$ \\
\hline Shortest (100um) & & 527 & 506 & $1843 \%$ & $2069 \%$ & $-54 \%$ & $11 \%$ \\
\hline
\end{tabular}

The results show a distinct performance difference between in both the length of the lines being tested and the method by which they were cured. The $350 \mu \mathrm{m}$ lines on the box oven samples performed the best, increasing resistance by $77 \%$ on average, followed by the $100 \mu \mathrm{m}$ box oven lines. Both the photonic cured samples and convection reflow oven samples saw significant increases in resistance values, along with large variations in those increases. This performance is likely explained by the fact that the box oven samples were the only samples which had been fully cured post printing. For the other samples, it is possible that voids had formed within the conductive ink traces, thus leading to worse performance upon the application of stresses.

The last column Table 3, shows how the resistance values performed 24 hours after the crease test. It was observed during testing that samples that had sat for long periods of time showed improved resistances than those that had been measured directly after creasing. Therefore all 45 samples were remeasured 24 hours after the creasing test to observe if there was any relaxation factor that occurred within the ink traces. All three curing methods showed noticeable levels of resistance decrease, with the convection oven curing recovering the most. While further investigation is needed to verify the reasoning behind this performance, the theory is that as the ink and substrate are relaxing post 
creasing and more of the silver particles in the trace are beginning to reconnect and contact each other, which in turn increases the conductivity.

\subsubsection{Reliability Tests: Tape Test}

The tape test was performed using the specifications from the ASTM standard and all samples passed with a rating of 5, per the ASTM rating scale (Figure 28). This rating is defined as "The edges of the cuts are completely smooth; none of the squares of the lattice is detached" (Appendix B). After conducting a total of 90 tape tests across the 3 curing methods it was clear that proper adhesion had been achieved regardless of curing method. Due to the large surface roughness of the TPU substrate it is hypothesized that adhesion will not be an issue when dealing with printing, assuming the ink is neither under-cured, which would result in liftoff, nor over-cured, which would result in burning off the ink.

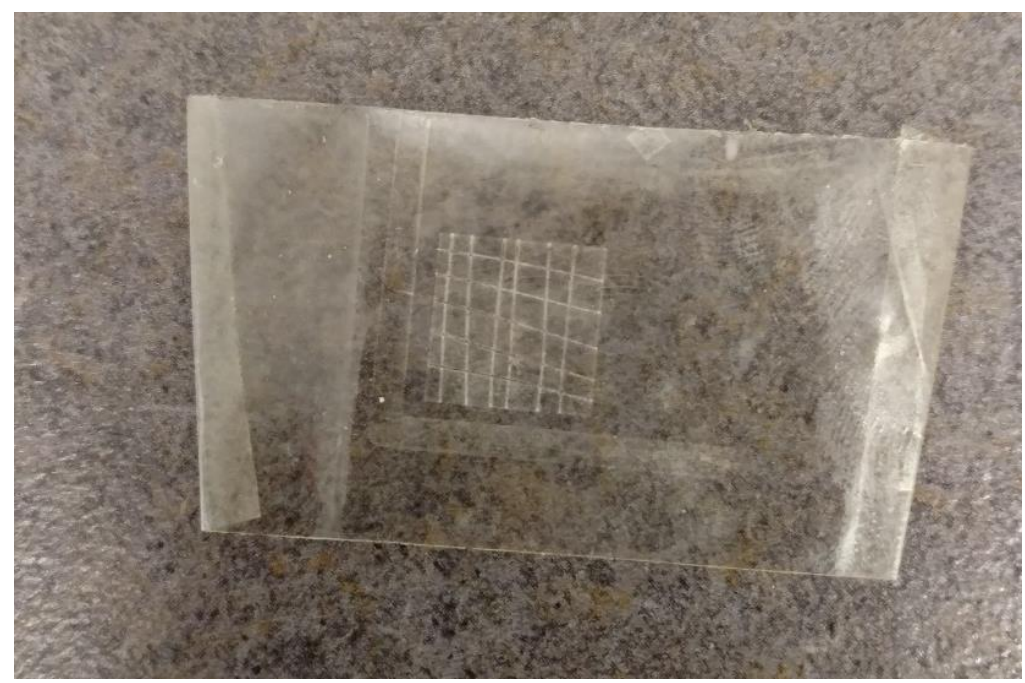

Figure 28. Tape test results showing no peeling of ink. Lines shown are material removed due to scoring with a razor blade.

\subsubsection{Reliability Tests: Wash Test}

Given the preliminary nature of the experiment, the results will be further investigated once more exhaustive tests have been conducted. After testing the results appeared 
similar to those of previous reliability tests with the box oven samples showing better performance compared to those of the photonic curing and convection oven (Table 4). Table 4. Wash Test Resistance Increase Results.

\begin{tabular}{|c|c|c|c|c|}
\hline & $\begin{array}{c}\text { Increase in } \mathrm{R} \\
{[\Omega](350 \mu \mathrm{m})}\end{array}$ & $\begin{array}{c}\text { Increase in } \mathrm{R} \\
{[\Omega](100 \mu \mathrm{m})}\end{array}$ & $\begin{array}{c}\% \text { Change } \\
350 \mu \mathrm{m}\end{array}$ & $\begin{array}{c}\% \text { Change } \\
100 \mu \mathrm{m}\end{array}$ \\
\hline Photonic & 89.8 & 68.2 & $193 \%$ & $56 \%$ \\
\hline Convection & 57.4 & 67.6 & $161 \%$ & $79 \%$ \\
\hline Box Oven & 8.4 & 3.4 & $56 \%$ & $21 \%$ \\
\hline
\end{tabular}

Unlike the previous tests however, the wash test had greater increase in resistance for the $350 \mu \mathrm{m}$ lines when compared to the $100 \mu \mathrm{m}$ lines. This observation is likely due to the interaction between the water and the ink, which, given the larger surface area of the 350 $\mu \mathrm{m}$ lines, would make sense for the interaction to have a greater effect. It is hypothesized that further wash tests would not prove to be as destructive to the samples based on the use of a conformal coating to prevent environmental interference with the inks.

\subsection{Print Phase 3}

\subsubsection{Objective}

Upon discussion and investigation of the previous printing resistance results it was hypothesized that perhaps the convection oven and photonically cured samples did not perform as well because they had not been fully cured. Therefore, additional testing was performed on tuning the convection oven to achieve similar results to the box oven. 


\subsubsection{Design and Methods}

The primary variables for adjusting the convection oven are speed of the conveyor belt and top and bottom temperatures of the 5 oven zones. For adjusting of the tuning, a mixture of speeds and temperature ramp levels were tested and the ensuing resistance values measured. Ultimately a temperature profile of 80-130-150-150-150 was chosen along with a speed of $30 \mathrm{~cm} / \mathrm{min}$. These parameters were designed to mimic the same amount of time at peak temperature previously used, just with a slower ramp rate. This slower ramp rate was chosen due to some levels of warping and damage to the substrates previously run through the convection oven.

\subsubsection{Results and Discussion}

Despite improving the resistance slightly, the initial samples run through the convection oven still did not achieve similar resistance values to those of the box oven (Table 5). Furthermore, testing did not show a direct correlation between longer times in the oven and better performance. In fact, samples that had been run for at a speed of $15 \mathrm{~cm} / \mathrm{min}$ showed significantly poorer performance than samples that had been run twice at 30 $\mathrm{cm} / \mathrm{min}$. Theoretically these samples should be equal in resistance values as they had spent the same amount of time in the oven, however this was not the case. In order to test further the viability of the convection oven, additional samples were subjected to multiple runs through the oven. A matrix was developed to determine the optimal number of runs required to deem a sample fully cured (Table 6). A fully cured sample is defined as one that no longer reduces resistance by at least $15 \%$ upon re-curing (DuPont). 
Table 5. Resistance Improvements for Convection Oven Tuning.

\begin{tabular}{|l|c|c|c|c|}
\hline & \multicolumn{2}{|c|}{$100 \mu \mathrm{m}$ line } & \multicolumn{2}{c|}{$350 \mu \mathrm{m}$ line } \\
\hline & Avg & Stdev & Avg & Stdev \\
\hline Prev Box Oven 30min & 8.2 & 0.8 & 9.9 & 1.3 \\
\hline Box Oven 10min & 18.9 & 3.4 & 15.6 & 2.0 \\
\hline Convection $40 \mathrm{~cm} / \mathrm{min}$ & 28.4 & 6.4 & 18.1 & 6.4 \\
\hline Convection $30 \mathrm{~cm} / \mathrm{min}$ & 26.5 & 6.9 & 17.2 & 2.4 \\
\hline Convection $15 \mathrm{~cm} / \mathrm{min}$ & 22.0 & 2.9 & 16.3 & 2.0 \\
\hline Convection $30 \mathrm{~cm} / \mathrm{min}(\mathrm{x} 2)$ & 14.4 & 2.8 & 11.0 & 2.0 \\
\hline
\end{tabular}

Table 6. Matrix of Multiple Convection Oven Run Results.

\begin{tabular}{|c|c|c|c|c|c|c|}
\hline & \multicolumn{2}{|c|}{$\mathbf{1 0 0} \boldsymbol{\mu m}$ line } & \multicolumn{2}{c|}{$\mathbf{3 5 0} \boldsymbol{\mu m}$ line } & \multicolumn{2}{c|}{ \% decrease } \\
\hline \# of Runs & Avg & Stdev & Avg & Stdev & 100 & 350 \\
\hline $\mathbf{1}$ & 21.8 & 4.9 & 15.0 & 1.8 & - & - \\
\hline $\mathbf{2}$ & 12.2 & 2.8 & 9.8 & 1.2 & $-44 \%$ & $-35 \%$ \\
\hline $\mathbf{3}$ & 8.9 & 1.1 & 8.1 & 1.1 & $-27 \%$ & $-17 \%$ \\
\hline $\mathbf{4}$ & 7.2 & 0.7 & 6.8 & 1.0 & $-19 \%$ & $-16 \%$ \\
\hline
\end{tabular}

Upon running a total of 10 samples over a range of 1 to 4 runs, it was determined that 3 runs through the oven produced a fully cured sample with resistance values similar to those of the box oven (Figure 26). Although it is possible to still reduce resistance with additional runs, it is not considered a value add due to the insignificant decrease and the amount of time required.

It is suggested that the better performance from multiple runs, as opposed to longer time in the oven, is due to the overall air flow in the oven. By removing samples temporarily and reinserting them in the oven, the surface is exposed to an open environment and excess solvent is more easily removed. If not enough air flow occurs within a curing environment it is possible for the ink solvent to merely boil to the surface and not be carried away. This would result in the solvent resorbing into the ink upon cooling down 
again. However, because the Heller convection oven used is smaller than a typical industry grade convection oven (5 zones vs. 11 zones of heating) it is not possible to rule out the potential for a conveyor, convection oven to successfully cure a TPU printed sample with a single pass through.

For optimization of the Photonic Curing samples were sent to the machine manufacturer for assistance with tuning the parameters of the device. Upon testing of 28 total samples a final setting was arrived upon that produced results that both passed the tape test and produced the best resistance values up to this point in the experiment. These results showed that even after 15 minutes at $140 \mathrm{C}$ in a box oven, photonic curing could still be used to reduce resistance by up to $26 \%$. The final results from all three methods show that the combination of the box oven and photonic curing system achieved the best performance (Table 7). At this point all samples have been deemed to be fully cured as the calculated sheet resistance of the trace lines is less than the $75 \mathrm{~m} \Omega / \mathrm{square} / 25 \mu \mathrm{m}$ specified by the supplier.

Table 7. Final Curing Test Resistance Results.

\begin{tabular}{|l|c|c|}
\hline & \multicolumn{2}{|c|}{ Average Resistance Value } \\
\hline Curing Method & 100 um & 350 um \\
\hline Box Oven & 8.2 & 9.9 \\
\hline Convection Oven & 8.9 & 8.1 \\
\hline Photonic Curing & 9.4 & 8.6 \\
\hline PC after Box Oven & 6.7 & 7.2 \\
\hline
\end{tabular}

Results from the final reliability tests for the fully cured samples also show an improvement over the Phase 2 results (Table 8). For this crease test only samples that passed the tape test previously were used. Although the photonic cured samples do have a higher increase in resistance, the final resistance values for each method all fall relatively 
close to each other, thus showing that a sample must be fully cured for optimal

performance.

Table 8. Phase 3 Crease Test Results.

\begin{tabular}{|l|c|c|c|c|c|c|c|c|c|c|c|c|}
\hline & \multicolumn{3}{|c|}{100 um } & \multicolumn{3}{c|}{350 um } & \multicolumn{3}{c|}{ s100 um } & \multicolumn{3}{c|}{ s350 um } \\
\hline & before & after & \% increase & before & after & $\%$ increase & before & after & $\%$ increase & before & after & \% increase \\
\hline Box Oven & 16.7 & 43.7 & $162 \%$ & 16.2 & 25.9 & $60 \%$ & 16.0 & 21.6 & $31 \%$ & 20.0 & 26.1 & $35 \%$ \\
\hline Convection & 14.0 & 35.6 & $154 \%$ & 13.2 & 24.8 & $88 \%$ & 32.8 & 70.5 & $115 \%$ & 38.0 & 61.7 & $62 \%$ \\
\hline Photonic Curing & 10.1 & 37.2 & $268 \%$ & 11.0 & 27.1 & $146 \%$ & 22.7 & 52.7 & $132 \%$ & 25.7 & 49.3 & $91 \%$ \\
\hline
\end{tabular}

\subsubsection{SEM Imaging of Printed Inks}

In order to better understand the behavior of the ink and substrate interface samples were imaged in a Scanning Electron Microscope (Figure 29). The goal was to observe how densely packed the ink particles were and if voids could be seen. Furthermore, the interface and substrate were imaged to determine surface topography and spreading of the ink upon printing (Figure 30).
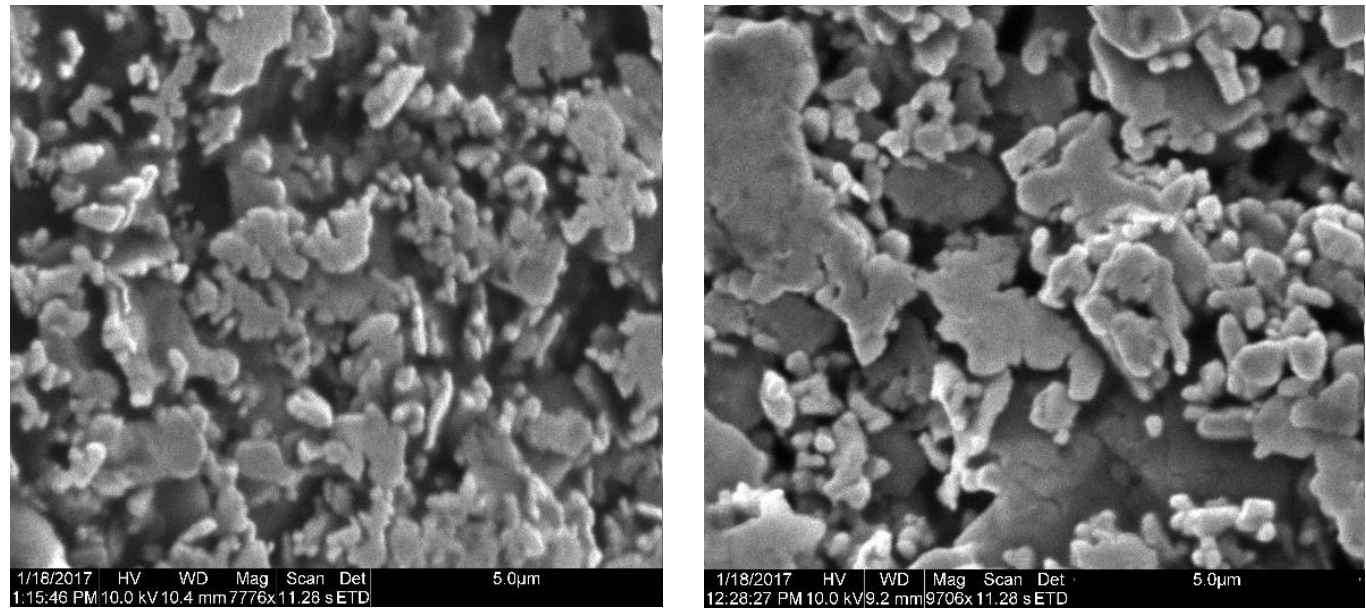

Figure 29. Images of the printed inks 5025 (left) and PE873 (right) show particle sizes ranging from 1-10 $\mu \mathrm{m}$ in size. The images are consistent with the information that the PE873 ink would have larger flake-like particles to allow stretch ability. 


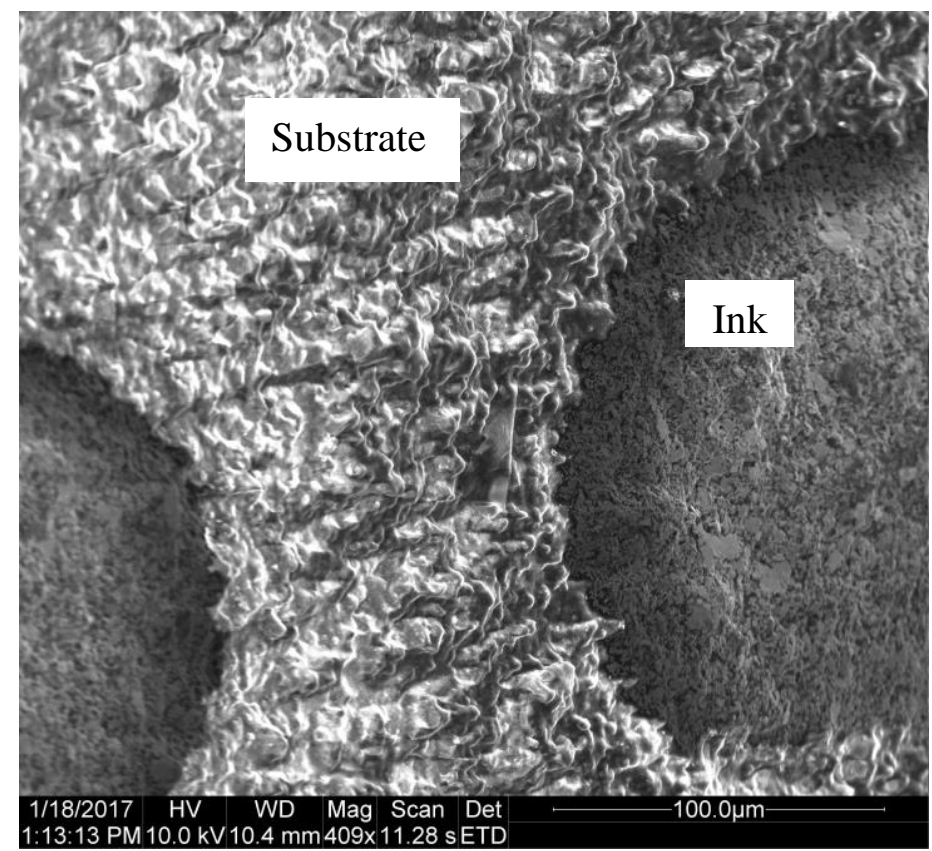

Figure 30. Interface of conductive ink and TPU substrate showing the large surface roughness of the substrate. Overall the ink appears to show little spreading after printing.

\subsection{Print Characterization Summary}

Based on the three curing methods available at Cal Poly, all curing methods have been optimized for achieving fully cured samples. It should be noted though that ultimately whichever curing method is used in industry will need to be tuned for the specific ink and substrate combination and if the samples are fully cured then resistance will not be affected. Of the three methods the photonic curing does offer the fastest time to fully cure, however this comes at the highest cost for machinery. Next steps for printing include further reliability tests across samples that have all been fully cured as it is unknown for certain whether the curing method has any effect on the stretch ability or performance of the samples. 


\section{CHAPTER 4. THINNED DIE CHARACTERIZATION REPORT}

\subsection{Objective}

The goal of the die characterization is to effectively determine the geometry and characteristics of each thinned die as received by the supplier including how thin each of the die is prior to bonding. The thickness of the die is directly related to how flexible it will be and thus how well it will perform under testing. It is important to evaluate the best method for characterizing the die without damaging them in any way. The thickness, warpage, and shape of the die will be critical to ensuring reliable manufacturing assembly and testing.

\subsection{Samples}

Two wafers, each consisting of 132 die measuring $10 \mathrm{~mm}$ square were ordered in order to perform the experiment. The first wafer was thinned to a target of $75 \mu \mathrm{m}$ thick, while the second was thinned to a target of $50 \mu \mathrm{m}$. The die were delivered to the team in both standard waffle packs, as well as specialized tacky waffle packs provided by GelPak. Each die consists of a fixed amount of leads which make up a daisy chain circuit. The core of the die has 16 mil pitch in both directions (Figure 31). The bumps on the die are plated with a NiAu plating and have a square shape, with a flat surface finish (Figure 31). The primary die used for thickness characterization purposes came from the second wafer of target $50 \mu \mathrm{m}$. 


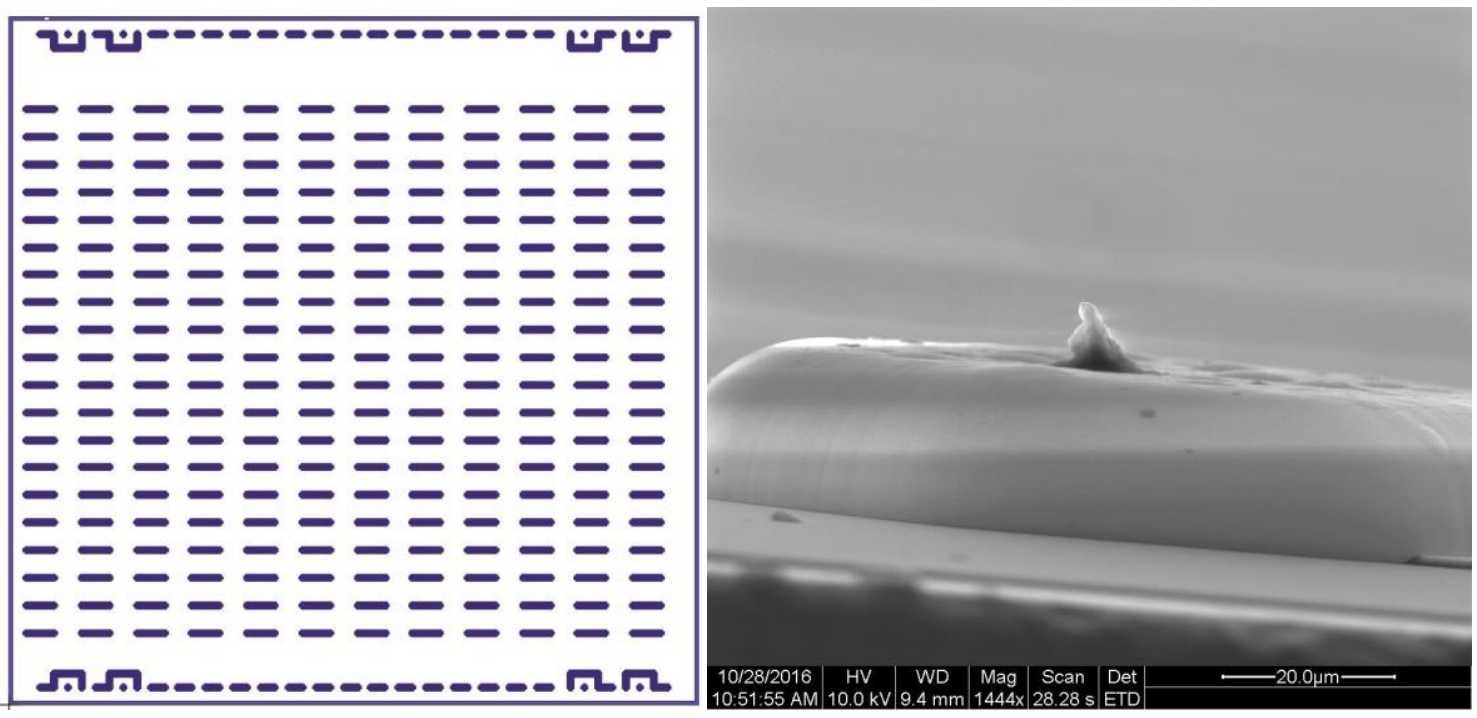

Figure 31. Top view of PacTech 2.3 die with $10 \mathrm{~mm}$ side length (left). SEM image of flat NiAu bump on PacTech (right).

\subsection{Characterization Methods}

There are two primary categories of characterizing the die: physical displacement measurement and microscopy techniques. Both are nondestructive for the most part, however the more physical measurements taking place the greater likelihood of a potential for failure to occur. Additional destructive methods for characterizing die strength and properties exist, however at this point those methods are not being consider given the small supply of available die. The methods chosen for characterization include stylus profilometry (physical) and microscopy (SEM and optical). Both are described in more detail below

\section{Stylus Profilometry}

The simplest method for determining geometry of the die is using profilometry and physical deflection. Stylus profilometry is a well-known procedure that analyzes topographical displacement values by dragging a small tip across the surface of a sample. 
Previous tests using profilometry on the thinned die samples have given results that contradicted the expected values for thickness, therefore it is essential to understand the nature of how the profilometer generates data when using it for characterization. The profilometer has several variables that can be adjusted to calculate displacement values including feature shape, feature size range, drag force, scan speed, and scan length. Each one affects the final data readout and contributes to the resolution of the $\mathrm{z}$ axis measurements.

In order to begin characterizing the overall shape of the die, stylus profilometry was used, measuring the step down from the die edge (Figure 32). The measurement values resulted in an average die thickness of $\sim 85 \mathrm{um}$, and therefore additional characterization was attempted in order to verify or disprove these results.

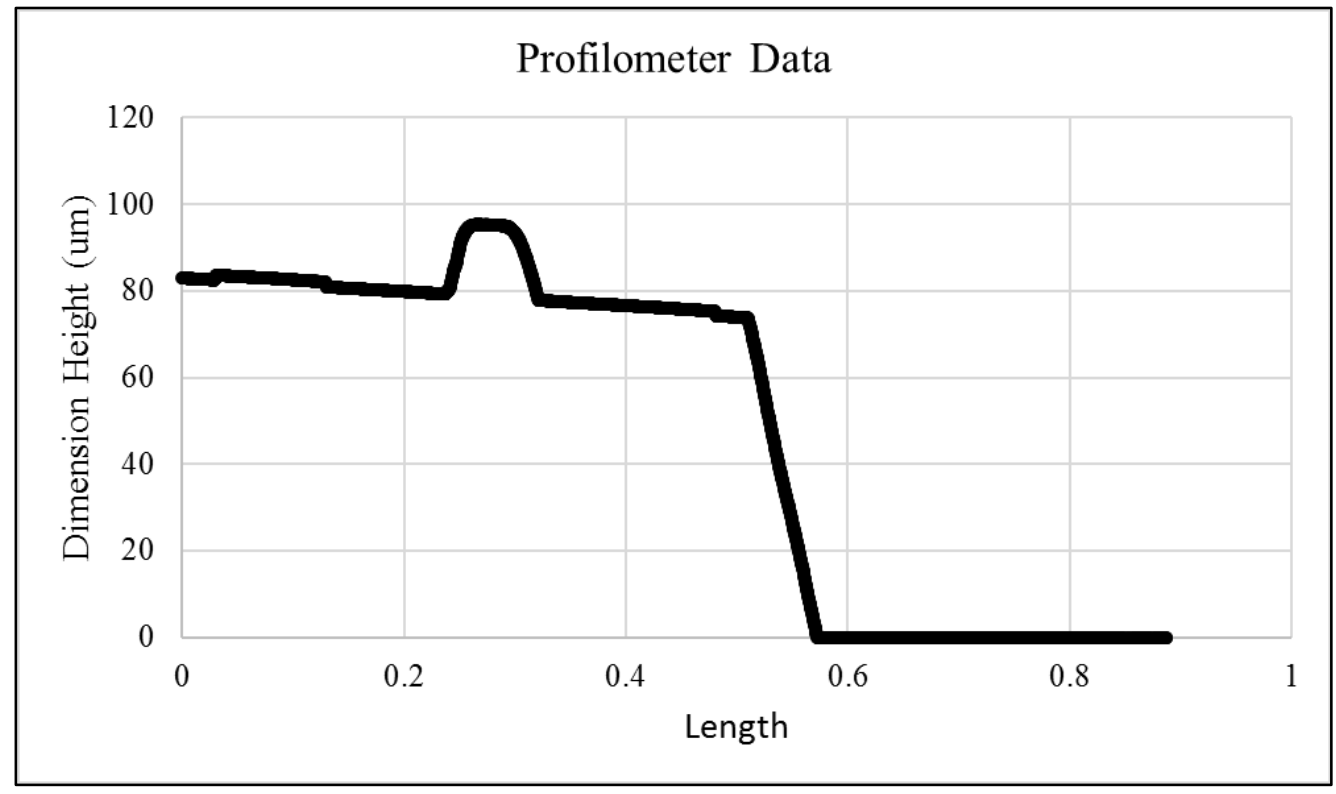

Figure 32. Profilometry performed on the thinned die revealed a die thickness of roughly 80 $\mu \mathrm{m}$. The bump shown is one of the edge bumps on the die.

Further characterization involved imaging the die via SEM. The die was mounted vertically in order to view a cross section of the thickness. These results produced an estimated thickness of around $65 \mu \mathrm{m}$, which is slightly larger than the target thickness of 
$50 \mu \mathrm{m}$, but assumes the die was imaged perfectly perpendicular which is not necessarily the case (Figure 33).

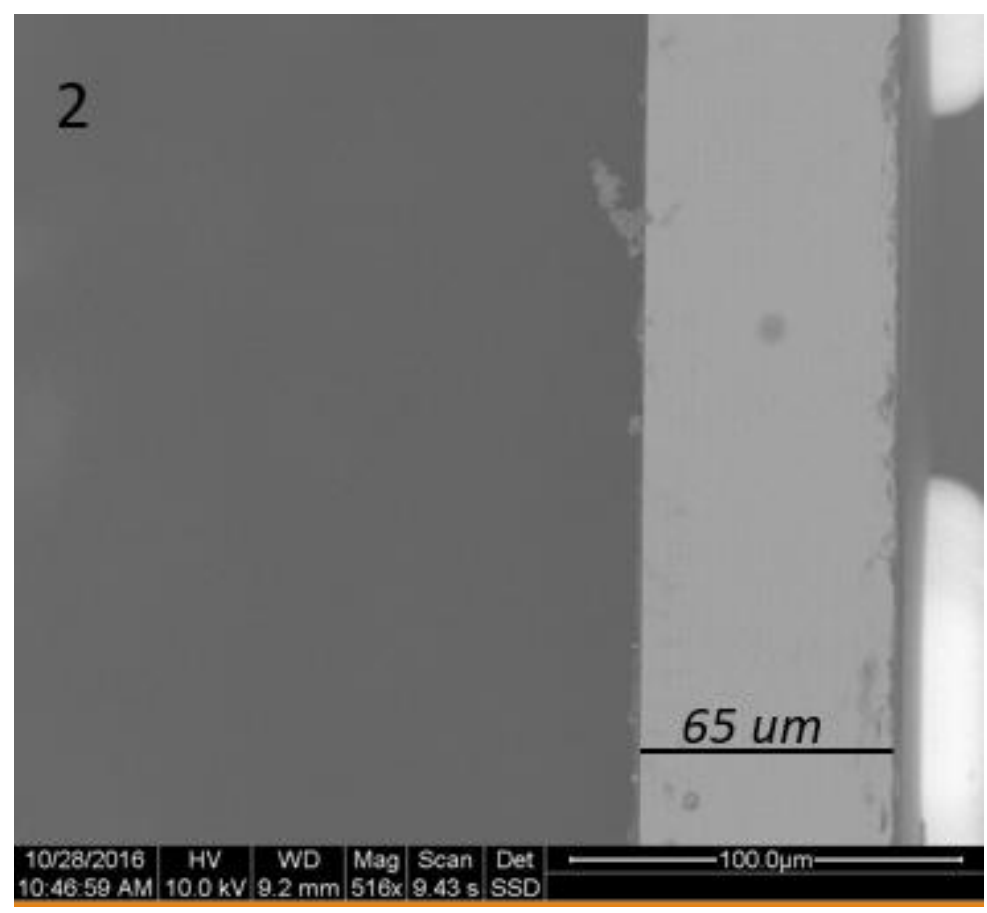

Figure 33. Cross section of thinned die in SEM with thickness measurement for reference.

Because this characterization using profilometry showed die thickness to be close to 80 $\mu \mathrm{m}$, whereas SEM characterization verified the thickness at $65 \mu \mathrm{m}$ a more complete measurement system was needed. Both machines were correctly calibrated therefore the error in profilometry is likely due to warpage of the die causing liftoff of the measured edge (Figure 32, Figure 33).

As for measuring the die, a distinct measurement plan was fabricated to gather information for both die thickness and die warpage. Previous tests had shown that any warpage in the die could lead to false readings in thickness values, as the edge of the die used for the step-down measurement might not be fully flush with the stage. Therefore, if a profilometer reading were taken the resulting value would be much greater than the actual thickness of the die. To achieve the best possible measurement, it was decided to 
measure thickness at the corners of the die where the least observable warpage occurred

(Figure 34). It is important to note the assumption that thickness uniformity across the die is negligible.

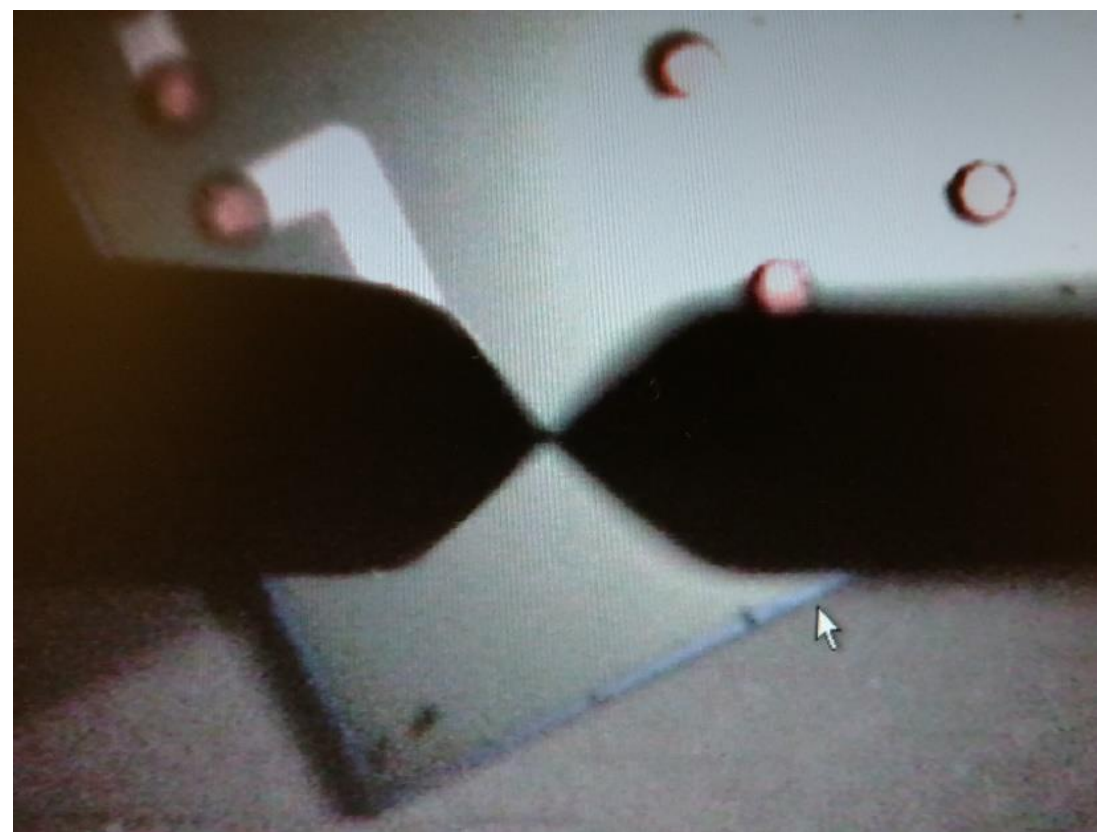

Figure 34. Camera image of stylus profilometer measuring the corner of the die which is assumed to have the least amount of warpage across the surface. Prior to beginning the scan off the die the stylus is lowered until contact is made with the die. Occasionally during this lowering the die corner was observed to be pressed down until contact with the stage was made.

For measuring overall warpage, the goal was to achieve a three-dimensional model of the die, however this is not ideal for a manufacturing facility that wants to quickly ensure the flatness of a given die. Therefore, the chosen method was to measure a single pass vertically and horizontally through the center of the die (Figure 35). By following these lines, the readout data would give a general idea about the shape of the die with respect to a flat stage. Ideally one would perform multiple passes in each direction if an overall shape of the die were sought. 


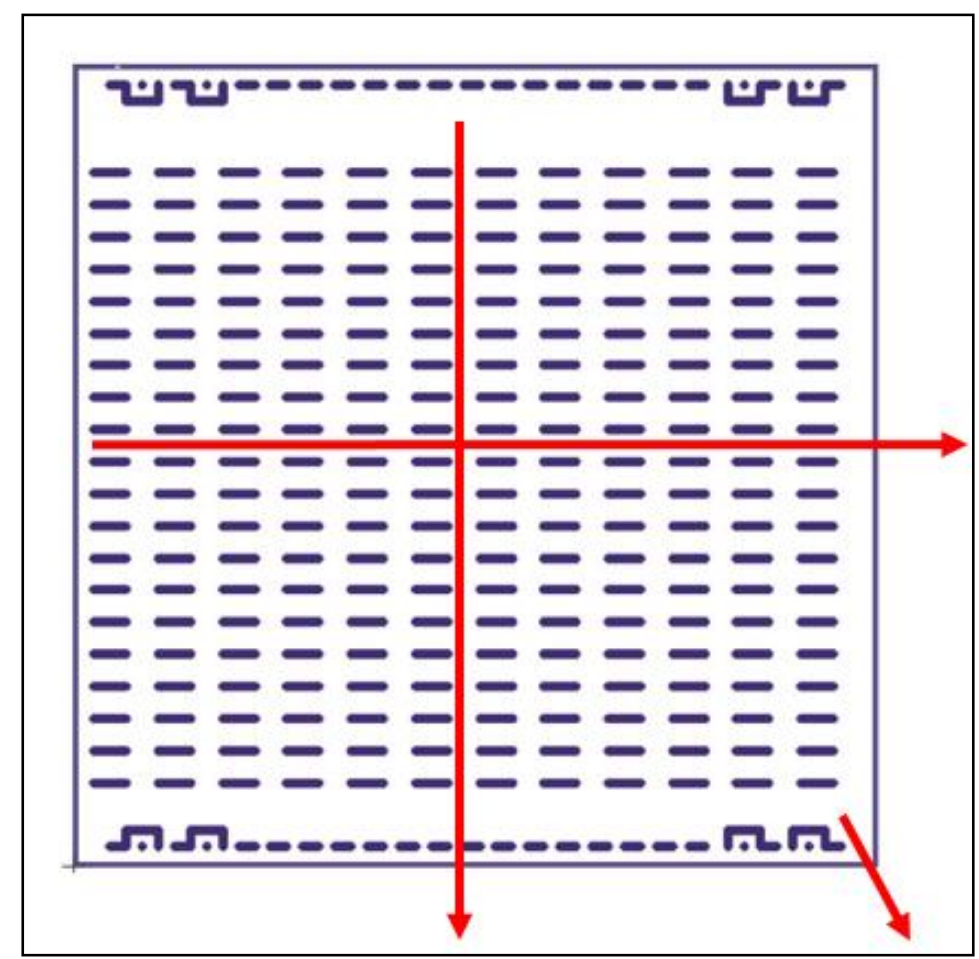

Figure 35. Profilometer map depicting the 3 measurement paths taken by the stylus to measure die thickness and overall warpage. The challenge for this measurement is avoiding tracing over any bumps which might alter the thickness data.

One additional challenge with using stylus profilometry is adhering the die to the stage.

Due to the likelihood of the die moving while the stylus is being dragged across, the die must be fixed in some manner. However, there cannot be any exterior stresses applied (i.e. tapped down) or else the warpage of the die would be affected. Therefore, the best method found was to place pieces of double sided tape flat on a microscope slide and push the die against the side of the tape (Figure 36). This method fixed the die without adding any stresses or leaving any residue on the die for later assembly. Multiple die were adhered simultaneously to assist in profilometer throughput. 


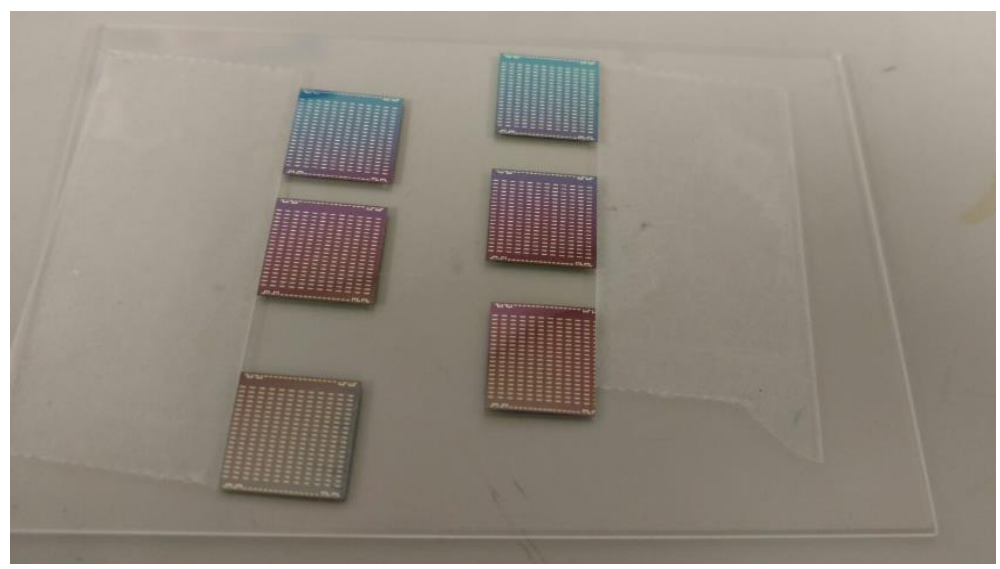

Figure 36. Die tapped against the edges of double sided tape allow for ease of measuring without affecting the internal stresses of the die.

\section{Microscopy}

The methods for microscopy being used can be divided into 3 subcategories: traditional bright-field microscopy, scanning electron microscopy (SEM), and advanced machine based optical characterization. The primary sources used at Cal Poly that pertain to this thesis are optical and SEM methods. For traditional optical microscopy, the purpose of use was to verify large scale dimensions and surface defects of the die. SEM on the other had was used to verify more fine dimension features as well as observe the microdefects present in the die.

Upon discussion with the industry partners it was suggested to use a laser system to methodically and quickly measure the displacement over the die surface. Initially a MicroVu system was suggested, of which Cal Poly does have on hand. However after testing at the partner facility the MicroVu system was deemed unreliable in favor of an OGP system. Being that Cal Poly does not own an OGP system, the majority of this type of characterization will be conducted at the industry partner's facility. 


\subsection{Die Warpage}

One key aspect to take into account is die warpage. Any potential warpage will greatly affect the die attachment process once final assembly begins. The team attempted to use a confocal microscope in order to image the die in space, however by needing to tape down the die for adhesion, any warpage was counteracted. Furthermore profilometry was conducted over an entire sample, giving a profile of the die and a good idea for exactly how much warpage was taking place (Figure 37). It is important to note that even with the edges appearing to be over $100 \mu \mathrm{m}$ tall, this does not take into account the warpage in the opposite direction which would raise that edge (Figure 38).

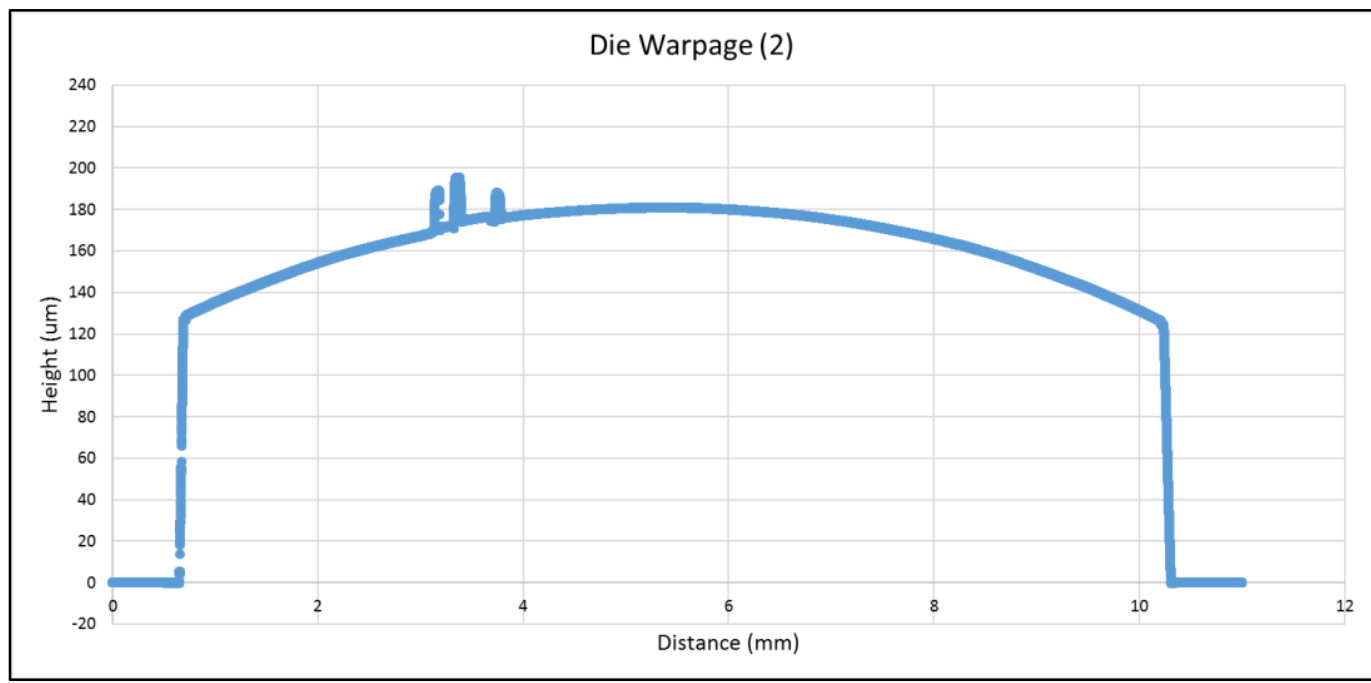

Figure 37. Profilometry profile of entire die showing amount of warpage in one linear direction. 


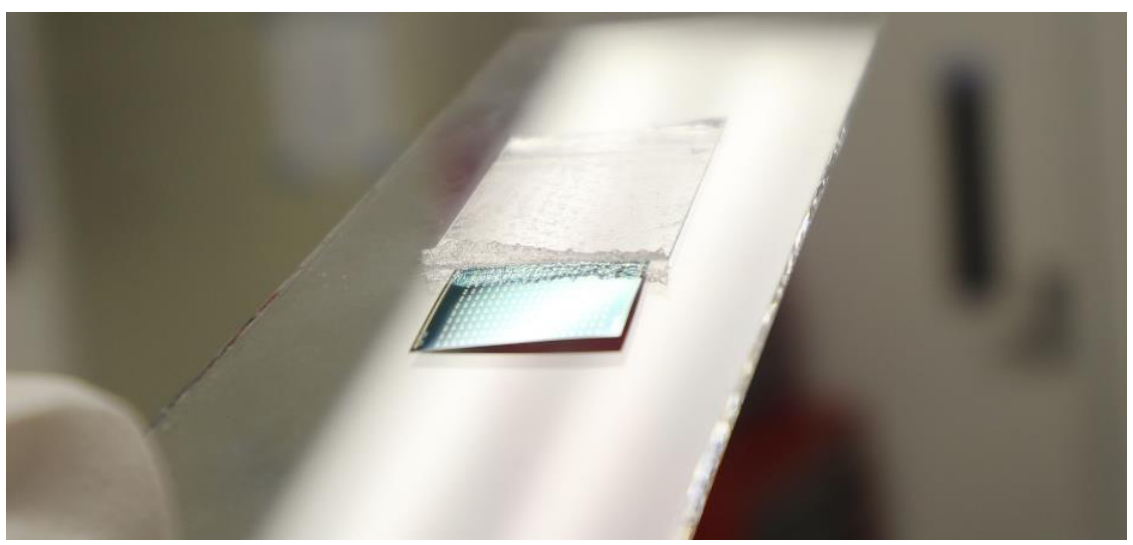

Figure 38. Due to the internal stress in the silicon die, warpage can be viewed by visual inspection alone.

\subsection{Results and Discussion}

The profilometry of the thickness for the die all showed a distinct, yet repeated pattern in their overall shape (Figure 39). The thickness of the die was measured to be the exact point at which the curvature of the die fell off. Based on these measurements for a sample of 21 die statistical values were calculated (Figure 40).

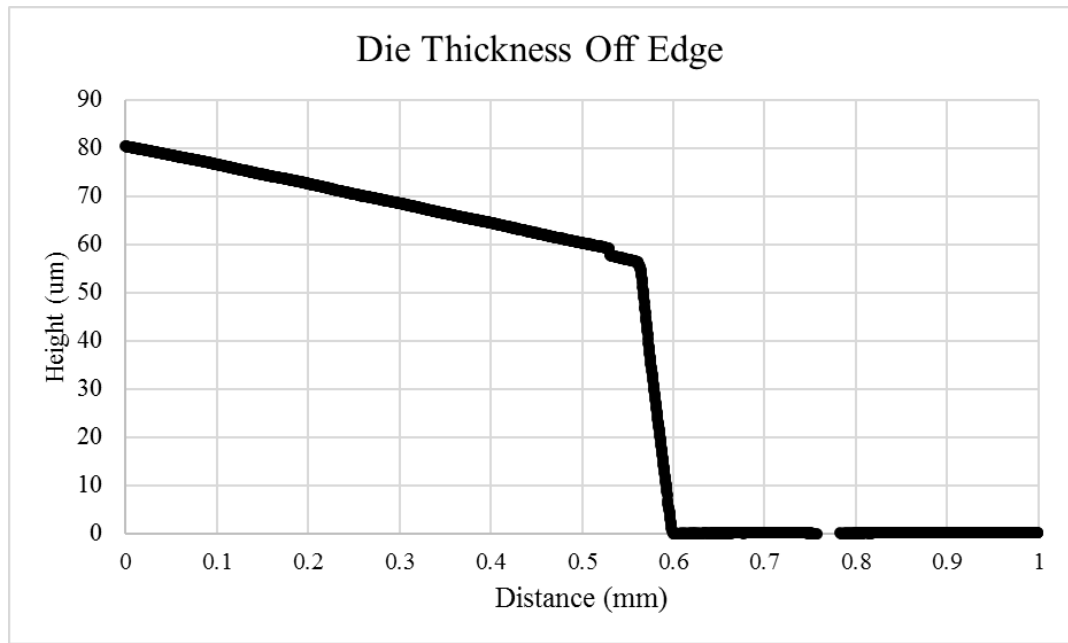

Figure 39. Die thickness side profile showing sloping of die warpage and edge thickness of die. 


\section{Descriptive Statistics: Thickness \\ Variable N $N^{*}$ Mean SE Mean StDev Minimum Q1 Median Q3 Maximum \\ $\begin{array}{llllllllll}\text { Thickness } 21 & 0 & 39.12 & 1.59 & 7.31 & 28.64 & 32.84 & 37.68 & 46.12 & 51.04\end{array}$}

Figure 40. Descriptive statistics of die thickness. Given a target thickness of $50 \mu \mathrm{m}$ the process does not show consistency in uniformity or mean.

The results from the thickness measurements were of concern given that the target thickness of $50 \mu \mathrm{m}$ produced a sample mean of $39 \mu \mathrm{m}$, and that the standard deviation was so large. The large deviation, which has a potential effect on warpage, can cause issues during assembly as varying thicknesses of die can withstand varying levels of stress. There could be potential for large amounts of die failure due to cracking if the process were calibrated on a thicker die that had been able to withstand greater forces. In order to verify the sample thickness values a die measuring $29 \mu \mathrm{m}$ on the profilometer was imaging using the SEM (Figure 41). The results showed a die that indeed had a thickness in the $30 \mu \mathrm{m}$ range. Interestingly however the die showed distinct thickness difference along the side, ranging from $28 \mu \mathrm{m}$ on one end to $31 \mu \mathrm{m}$ on the other. This difference could partially be due to positioning in the SEM holder, however an actual $10 \%$ in die uniformity would be of great concern given the unpredictability of die behavior it could lead to including warpage, fracture, and poor reliability performance. 


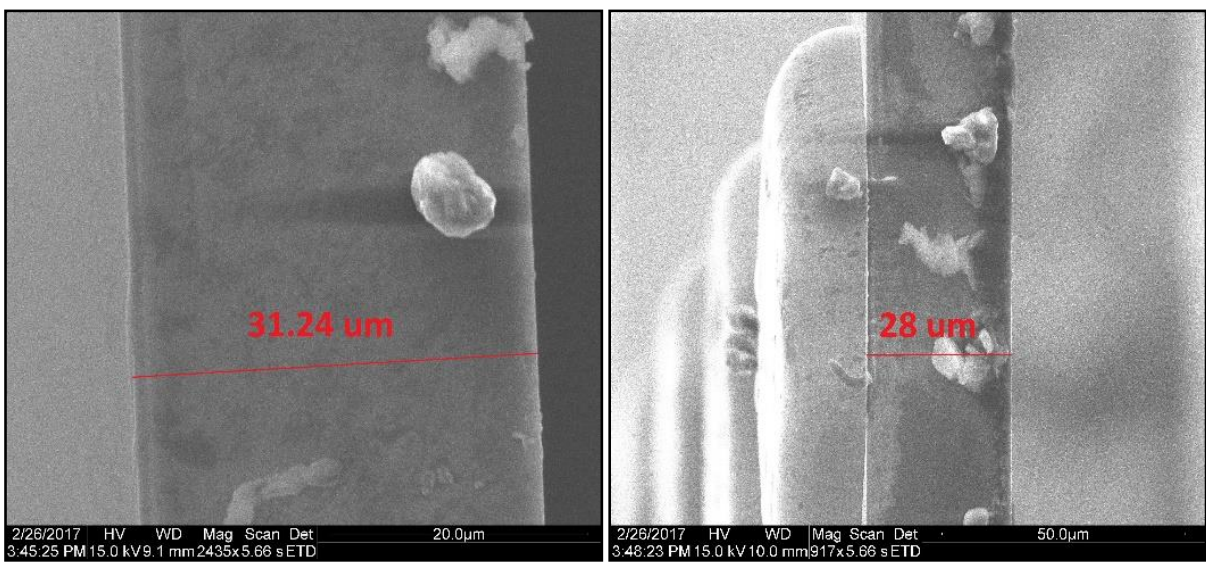

Figure 41. SEM image of a thinned silicon die showing the side thickness and its variation across the $10 \mathrm{~mm}$ side of roughly $10 \%$.

After conducting a series of profilometry measurements, a generic pattern began to show in the die thickness and warpage results. Initially the die were measured using an $11 \mathrm{~mm}$ trace distance in order to capture both sides of the die (Figure 42). However, it became difficult to successfully measure each die without dislodging it from the tape, or recording erroneous results. In order to better filter out the warpage data from the thickness data a new method was determined.

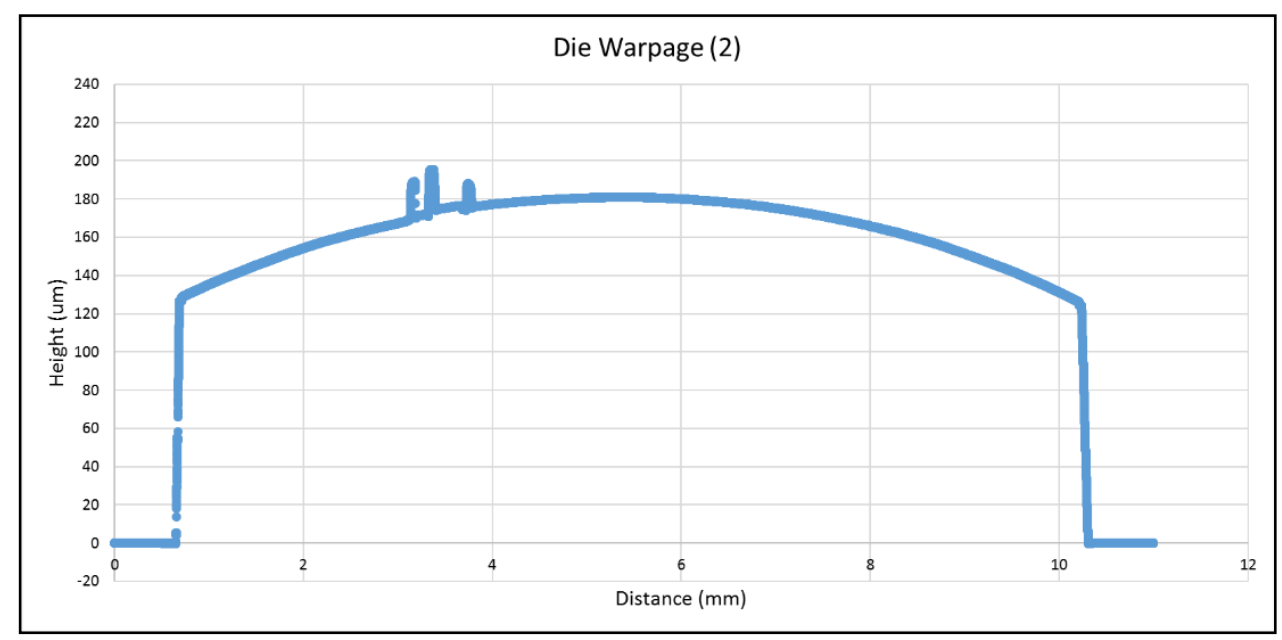

Figure 42. Original profilometry profile of entire die showing amount of warpage in one direction. $\mathrm{Z}$ axis results include both warpage and die thickness making it difficult to determine the exact values for each.

The new method for measuring warpage began by starting the profilometry trace at the edge of the surface of the die then dragging over the entirety and off the other side. The 
original starting point would then be considered the 0 position and would be leveled with the other edge of the die (Figure 43). The warpage would then be recorded as the maximum height between the two zero points.

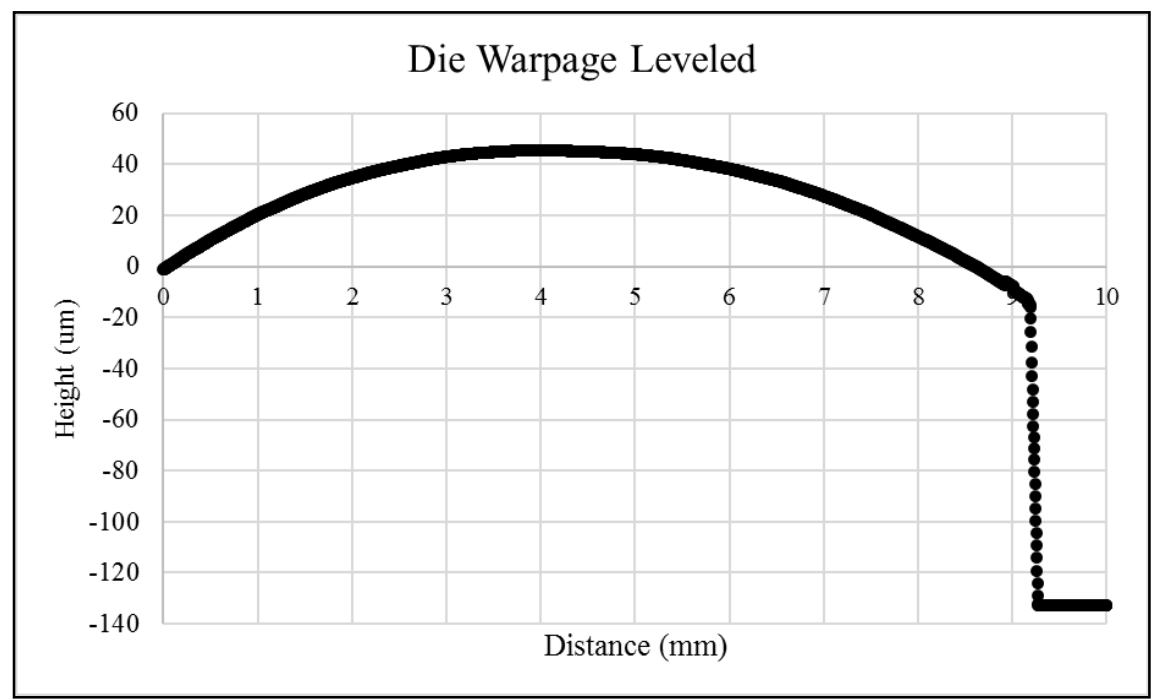

Figure 43. By leveling the edges of the die measurements at zero the overall warpage can be characterized as the height above the $\mathrm{x}$ axis.

When looking at the warpage results it became evident that certain die had significantly more warpage than others. To better understand the behavior of the die the warpage was plotted with respect to thickness (Figure 44). The data showed a somewhat linear trend with thinner die containing larger amounts of warpage than thick die. This trend suggests that the die have inherent internal stresses and as more silicon is removed those stresses begin to have a greater visible effect. 


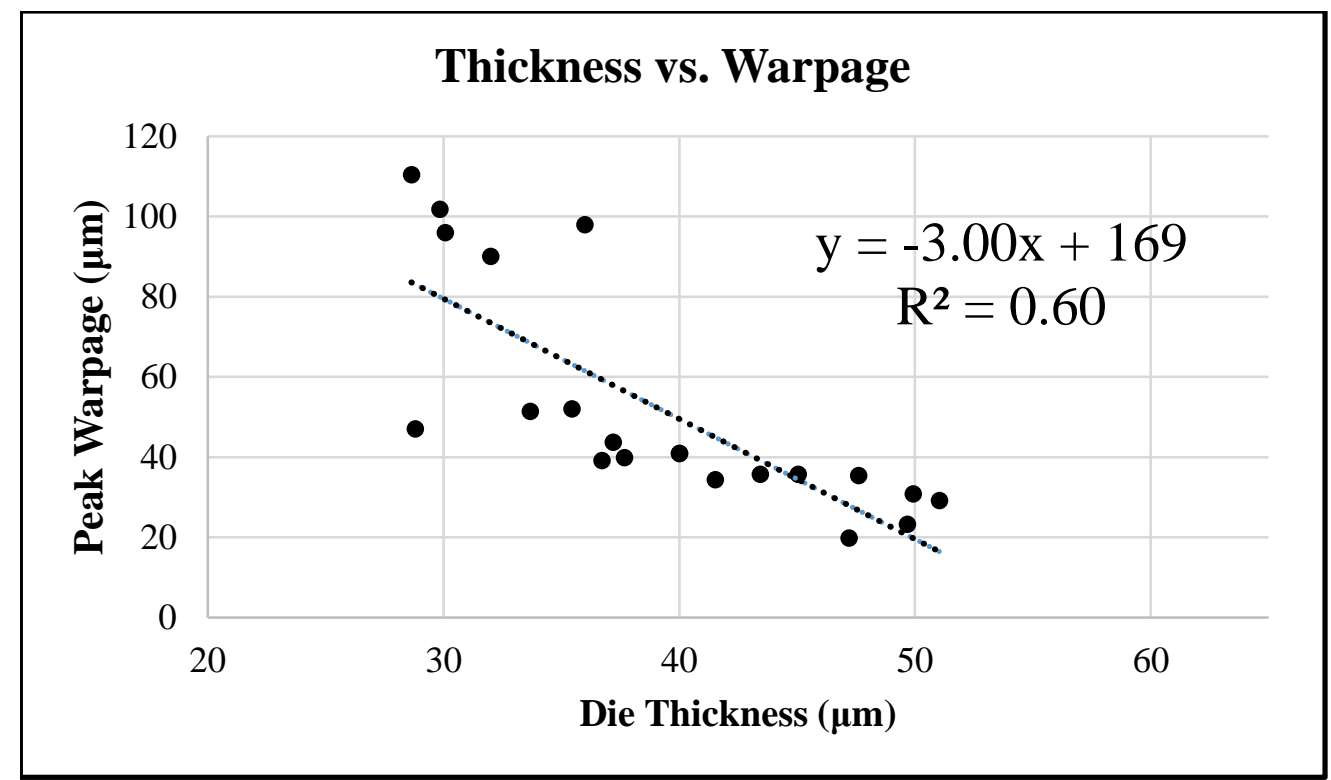

Figure 44. Trend showing die thickness vs. die warpage. Roughly $20 \%$ of the die received contained significant amounts of warpage with thinner than expected thicknesses.

By looking at the data it is clear to see that roughly $20-25 \%$ of the die are proportionatly thinner and more warped than others. This non-uniformity is again concerning for the yield numbers of the manufacturing process once assembly begins. As the project continues it will be essential to work with the supplier or determine a new supplier for achieving better uniformity in the die thicknesses.

It is important to note also that using the advanced laser optical measurment tool at the industry partner facility that the ultra thin die had even larger warpage readings, upwards of $170 \mu \mathrm{m}$. This discrepency in the data is likely due to the limitiations of the stylus profilometer. For one, the profilometer is making contact with the surface and therefore could be likely compressing the die that have large amounts of flexiblity. Also when measuring the warpage the profilometer was set to a target range of $100 \mu \mathrm{m}$ which has a maximum readout value of $120 \mu \mathrm{m}$, therefore it is possible some values towards the upper extreme limit of $120 \mu \mathrm{m}$ could have been incorrectly adjusted in the machine. 


\subsection{Conclusion}

Overall the die characterization has provided a general understanding of the geometry and behavior of the die without performing extensive destructive testing. The greatest concern is the large amounts of warpage and the invariability seen in the thickness and warpage recordings. Additional suppliers will need to be investigated in hopes of achieving less warpage and better thickness uniformity across the die. The linear relationship between thickness and die warpage is likely due to increased levels of internal stress. Reducing this stress as much as possible is necessary to achieve die warpages acceptable for assembly. 


\section{CHAPTER 5. ADDITONAL WORK, SUMMARY, AND CONCLUSIONS}

\subsection{Other Work}

\subsubsection{Copper Clad Samples}

One additional point of work that has yet to be a focus of this project was the characterization of samples of flexible copper on TPU. These samples were ordered in order to test the ability to solder (either via a reflow oven or with photnic solder) thin die to a flexible substrate. The samples were ordered from supplier with a NiAu coating and used the same pattern features as the printed samples in order to allow for comparison using reliability tests (Figure 45).

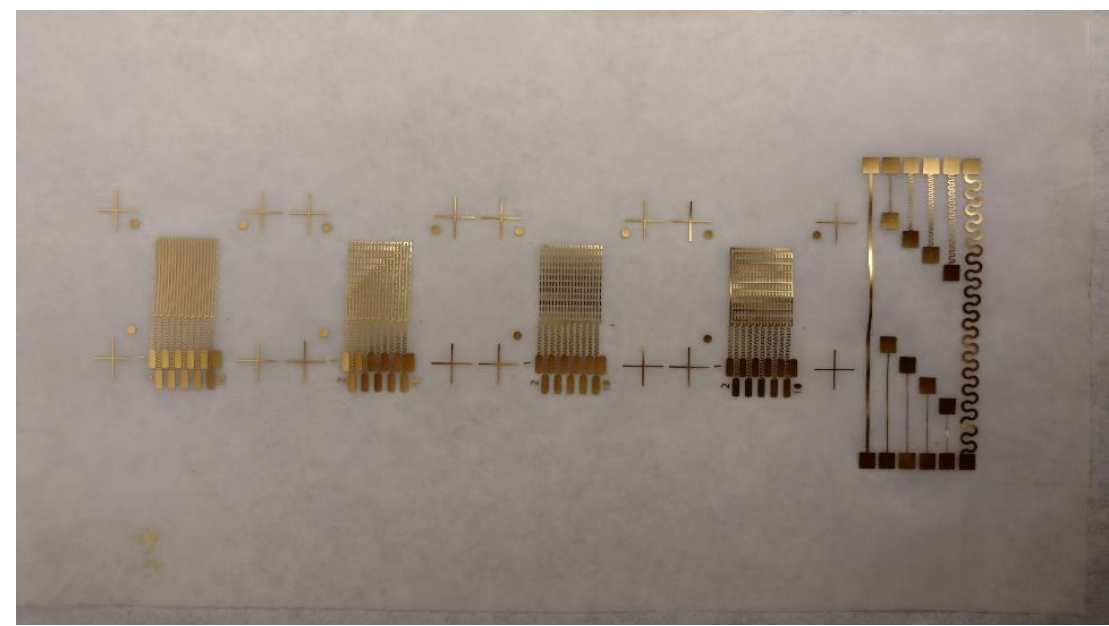

Figure 45. Copper clad samples on TPU as prepared by supplier. The copper shows noticeable restrictions on the substrate when small forces are applied.

Characterizing the $\mathrm{Cu}$ clad samples shows them to have an average height of $30 \mu \mathrm{m}$ with a TPU thickness of 2 mils (Figure 46). The Cu supplied TPU samples are different than the Bemis TPU as the surface roughness is closer to 3-4 $\mu \mathrm{m}$ (vs. 6-8 $\mu \mathrm{m}$ for Bemis), and the TPU is slightly transparent as opposed to the opaque white of the Bemis TPU. When imaging under SEM the samples show excellent feature definition, with minor roughness 
at the TPU interface (Figure 46). Future tests will need to be conducted to determine the adhesion capabilities of the $\mathrm{Cu}$ to the TPU.
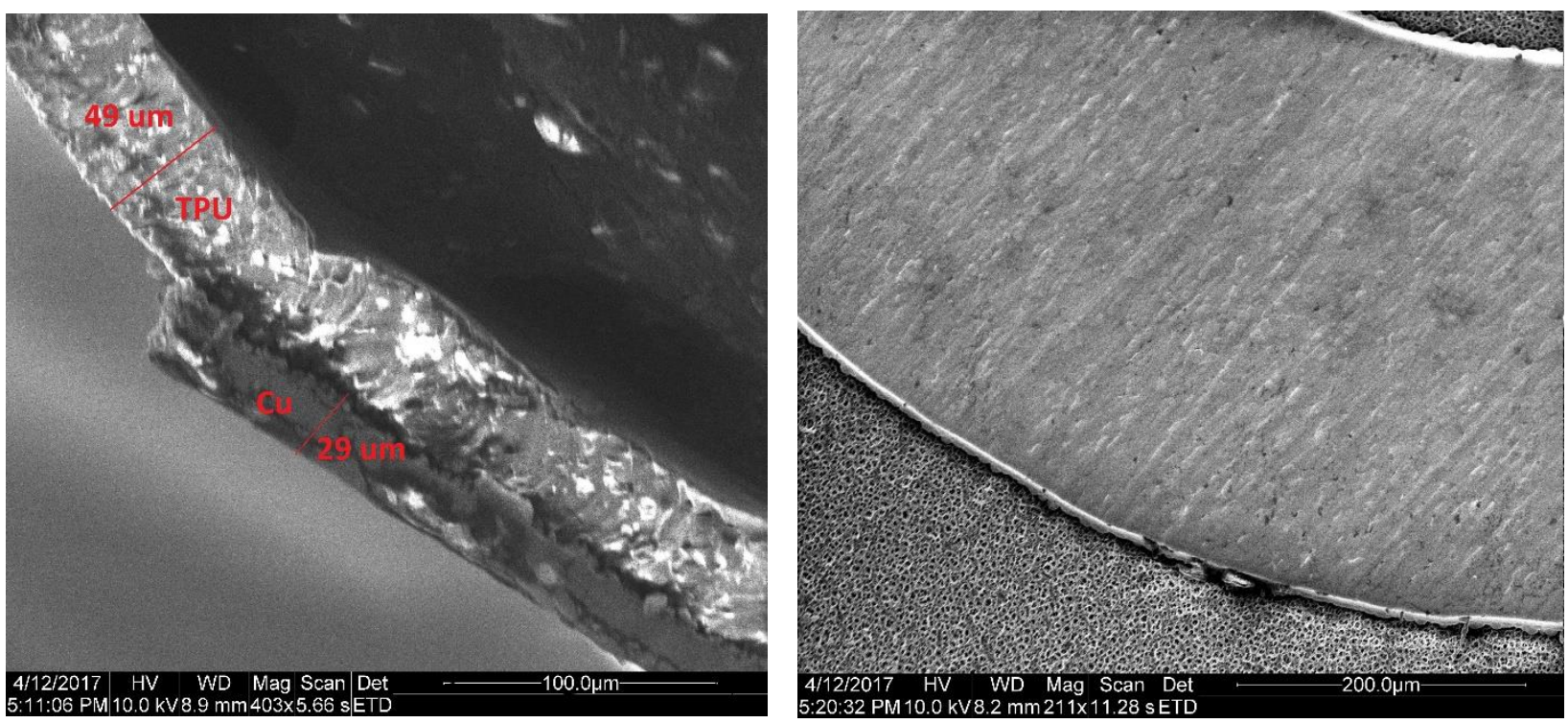

Figure 46. SEM images of Cu clad samples on TPU. Copper thickness and TPU thickness measured support expected thicknesses (left). The rough interface and porous TPU surface may pose a challenge for future assembly tests (right).

\subsubsection{Assembled Devices}

Extensive work has been completed on the development of the attachment and assembly of the thin die to the flexible substrate, however much of this work has been performed at the Jabil Inc. facility in San Jose and is outside the scope of this thesis project. However with regards to the characterization of this process several key discoveries have been made. Gathering information took place via trips to the Jabil facility and preliminary attachment of the die using ACA and ACF look promising for assembly. One big challenge in terms of manufacturing scalability that has been discovered is with regards to removing the supporting plastic liner from the substrate post die attachment. With the current manufacturing system the substrate is batch processed via three separate machines for the die attachement procedure. Only after successful bonding of the die is the liner able to be removed. However due to the fragility of the die and substrate and the 
difficulty of removing the back liner this task remains difficult and little investigation has been taken into account on how to best remove the liner. Ideally in a large scale manufacturing process the die attachment process could be converted into a roll-to-roll manufacturing system that would allow for the removal of the liner in-situ without needing to take additional steps. However for now the liner must be indiviudally peeled back using extreme caution in order to preserve the attached die. Likewise no tests have been performed to see how easily these assembled devices behave when adherred to fabric.

\subsection{Future Work}

The next main steps for characterization include utilizing the Instron testing fixture that has been manufactured and delivered by the mechanical engineering team to characterize the perfomance of the ink under a variety of stressing scenarios and configurations. The results will need to be compared to the manufacturers specifications that claim up to 30$40 \%$ strain with only $100 \%$ resistance increase. Furthermore the additional die that have been ordered are being thinned using a different supplier. These die thicknesses and variations will need to be characterized prior to assembling of devices. This supplier specifies a manufacturing tolerance of $+/-5 \mu \mathrm{m}$ for a $50 \mu \mathrm{m}$ target thickness. Likewise a model needs to be developed to determine how much warpage is allowed in a thinned die, in relationship to the overall thickness, in order to not affect yield during manufacturing. Preliminary assembly showed favorability towards cracking with die that exhibited higher warpage. 
Once the assembly process has been finalized and the devices are being manufactured in larger quanitites, a thorough and complete characterization and reliability test will need to be conducted. A sample size of at least 300 assembled devices is needed to thoroughly characterize the behavior and establish worthy reliability metrics for the overall project. These tests will include not only strain tests but also humidity tests, thermal cycling tests, and resistance under strain tests. Once the devices are fully characterized, a better idea of product yield and feasibility can be determined. This theoretical yeild will serve as the guidline for reporting the manufacturing capabilities to industry partners. Given that little to no testing has been conducted on thinned die as large as $10 \mathrm{~mm}$, the opportunities for development are plentiful, as larger die open up the possiblity for more complex ICs.

\subsection{Summary and Conclusion}

The characterization of the printed traces and thinned die have led to many developments and discoveries about FHE capabilities. By conducting through analysis and careful procdure during the printing process, the optimal printing parameters of lower speed, lower pressure, and thicker emulsion were concluded. Likewise, by conducting a comprehensive review of the three separate curing methods, an optimized output for curing settings was delivered for each method. Stretchable ink samples printed on TPU can now be cured via batch process in a box oven, continuously in a convection oven, or rapidly in a photonic curing system. All three methods achieve the manufacturer's sheet resistance specifications and perform equally under reliability tests. This printing process is now ready to be adopted by a large manufacturer should they have the desire to do so. 
For the thinned silicon die, results have been collected that show documented behavior of die with large aspect ratios that have not been analyzed prior. The linear trend of thinner die having higher warpage shows just how detrimental internal stress can be at thicknesses below $100 \mu \mathrm{m}$. Furthermore, future data collected will be able to verify the necessary thickness uniformity requirements to avoid die fracture during and after assembly. Future work conducted on both the conductive adhesive attachment methods and the $\mathrm{Cu}$ clad assembly will provide vital information to individuals seeking to design devices in the realm of FHEs. 


\section{REFERENCES}

[1] S. Brand, "Flexible Hybrid Electronics (FHE) is the Next Big Thing in Manufacturing: An Entirely New Platform for Electronics," CMTC Creative Solutions, 16 Nov 2016.

[2] D. Kim and M. Jooho, "Highly Conductive Ink Jet Printed Films of Nanosilver Particles for Printable Electronics," Electrochemical and Solid-State Letters, no. 8, pp. J30-J33, 2005.

[3] "Flexo Printing has a future in printed electronics," Flexo Gravure Global, 25 January 2016. [Online].

[4] D. O. R. Tobjork, "Paper Electronics," Advanced Materials, 2011.

[5] T. Morrison, J. Silver and B. Otis, "A Single-chip Encrypted Wirelss 12-Lead ECG Smart Shirt for Continuous Health Monitoring," in IEEE Symposium on VLSI Circuits Digest of Technical Papers, 2014.

[6] G. Salvatore, N. Munzenrieder, T. Kinkeldei, L. Petti, C. Zysset, I. Strebel, L. Buthe and G. Troster, "Wafer-scale design of lightweight and transparent electronics that wraps around hairs," Nature Communications, 2014.

[7] S. B. Fuller, E. J. Wilhelm and J. M. Jacobson, "Ink-Jet Printed Nanoparticle Microelectromechanical Systems".

[8] M. A. Yokus, R. Foote and J. S. Jur, "Printed Stretchable Interconnects for Smart Garments: Design, Fabrication, and Characterization," IEEE Sensors Journal, vol. 16, no. 22, pp. 7967-7967, 2016.

[9] M. Gonzalez, F. Axisa, M. V. Bulcke and J. Vanfleteren, "Design of metal interconnects for stretchable electronic circuits," Microelectronics Reliability, vol. 48, no. 6, pp. 825-832, 2008.

[10] H. Merchant, J. Wang, L. Giannuzzi and L. Y.L., "Metallurgy and performance of electrodeposited copper for flexible circuits," in EUROTOM '99, Munich, 1999.

[11] A. Shchepetov, M. Prunnila, F. Alzina, L. Schneider and J. Cuffe, "Ultra-Thin FreeStanding Single Crystalline Silicon Membranes With Strain Control," Applied Physics Letters, vol. 102, no. 19, 2013.

[12] J. P. Gambino and I. Microelectronics, "Thin Silicon Wafer Processing and Strength Characterization," in 20th IEEE International Symposium on the Physical and Failure Analysis of Integrated Circuits, 2013. 
[13] C. Landesberger, N. Palavesam, W. Hell, A. Drost, R. Faul, H. Gieser, B. Bonfert, K. Bock. "Novel processing scheme for embedding and interconnection of ultrathin IC devices in flexible chip foil packages and recurrent bending reliability analysis," in Electronics Packaging (ICEP), Sapporo, Japan, 2016.

[14] SPT, "Chip Bonding Tools Tools," SPT-Group small precision tools, 2017. [Online].

[15] R. van Silfhout, W. van Driel, Y. Li, G. Zhang and L. Ernst, "Prediction of BackEnd Process-Induced Wafer Warpage and Experimental Verification," in Electronic Components and Technology Conference, San Diego, 2002.

[16] N. Sudani, K. Venkatakrishnan and B. Tan, "Laser singulation of thin wafer: Die strength and surface roughness analysis of $80 \mathrm{um}$ silicon dice," Optics and Lasers in Engineering, vol. 47, pp. 850-854, 2009.

[17] S. Seok and J. Kim, "A study on effect of wafer bow in wafer-level BCB cap transfer packaging," Microsystem Technologies , vol. 20, no. 2, pp. 215-219, 20134.

[18] D.-S. Lui, Z.-h. Chen and C.-Y. Lee, "Evaluate Breaking Strength of Thin Silicon Die by Ball-on-ring Microforce Tests and Finite Element Analysis," in International Microsystems, Packaging, Assembly and Circuits Technology Conference (IMPACT), Taipei, 2011.

[19] Panacol.com, "Adhesives with Electrical Conductivity for Applications on PCBs," Panacol-Elosol GmbH, 2017. [Online].

[20] J. Niittynen, V. Pekkanen and M. Mantysalo, "Characterization of ICA Attachment of SMD on Inkjet-Printed Substrates," in Electronic Components and Technology Conference (ECTC), Las Vegas, 2010.

[21] J. Niittynen, J. Kiilunen, J. Putaala, V. Pekkanen, M. Mantysalo, H. Jantunen and D. Lupo, "Reliability of ICA attachment of SMDs on inkjet-printed substrates," Microelectronics Reliability, vol. 52, pp. 2709-2715, 2012.

[22] J. Putaala, J. Hannu, E. Kunnari, M. Mantysalo, O. Nousiainen and H. Jantunen, "Reliability of SMD interconnections on flexible low-temperature substrates with inkjet-printed conductors," Microelectronics Reliability, vol. 54, pp. 272-280, 2013.

[23] J. Liu, "ACA Bonding Technology for Low Cost Electronics Packaging Applications - Current Status and Remaining," in Adhesive Joining and Coating Technology in Electronics Manufacturing, Espoo, Finland, 2000.

[24] J. Liu, "Reliability of surface-mounted anisotropically conductive adhesive joints," Circuit World, vol. 19, no. 4, pp. 4-11, 1993. 
[25] J. Liu and R. Rorgen, "Joining of displays using thermo-setting anisotropically conductive adhesives," Journal of Electronics Manufactuing, vol. 3, 1993.

[26] R. MItsui, S. Takahashi, S.-i. Hakajima, K.-i. Nomura and H. Ushijima, "Study on an Interconnect Technology Toward flexible printed electronics," in Electronics Packaing (ICEP), Toyama, 2014.

[27] D. A. van den Ende and R. C. R. H. L. K. M. C. Rob Hendriks, "Photonic Flash Soldering of Thin Chips and SMD Components on Foils for Flexible Electronics," IEEE Transactions on Components, Packaging, and Manufacturing Technology, vol. 4, no. 11, pp. 1879-1886, 2014.

[28] D. Hackler, D. G. Sime and S. F. Wald, "Enabling Electronics with Physically Flexible ICs and Hybrid Manufacturing," Proceedings of the IEEE, vol. 103, no. 4, pp. 633-643, 2015.

[29] Q. Chu, N. Ghalib and H. Ly, "Investigation of Components Attachment onto Low Temperature Flex Circuit," in FlexTech Alliance for Displays \& Flexible, Printed Electronics, San Jose, 2013.

[30] J. N. Burghartz, W. Appel, C. Harendt, H. Rempp, H. Richter and M. ZImmermann, "Ultra-thin chip technology and applications," Solid-State Electronics, vol. 54, no. 9, pp. 818-829, 2010.

[31] G. Putnik, A. Sluga, H. ElMaraghy, R. Teti, Y. Koren, T. Tolio and B. Hon, "Scalability in manufacturing systems design and operation: State-of-the-art and future developments roadmap," CIRP Annals, Manufacturing Technology, vol. 62, pp. 751-774, 2013.

[32] N. Verma, Y. Hu, L. Huang, W. Rieutort-Louis, J. Robinson, T. Moy, B. Wagner and J. Sturm, "Enabling Scalable Hybrid Systems: Architectures for Exploiting Large-Area Electronics in Applications," Proceedings of the IEEE, vol. 103, no. 4, pp. 690-712, 2015.

[33] Nextflex, "FLEXTECH ALLIANCE RECEIVES \$75 MILLION DEPARTMENT OF DEFENSE AWARD TO CREATE AND MANAGE A FLEXIBLE HYBRID ELECTRONICS MANUFACTURING FACILITY," Nextflex.us, San Jose, 2015.

[34] M. Klas, T. Bauer and U. Tiberi, "Beyond Herding Cats: Aligning Quantitative Technology Evaluation in Large-Scale Research Projects," in Product-Focused Software Process Improvment (PROFES), Berlin, 2013. 


\section{APPENDICES}

Appendix A

\begin{tabular}{|l|c|l|}
\hline \multicolumn{3}{|c|}{ Manufacturing Readiness Level (MRL) } \\
\hline Phase & MRL & State of Development \\
\hline $\begin{array}{l}\text { Phase 3: } \\
\text { Production } \\
\text { Implementation }\end{array}$ & 9 & $\begin{array}{l}\text { Full production process qualified for full } \\
\text { range of parts and full metrics achieved }\end{array}$ \\
\cline { 2 - 3 } & 8 & $\begin{array}{l}\text { Full production process qualified for full } \\
\text { range of parts }\end{array}$ \\
\cline { 2 - 3 } & 7 & Capability and rate confirmed \\
\hline $\begin{array}{l}\text { Phase 2: } \\
\text { Pre production }\end{array}$ & 6 & $\begin{array}{l}\text { Process optimised for production rate } \\
\text { on production equipment }\end{array}$ \\
\cline { 2 - 3 } & 5 & Basic capability demonstrated \\
\hline $\begin{array}{l}\text { Phase 1: } \\
\text { Technology } \\
\text { assessment and } \\
\text { proving }\end{array}$ & 4 & $\begin{array}{l}\text { Production validated in lab environment } \\
\text { Experimental proof of concept } \\
\text { completed }\end{array}$ \\
\hline & 2 & $\begin{array}{l}\text { Application and validity of concept } \\
\text { validated or demonstrated }\end{array}$ \\
\hline
\end{tabular}

Source: “After the TRL,...the MRL.” Rescoll.fr. Blog. 9 January 2014.

Appendix B

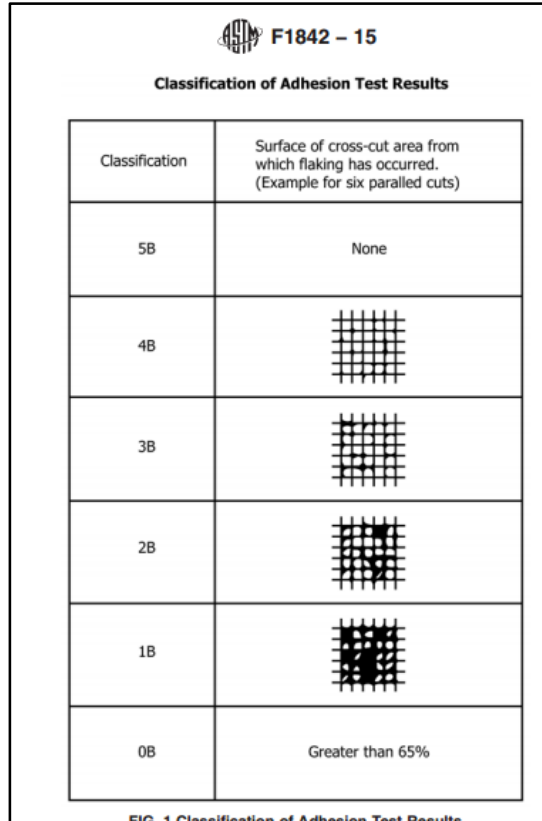

FIG. 1 Classification of Adhesion Test Results

Source: ASTM F1842-15 standards. 